\title{
Distribution of Agricultural NRAs across Countries and Products, 1955-84 and 1985-2007
}

\author{
Kym Anderson \\ kym.anderson@adelaide.edu.au
}

and

Johanna Croser

Johanna.croser@adelaide.edu.au

Agricultural Distortions Working Paper 92, May 2009

This is a product of a research project on Distortions to Agricultural Incentives, under the leadership of Kym Anderson of the World Bank's Development Research Group. The authors are grateful for funding from World Bank Trust Funds provided by the governments of Japan, the Netherlands (BNPP) and the United Kingdom (DfID) and the Australian Research Council. This paper will appear as an Appendix in Political Economy of Distortions to Agricultural Incentives, edited by K. Anderson (forthcoming 2010).

This is part of a Working Paper series (see www.worldbank.org/agdistortions) that is designed to promptly disseminate the findings of work in progress for comment before they are finalized. The views expressed are the authors' alone and not necessarily those of the World Bank and its Executive Directors, nor the countries they represent, nor of the institutions providing funds for this research project. 


\begin{abstract}
The global database developed as an integral part of the World Bank's research project on Distortions to Agricultural Incentives, which is publicly available at www.worldbank.org/agdistortions, provides around 30,000 estimates of nominal rates of assistance to agricultural industries (NRAs) and associated consumer tax equivalents for 75 countries that together account for between 90 and 95 percent of the world's population, farmers, agricultural output and total GDP. They also account for more than 85 percent of farm production and employment in each of Africa, Asia, Latin America and the transition economies of Europe and Central Asia as well as all OECD countries. More than 70 products are included (an average of 11 per country), which represents around 70 percent of the gross value of agricultural production in each of the focus countries, and just under two-thirds of global farm production valued at undistorted prices over the period covered. Not all countries had data for all of the entire 1955-2007 period, but the average number of years covered is 41 per country. This paper provides details of the coverage of the database. It also summarizes the distributions of the NRAs by showing two sets of Box plots for 1955-84 and 1985-2007, one set for various regions of the world, the other for all the covered products for each focus country.
\end{abstract}

Keywords: Agricultural price and trade policies, nominal rates of assistance

JEL codes: F13, F59, H20, N50, O13, Q18

Contact author details:

Kym Anderson

School of Economics

University of Adelaide

Adelaide, SA 5005, Australia

Phone: +61 883034712

kym.anderson@adelaide.edu.au 


\title{
Distribution of Agricultural NRAs across Countries and Products, 1955-84 and 1985-2007
}

\author{
Kym Anderson and \\ Johanna Croser
}

The core global database that was developed as an integral part of the World Bank's research project on Distortions to Agricultural Incentives is publicly available at www.worldbank.org/agdistortions (Anderson and Valenzuela 2008). The case studies from which it is derived cover a total of 75 countries that together account for between 90 and 96 percent of the world's population, farmers, agricultural output and total GDP. They also account for more than 85 percent of farm production and employment in each of Africa, Asia, Latin America and the transition economies of Europe and Central Asia as well as all OECD countries. ${ }^{1}$ The only countries not well represented in the sample are those in the Middle East and the many small ones that together account for less than 5 percent of the global economy.

Nominal rates of assistance and consumer tax equivalents (NRAs and CTEs) are estimated for more than 70 different products, with an average of 11 per country. In aggregate the coverage represents around 70 percent of the gross value of agricultural production in the focus countries, and just under two-thirds of global farm production valued at undistorted prices over the period covered. Not all countries had data for all of the entire 1955-2007 period, but the average number of years covered is 41 per country. Estimates for the transition economies of Europe start in 1992 and for China and Vietnam in 1981 and 1986, respectively. Estimates for two other large Asian countries start later too (India in 1965, Indonesia and Thailand in 1970).

Of the 30 most valuable agricultural products, the NRAs cover 77 percent of global output, ranging from two-thirds for livestock, three-quarters for oilseeds and tropical crops, and five-sixths for grains and tubers. Those products represent an even higher share (85 percent) of global agricultural exports. Tables A.1 to A.5 provide further details of the above

\footnotetext{
${ }^{1}$ A global overview of the results is provided in Anderson (2009), and the detailed country case studies are reported in four regional volumes covering Africa (Anderson and Masters 2009), Asia (Anderson and Martin 2009), Latin American (Anderson and Valdés 2008) and Europe's transition economies (Anderson and Swinnen 2008).
} 
product and country coverage statistics. Figure A.1 shows the number of countries for which NRA and CTE estimates are produced for each of 30 key farm products, while Figure A.2 shows the share of global production of each of those 30 key farm products that is covered by NRA and CTE estimates.

This book is focused primarily on explaining the differences across countries, products and time of distortions to farmers' incentives, particularly as summarized by nominal rates of assistance. Figure A.3 summarizes the distributions and shows, for various regions of the world, a set of Box plots. The long bar in those plots shows the range within which 95 percent of the NRAs fall: 50 percent fall in the shaded area, and the vertical line within the shaded area is the median NRA for the sample time period. Then in Figure A.4 we provide similar Box plots of all the covered products for each focus country. ${ }^{2}$ In both figures the sub-periods before and from 1985 are shown separately. A break in the mid-1980s roughly coincides with when many developing countries and some high-income countries began to reform their agricultural and trade policies. Also, the earlier period coincides with that covered by the seminal World Bank study of 18 developing countries by Krueger, Schiff and Valdés (1991), and the later period coincides with the time series of estimates of agricultural distortions in high-income countries by the OECD (2008).

\section{References}

Anderson K. (ed.) (2009), Distortions to Agricultural Incentives: A Global Perspective, 1955 to 2007, London: Palgrave Macmillan and Washington DC: World Bank.

Anderson, K. and W. Martin (eds.) (2009), Distortions to Agricultural Incentives in Asia, Washington DC: World Bank.

Anderson, K. and W. Masters (eds.) (2009), Distortions to Agricultural Incentives in Africa, Washington DC: World Bank.

Anderson, K. and J. Swinnen (eds.) (2008), Distortions to Agricultural Incentives in Europe's Transition Economies, Washington DC: World Bank.

Anderson, K. and A. Valdés (eds.) (2008), Distortions to Agricultural Incentives in Latin America, Washington DC: World Bank.

\footnotetext{
${ }^{2}$ We are grateful to Kishore Gawande for suggesting this type of plot be used to summarize the data.
} 
Anderson, K. and E. Valenzuela (2008), “Estimates of Global Distortions to Agricultural Incentives, 1955 to 2007”, World Bank, Washington DC, available on the Database page at www.worldbank.org/agdistortions.

Krueger, A.O., M. Schiff and A. Valdés (1991), The Political Economy of Agricultural Pricing Policy, Volume 1: Latin America, Volume 2: Asia, and Volume 3: Africa and the Mediterranean, Baltimore: Johns Hopkins University Press for the World Bank.

OECD (2008 and earlier years), Agricultural Policies in OECD Countries: Monitoring and Evaluation, Paris: Organization for Economic Cooperation and Development. 
Table A.1: Summary of NRA coverage statistics, World Bank agricultural distortions project

Number and size of countries

Africa

Asia

Latin America

SUB-TOTAL, all DCs

European transition econs

High-income countries

TOTAL

Number of years covered

Africa

Asia

Latin America

SUB-TOTAL, all DCs

European transition econs

High-income countries

TOTAL

Number of products covered

Africa

Asia

Latin America

SUB-TOTAL, all DCs

European transition econs

High-income countries

TOTAL
Number

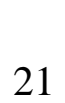

12

$\underline{8}$

41

14

$\frac{20}{75}$

Maximum

51

53

51

53

47

53

51

Maximum

44

35

27

59

25

39

74
2000-04 \% of global:

\begin{tabular}{rr}
\hline Popn. & Ag GDP \\
\hline 11 & 7 \\
51 & 37 \\
$\underline{7}$ & $\underline{8}$ \\
$\mathbf{6 9}$ & $\mathbf{5 2}$ \\
7 & 7 \\
$\underline{14}$ & $\underline{33}$ \\
$\mathbf{9 2}$ & $\mathbf{9 2}$
\end{tabular}

Av. per country

43

42

39

43

17

52

41

Av. per country

\section{8}

8

10

9

12

15

11

Total number of NRA ests. (years and products)

Total

7318

3546

2881

13745

2847

13377

29969
Av. per country

348

296

360

335

203

669

400

Share of global agric. prod'n of $30 \quad \%$

key covered products, 2000-04

$\begin{array}{lr}\text { Africa } & 5 \\ \text { Asia } & 28 \\ \text { Latin America } & 7 \\ \text { SUB-TOTAL, focus DCs } & 41 \\ \text { European transition econs } & 6 \\ \text { High-income countries } & 29 \\ \text { TOTAL, focus countries } & 77\end{array}$

Source: Calculated from data in Anderson and Valenzuela (2008) 
Table A.2: Coverage of gross value of global agricultural production at undistorted prices, for 30 key products and four product groups, 2000-04

\begin{tabular}{|c|c|c|}
\hline & $\begin{array}{r}\text { Coverage of } \\
\text { product's } \\
\text { global } \\
\text { GVOP, \% }\end{array}$ & $\begin{array}{r}\text { Product's } \\
\text { share of global } \\
\text { GVOP of } 30 \\
\text { key } \\
\text { products, \% }\end{array}$ \\
\hline Grains and tubers & 85 & 31.4 \\
\hline Rice & 92 & 9.5 \\
\hline Wheat & 89 & 8.0 \\
\hline Maize & 94 & 7.2 \\
\hline Cassava & 41 & 2.4 \\
\hline Barley & 83 & 1.6 \\
\hline Sorghum & 70 & 0.7 \\
\hline Yam & 77 & 0.8 \\
\hline Millet & 29 & 0.6 \\
\hline Oat & 61 & 0.3 \\
\hline Oilseeds & 78 & 8.1 \\
\hline Soybean & 96 & 4.0 \\
\hline Groundnut & 34 & 1.2 \\
\hline Palmoil & 90 & 1.0 \\
\hline Rapeseed & 64 & 1.0 \\
\hline Sunflower & 77 & 0.6 \\
\hline Sesame & 9 & 0.3 \\
\hline Tropical crops & 74 & 7.6 \\
\hline Sugar & 87 & 2.5 \\
\hline Cotton & 88 & 1.9 \\
\hline Coconut & 60 & 0.9 \\
\hline Coffee & 75 & 0.7 \\
\hline Rubber & 74 & 0.7 \\
\hline Tea & 21 & 0.6 \\
\hline Cocoa & 71 & 0.4 \\
\hline Chickpea & 61 & 0.3 \\
\hline Livestock products & 72 & 52.9 \\
\hline Pigmeat & 91 & 12.4 \\
\hline Milk & 83 & 12.0 \\
\hline Beef & 69 & 10.5 \\
\hline Poultry & 81 & 7.6 \\
\hline Egg & 35 & 6.5 \\
\hline Sheepmeat & 27 & 3.3 \\
\hline Wool & 42 & 0.6 \\
\hline All above products & 77 & 100.0 \\
\hline
\end{tabular}

Note: Fruit and vegetables, which account for around 23 percent of global ag GVOP in 2001 (according to GTAP database - see Dimaranan 2006) are not included in this table, even though several fruits and vegetables for selected countries are included in the analysis.

Source: Calculated from data in Anderson and Valenzuela (2008). 
Table A.3: Current project's coverage of national agricultural production in focus countries at undistorted prices, regional averages, 1980 to 2004

(percent)

$\begin{array}{llll}\text { Africa } & 67 & 66 & 68 \\ \text { Asia } & 75 & 73 & 66 \\ \text { Latin America } & 65 & 69 & 70 \\ \text { SUB-TOTAL, focus DCs } & 72 & 72 & 67 \\ \text { European transition econs } & 62 & 61 & 63 \\ \text { High-income countries } & 73 & 73 & 72 \\ \text { TOTAL, all focus countries } & \mathbf{7 2} & \mathbf{7 1} & \mathbf{6 8}\end{array}$

Source: Calculated from data in Anderson and Valenzuela (2008) 
Table A.4: Derived shares of global agricultural production for 30 major covered products, Africa, Asia, Latin America, Europe's transition economies and high-income regions, 200004

\begin{tabular}{|c|c|c|c|c|c|c|c|c|c|}
\hline & \multicolumn{8}{|c|}{ Regional shares (\%) of global gross value of agric production (GVOP) } & \multirow{3}{*}{$\begin{array}{r}\text { Product share } \\
\text { (\%) of global } \\
\text { GVOP of } 30 \\
\text { products }\end{array}$} \\
\hline & \multicolumn{6}{|c|}{ Covered products in focus countries } & \multirow[t]{2}{*}{ Residual } & \multirow[t]{2}{*}{ World } & \\
\hline & Africa & Asia & LAC & ECA & HIC & All & & & \\
\hline Grains+tubers & 11 & 40 & 5 & 6 & 23 & 85 & 15 & 100 & 31 \\
\hline Rice & 3 & 81 & 2 & 0 & 5 & 92 & 8 & 100 & 9 \\
\hline Wheat & 6 & 32 & 4 & 14 & 33 & 89 & 11 & 100 & 8 \\
\hline Maize & 11 & 26 & 13 & 5 & 40 & 94 & 6 & 100 & 7 \\
\hline Cassava & 39 & 2 & 0 & 0 & 0 & 41 & 59 & 100 & 2 \\
\hline Barley & 0 & 0 & 1 & 25 & 57 & 83 & 17 & 100 & 2 \\
\hline Sorghum & 26 & 13 & 14 & 0 & 17 & 70 & 30 & 100 & 1 \\
\hline Yam & 77 & 0 & 0 & 0 & 0 & 77 & 23 & 100 & 1 \\
\hline Millet & 29 & 0 & 0 & 0 & 0 & 29 & 71 & 100 & 1 \\
\hline Oat & 0 & 0 & 0 & 28 & 33 & 61 & 39 & 100 & 0 \\
\hline Oilseeds & 4 & 22 & 20 & 4 & 28 & 78 & 22 & 100 & 8 \\
\hline Soybean & 0 & 15 & 37 & 0 & 43 & 96 & 4 & 100 & 4 \\
\hline Groundnut & 17 & 17 & 0 & 0 & 0 & 34 & 66 & 100 & 1 \\
\hline Palmoil & 8 & 81 & 2 & 0 & 0 & 90 & 10 & 100 & 1 \\
\hline Rapeseed & 0 & 12 & 0 & 4 & 47 & 64 & 36 & 100 & 1 \\
\hline Sunflower & 3 & 5 & 16 & 39 & 14 & 77 & 23 & 100 & 1 \\
\hline Sesame & 8 & 0 & 0 & 0 & 0 & 9 & 91 & 100 & 0 \\
\hline $\begin{array}{l}\text { Tropical } \\
\text { crops }\end{array}$ & 10 & 36 & 12 & 5 & 11 & 74 & 26 & 100 & 8 \\
\hline Sugar & 5 & 43 & 17 & 6 & 16 & 87 & 13 & 100 & 2 \\
\hline Cotton & 17 & 30 & 5 & 14 & 22 & 88 & 12 & 100 & 2 \\
\hline Coconut & 0 & 60 & 0 & 0 & 0 & 60 & 40 & 100 & 1 \\
\hline Coffee & 11 & 12 & 52 & 0 & 0 & 75 & 25 & 100 & 1 \\
\hline Rubber & 0 & 74 & 0 & 0 & 0 & 74 & 26 & 100 & 1 \\
\hline Tea & 11 & 10 & 0 & 0 & 0 & 21 & 79 & 100 & 1 \\
\hline Cocoa & 68 & 0 & 3 & 0 & 0 & 71 & 29 & 100 & 0 \\
\hline Chickpea & 0 & 61 & 0 & 0 & 0 & 61 & 39 & 100 & 0 \\
\hline $\begin{array}{l}\text { Livestock } \\
\text { products }\end{array}$ & 3 & 21 & 6 & 7 & 36 & 72 & 28 & 100 & 53 \\
\hline Pigmeat & 0 & 49 & 3 & 6 & 34 & 91 & 9 & 100 & 12 \\
\hline Milk & 3 & 21 & 4 & 12 & 43 & 83 & 17 & 100 & 12 \\
\hline Beef & 6 & 1 & 16 & 5 & 41 & 69 & 31 & 100 & 10 \\
\hline Poultry & 2 & 27 & 9 & 5 & 38 & 81 & 19 & 100 & 8 \\
\hline Egg & 0 & 1 & 3 & 7 & 23 & 35 & 65 & 100 & 6 \\
\hline Sheepmeat & 5 & 0 & 0 & 3 & 18 & 27 & 73 & 100 & 3 \\
\hline Wool & 0 & 0 & 0 & 0 & 42 & 42 & 58 & 100 & 1 \\
\hline $\begin{array}{l}\text { Total of above } \\
\mathbf{3 0} \text { products }\end{array}$ & 6 & 28 & 7 & 6 & 29 & 77 & 23 & 100 & 100 \\
\hline
\end{tabular}

Sources: Authors' calculations based on FAO commodity balance, to get volume of production data, and the Agricultural Distortions database to convert this to value of production at undistorted prices so we can add up across commodities. 
Table A.5: Share of regional agricultural production for 30 major covered products, Africa, Asia, Latin America, Europe's transition economies and high-income regions, 2000-04

Covered product shares (\%) of regional gross value of agricultural production of focus countries

\begin{tabular}{|c|c|c|c|c|c|c|}
\hline & \\
\hline & Africa & Asia & LAC & ECA & HIC & All \\
\hline Grains + tubers & 37.3 & 21.8 & 14.3 & 16.8 & 15.2 & 19.6 \\
\hline Rice & 2.8 & 13.6 & 1.9 & 0.1 & 1.0 & 6.3 \\
\hline Wheat & 4.7 & 4.6 & 3.0 & 10.2 & 5.6 & 5.3 \\
\hline Maize & 8.4 & 3.3 & 8.3 & 2.7 & 6.2 & 5.0 \\
\hline Cassava & 9.8 & 0.1 & 0.0 & na & na & 0.7 \\
\hline Barley & na & 0.0 & 0.2 & 3.2 & 2.0 & 1.0 \\
\hline Sorghum & 2.5 & 0.2 & 0.9 & na & 0.3 & 0.4 \\
\hline Yam & 7.0 & na & na & na & na & 0.5 \\
\hline Millet & 2.1 & na & na & na & na & 0.1 \\
\hline Oat & na & na & na & 0.6 & 0.2 & 0.1 \\
\hline Oilseeds & 3.3 & 3.1 & 14.5 & 2.2 & 4.8 & 4.5 \\
\hline Soybean & 0.0 & 1.1 & 13.3 & 0.0 & 3.6 & 2.8 \\
\hline Groundnut & 2.0 & 0.4 & 0.0 & na & na & 0.3 \\
\hline Palmoil & 0.9 & 1.4 & 0.1 & na & na & 0.6 \\
\hline Rapeseed & na & 0.2 & na & 0.2 & 1.0 & 0.4 \\
\hline Sunflower & 0.2 & 0.1 & 0.9 & 2.0 & 0.2 & 0.4 \\
\hline Sesame & 0.2 & na & 0.0 & na & na & 0.0 \\
\hline Tropical crops & 7.9 & 5.1 & 8.1 & 3.5 & 1.7 & 4.2 \\
\hline Sugar & 1.2 & 1.9 & 3.8 & 1.3 & 0.8 & 1.6 \\
\hline Cotton & 2.3 & 1.0 & 0.9 & 2.1 & 0.9 & 1.1 \\
\hline Coconut & na & 0.8 & na & na & na & 0.3 \\
\hline Coffee & 0.8 & 0.1 & 3.3 & na & na & 0.4 \\
\hline Rubber & na & 0.9 & na & na & na & 0.4 \\
\hline Tea & 0.7 & 0.1 & na & na & na & 0.1 \\
\hline Cocoa & 3.0 & 0.0 & 0.1 & na & na & 0.2 \\
\hline Chickpea & na & 0.3 & na & na & na & 0.1 \\
\hline $\begin{array}{l}\text { Livestock } \\
\text { products }\end{array}$ & 13.7 & 19.0 & 29.9 & 30.5 & 40.4 & 27.9 \\
\hline Pigmeat & na & 10.6 & 3.0 & 6.6 & 8.9 & 8.3 \\
\hline Milk & 3.5 & 4.5 & 4.4 & 11.8 & 11.1 & 7.3 \\
\hline Beef & 6.8 & 0.2 & 14.7 & 4.6 & 9.1 & 5.3 \\
\hline Poultry & 1.6 & 3.6 & 5.9 & 2.9 & 6.2 & 4.5 \\
\hline Egg & na & 0.1 & 1.9 & 3.7 & 3.2 & 1.7 \\
\hline Sheepmeat & 1.8 & na & na & 0.9 & 1.3 & 0.7 \\
\hline $\begin{array}{l}\text { Wool } \\
\text { Sol }\end{array}$ & na & na & na & na & 0.6 & 0.2 \\
\hline 30 & 62.2 & 48.8 & 66.7 & 52.9 & 62.2 & 56.2 \\
\hline All covered & 68.2 & 66.1 & 70.3 & 60.9 & 72.4 & 68.3 \\
\hline Non-covered & 31.8 & 33.9 & 29.7 & 39.1 & 27.6 & 31.7 \\
\hline All agric & 100.0 & 100.0 & 100.0 & 100.0 & 100.0 & 100.0 \\
\hline
\end{tabular}

Source: Calculated from data in Anderson and Valenzuela (2008). 
Figure A.1: Number of countries for which NRA and CTE estimates are provided for 30 key farm products

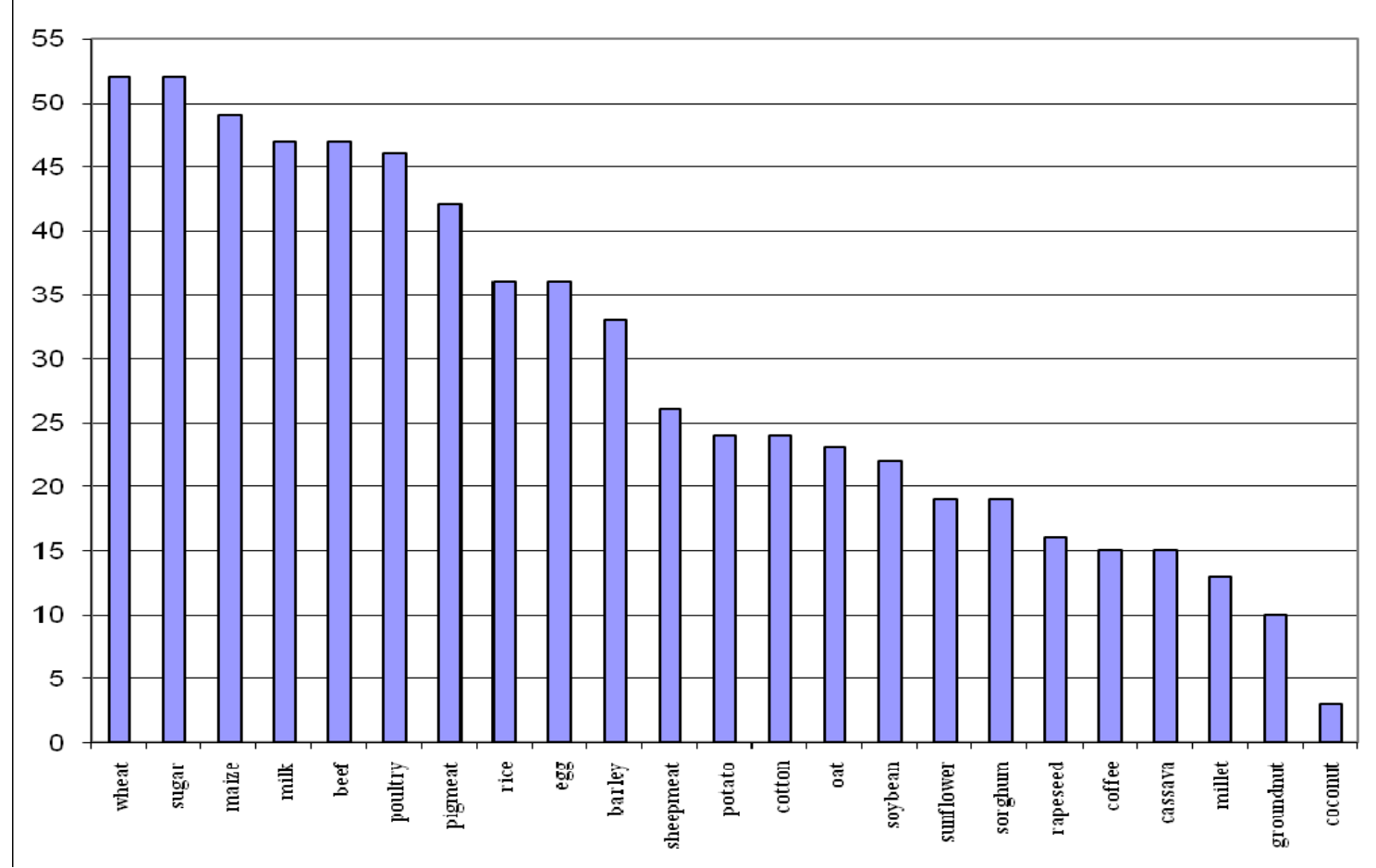

Source: Drawn from estimates in Anderson and Valenzuela (2008). 
Figure A.2: Shares of global production of 30 key farm products covered in NRA and CTE estimates

$$
\text { (percent) }
$$

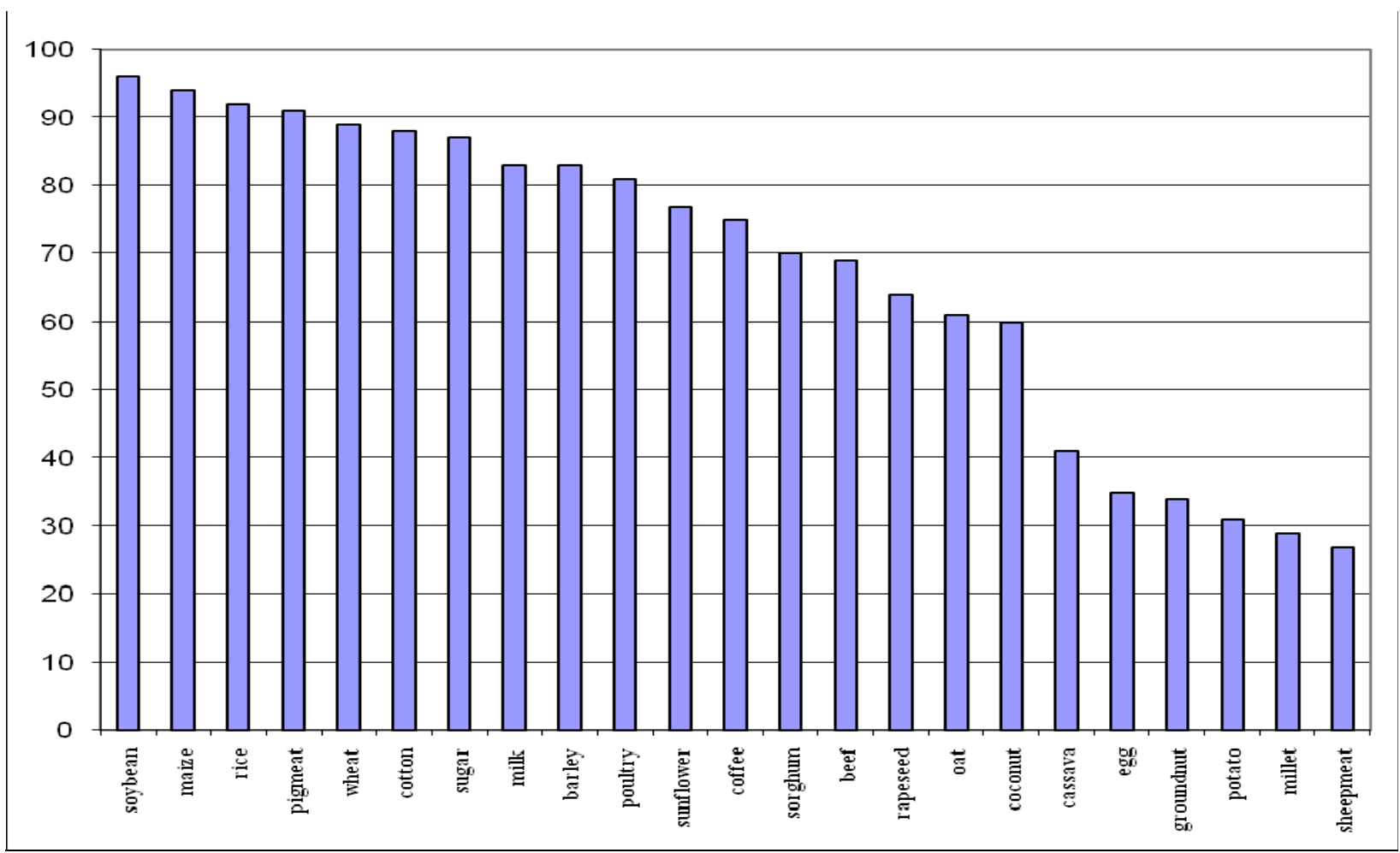

Source: Drawn from estimates in Anderson and Valenzuela (2008). 
Figure A.3: Box plot distributions of NRAs for 25 major agricultural products, various regions of the world, 1955 to 2007

(long bar shows range within which 95 percent of the NRAs fall: 50 percent fall in the shaded area, and the vertical line within the shaded area is the median NRA for the sample period)

(a) All 21 focus African countries, plus Turkey $(\mathrm{n}=7988)$
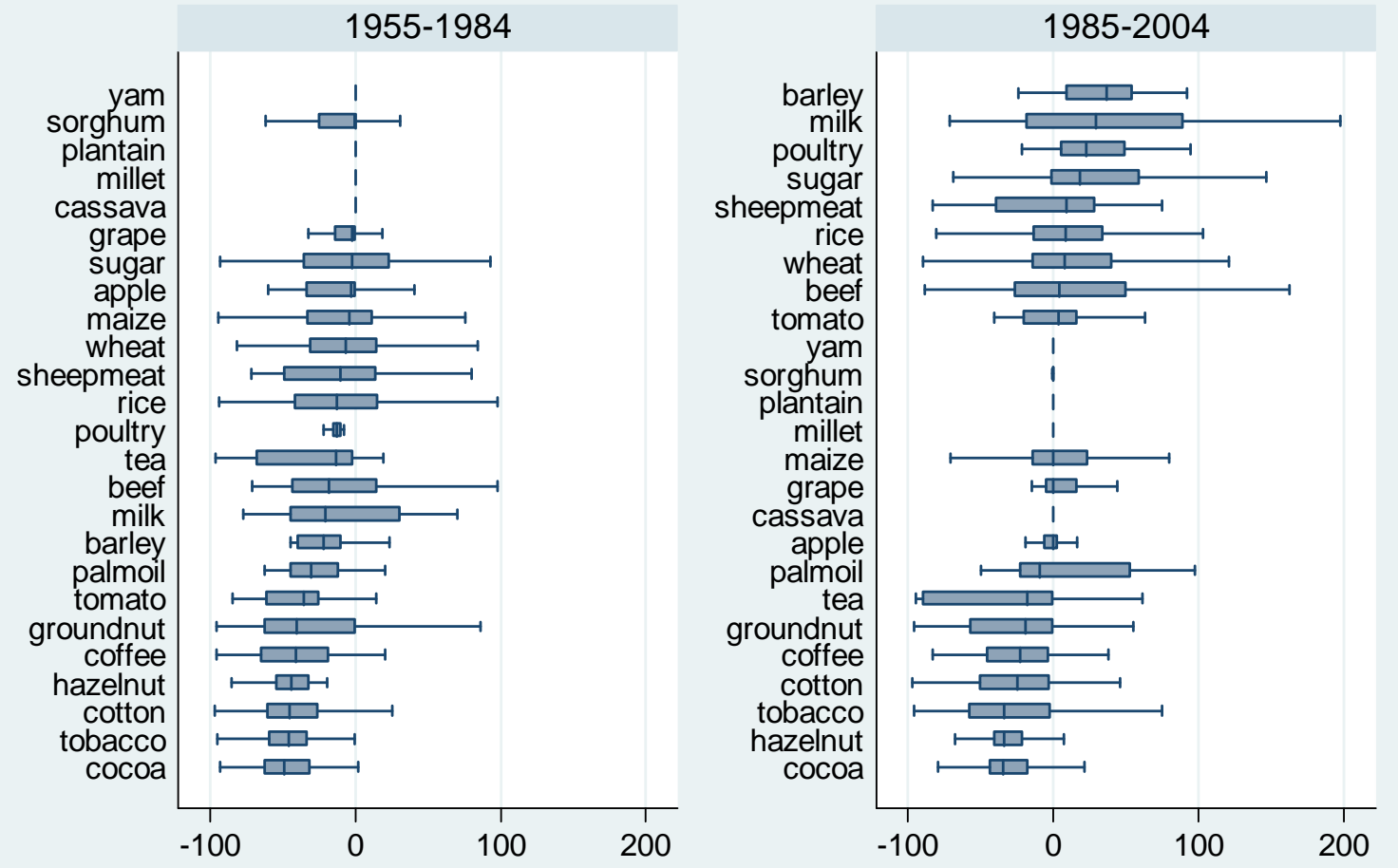

(b) All 12 focus Asian developing economies (excluding Japan) $(\mathrm{n}=5410)$
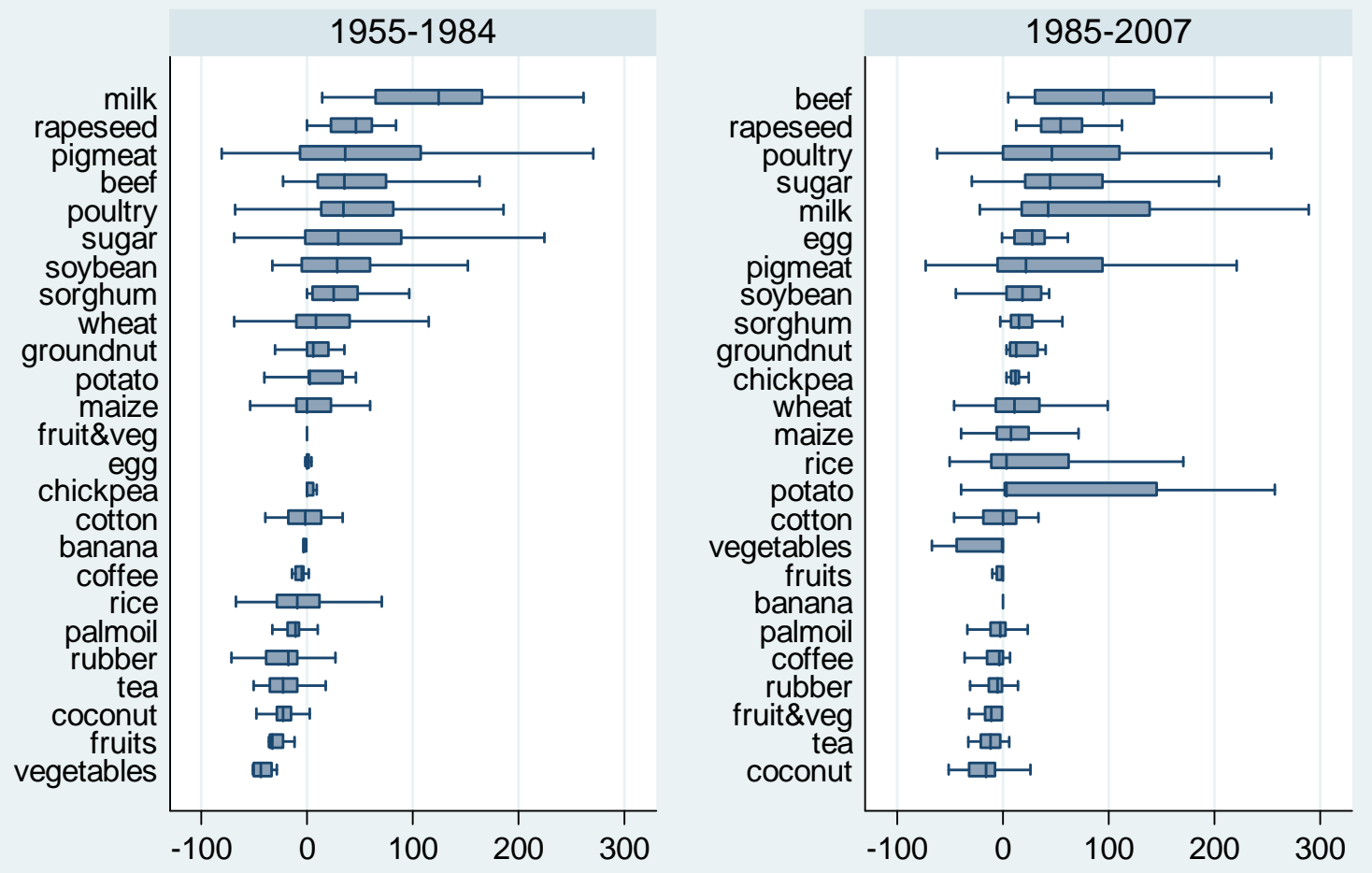
Figure A.3 (continued): Box plot distributions of NRAs for 25 major agricultural products, various regions of the world, 1955 to 2007

(c) All 8 focus Latin American countries $(n=4180)$
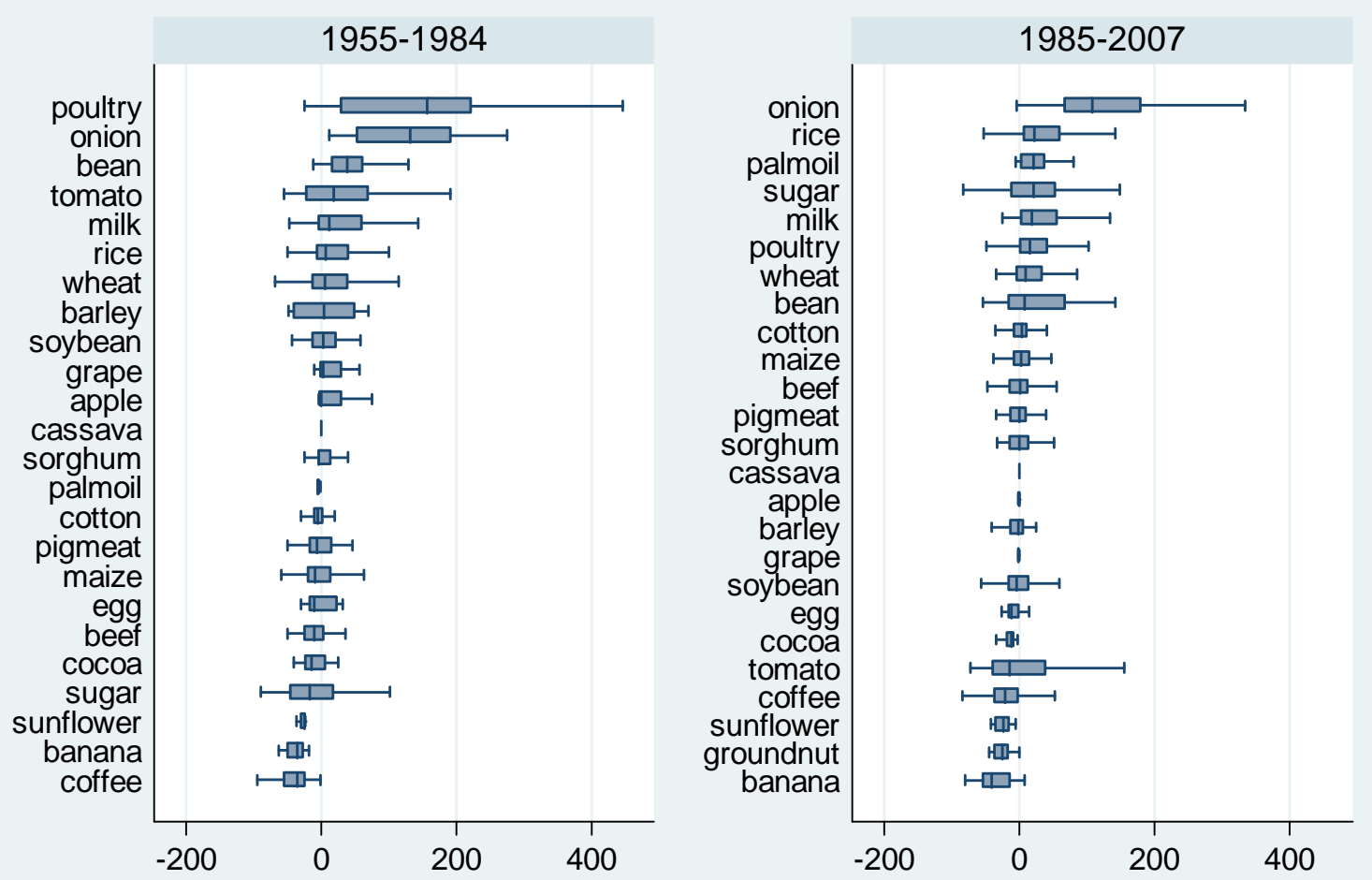

(d) All 12 focus European transition economies (excluding Turkey) $(\mathrm{n}=2398)$
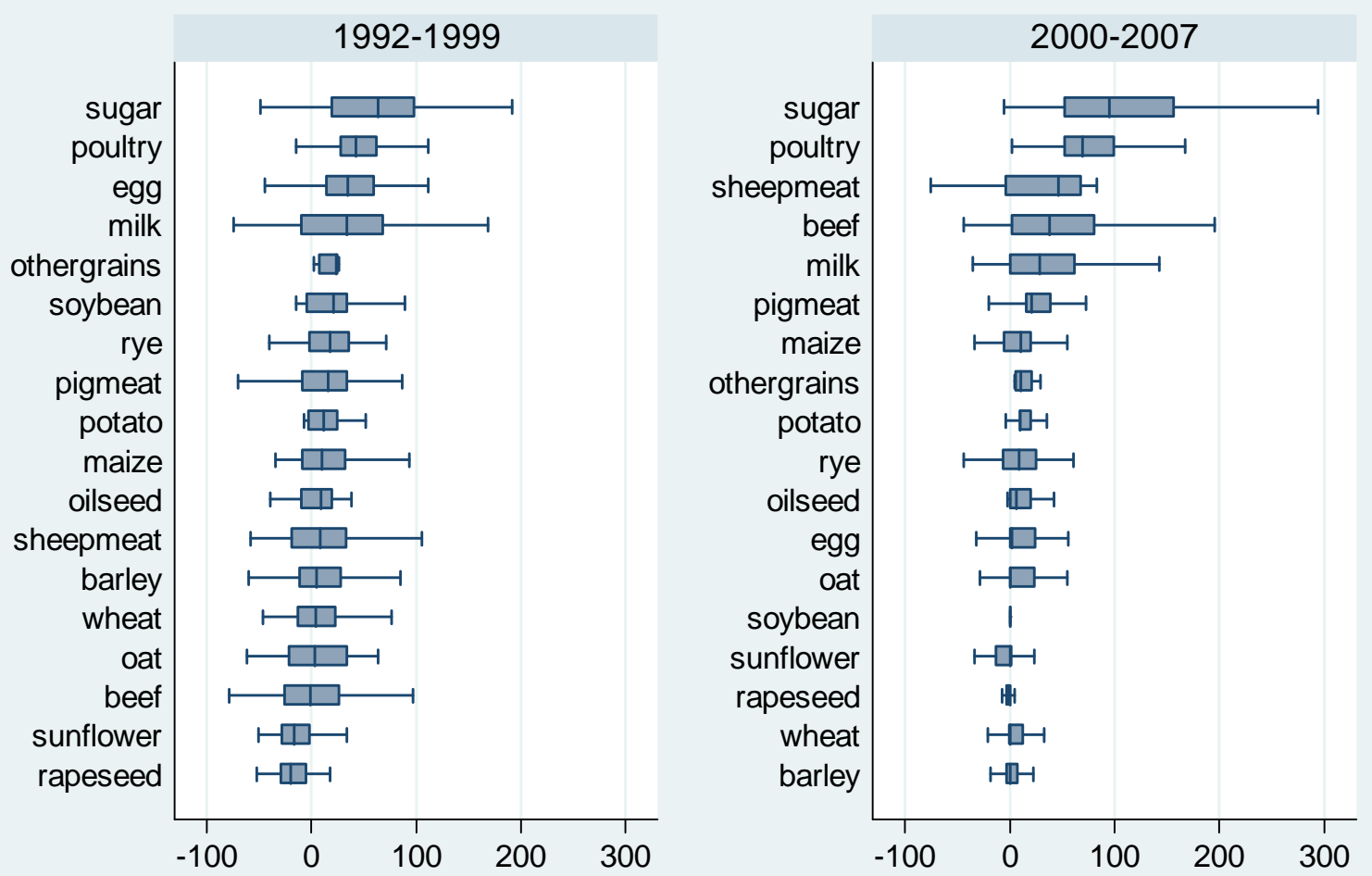
Figure A.3 (continued): Box plot distributions of NRAs for 25 major agricultural products, various regions of the world, 1955 to 2007

(e) All 41 focus developing economies (including Turkey) $(\mathrm{n}=14392)$
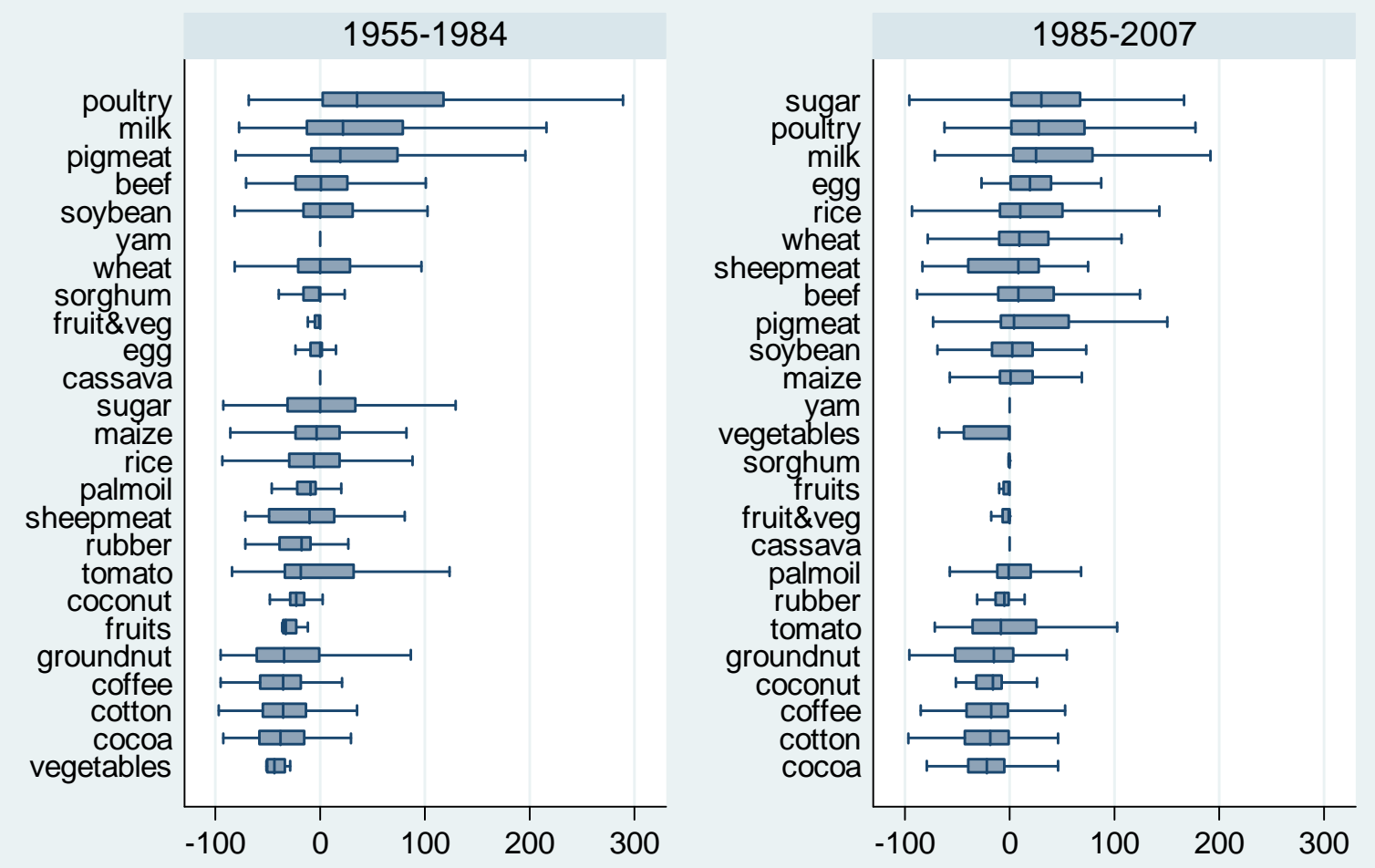

(f) All 20 focus high-income countries $(n=12970)$

1955-1984

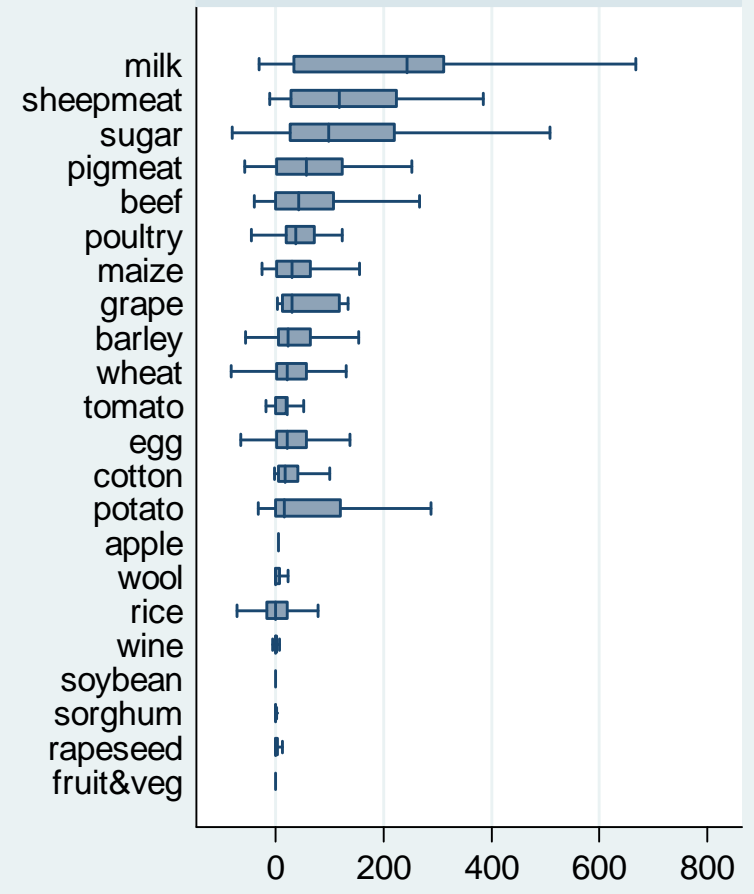

1985-2007

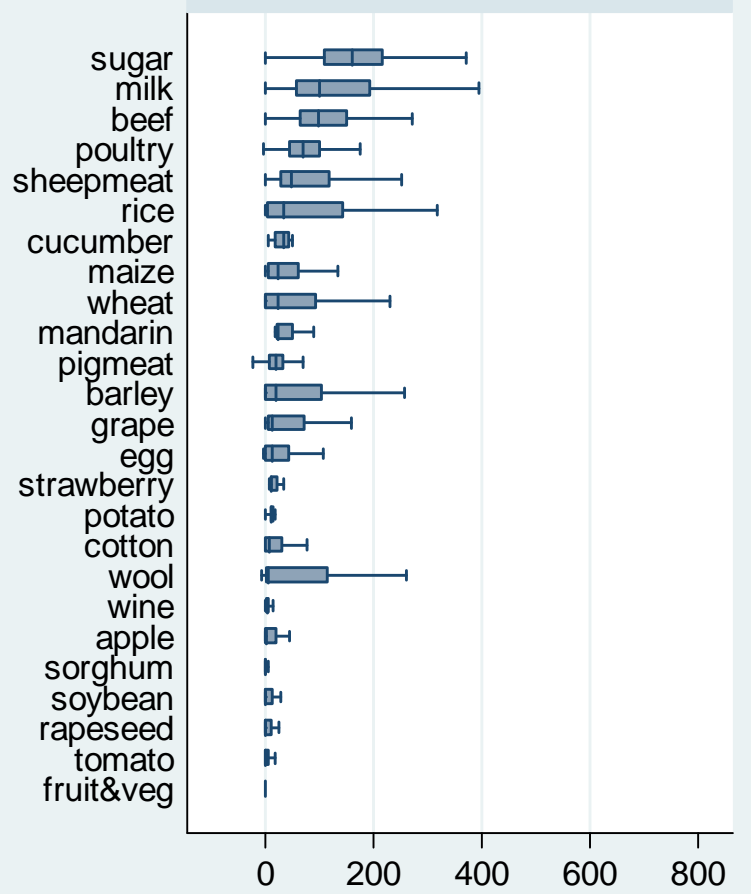


Figure A.3 (continued): Box plot distributions of NRAs for 25 major agricultural products, various regions of the world, 1955 to 2007

(g) All 73 focus economies of the world ( $\mathrm{n}=34833$ )
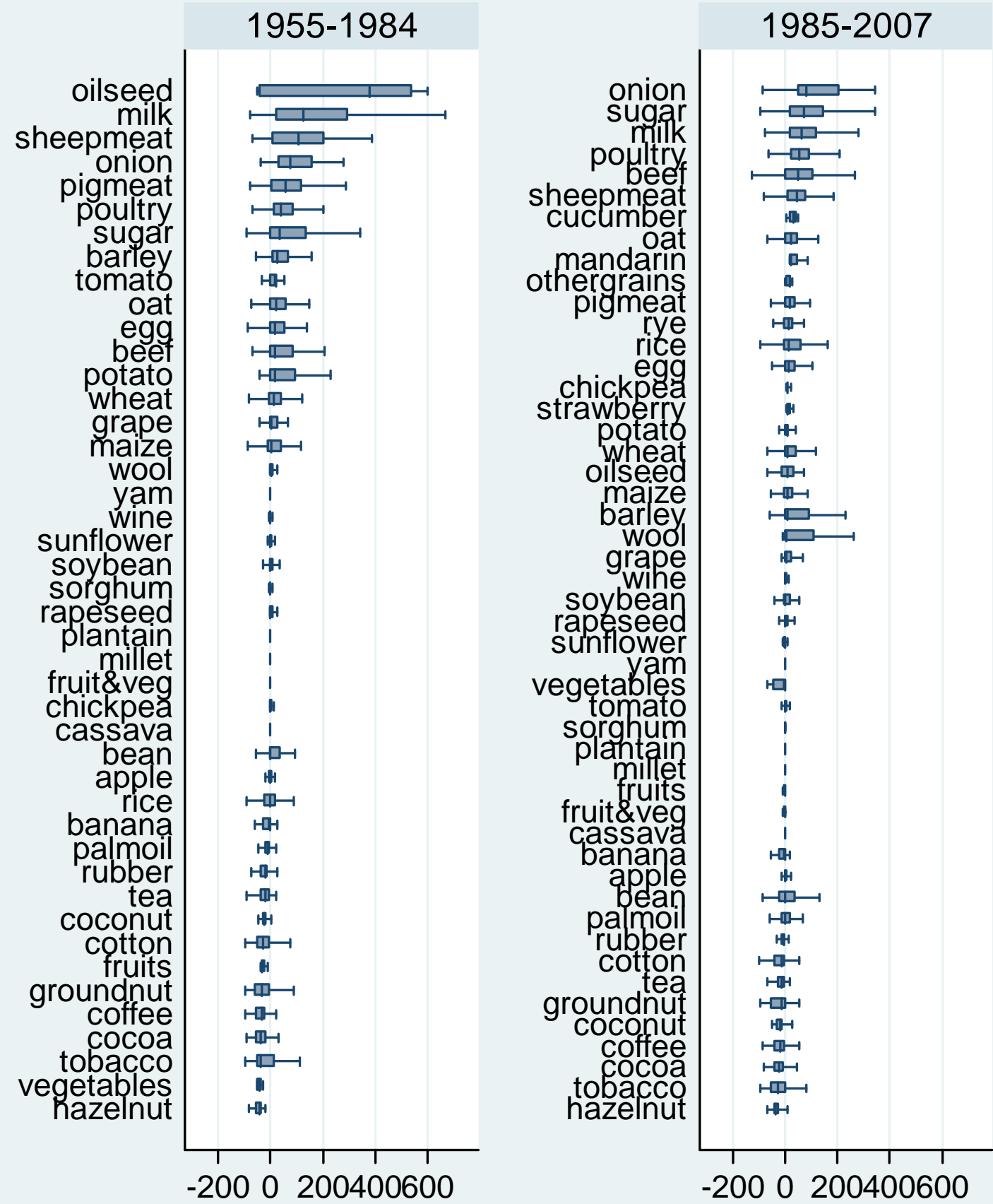

Source: Drawn from estimates in Anderson and Valenzuela (2008). 
Figure A.4: Box plot distributions of NRAs of all covered agricultural products for each focus economy, 1955 to 2007 (long bar shows range within which 95 percent of the NRAs fall: the shaded area has 50 percent of NRAs, and the vertical line within the shaded area is the median NRA for the available sample period)

(a) 16 African countries (excluding 5 cotton-only countries) and Turkey

Cameroon $(\mathrm{n}=510)$
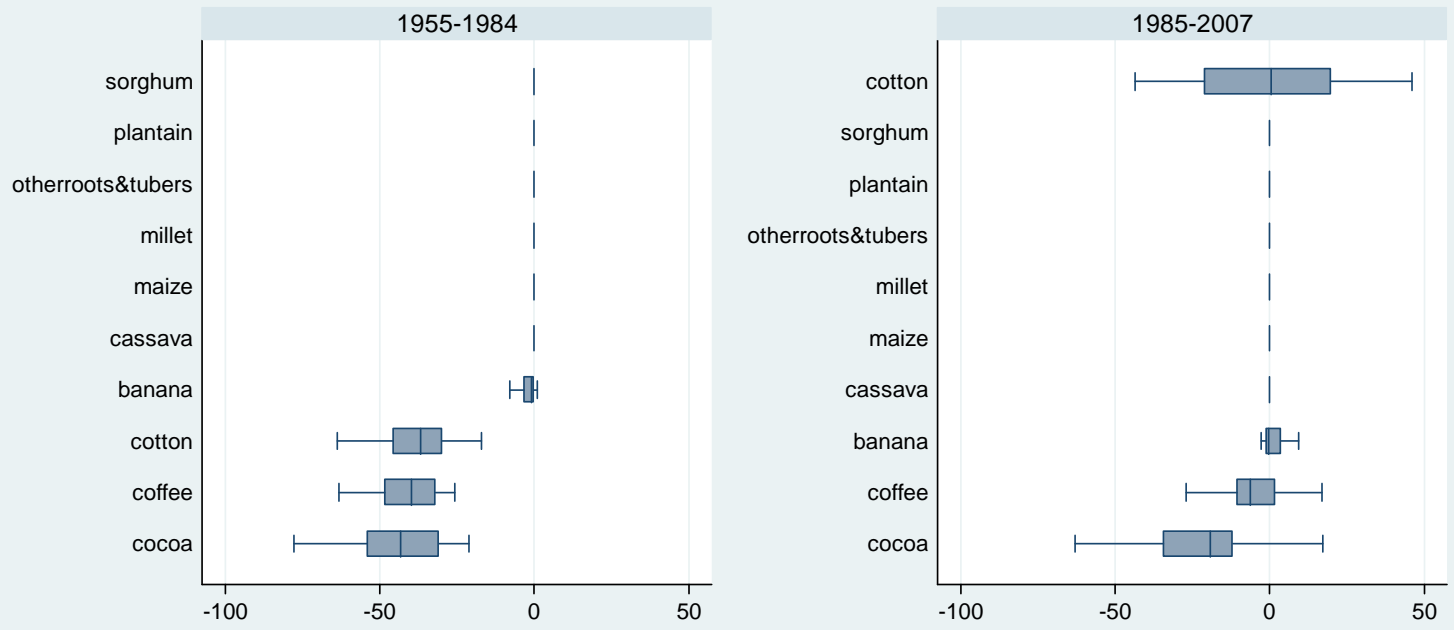

Cote d'Ivoire $(n=357)$
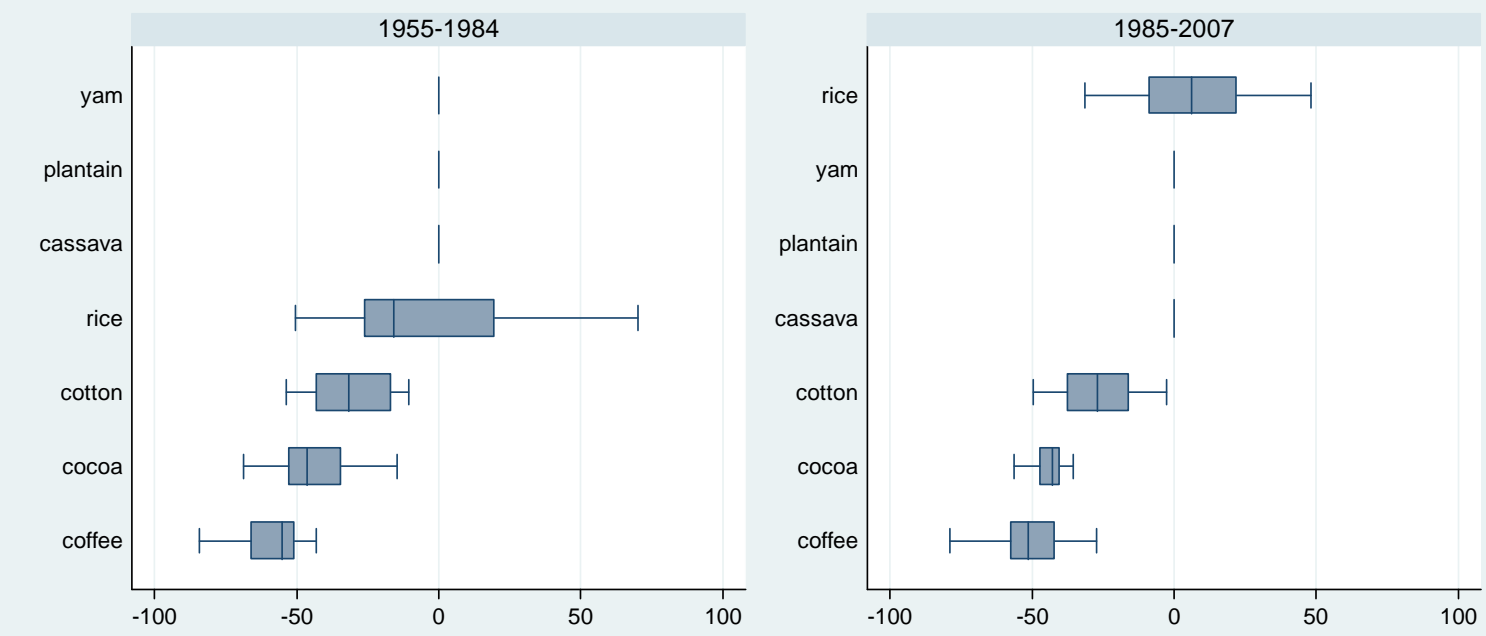
Egypt $(\mathrm{n}=357)$
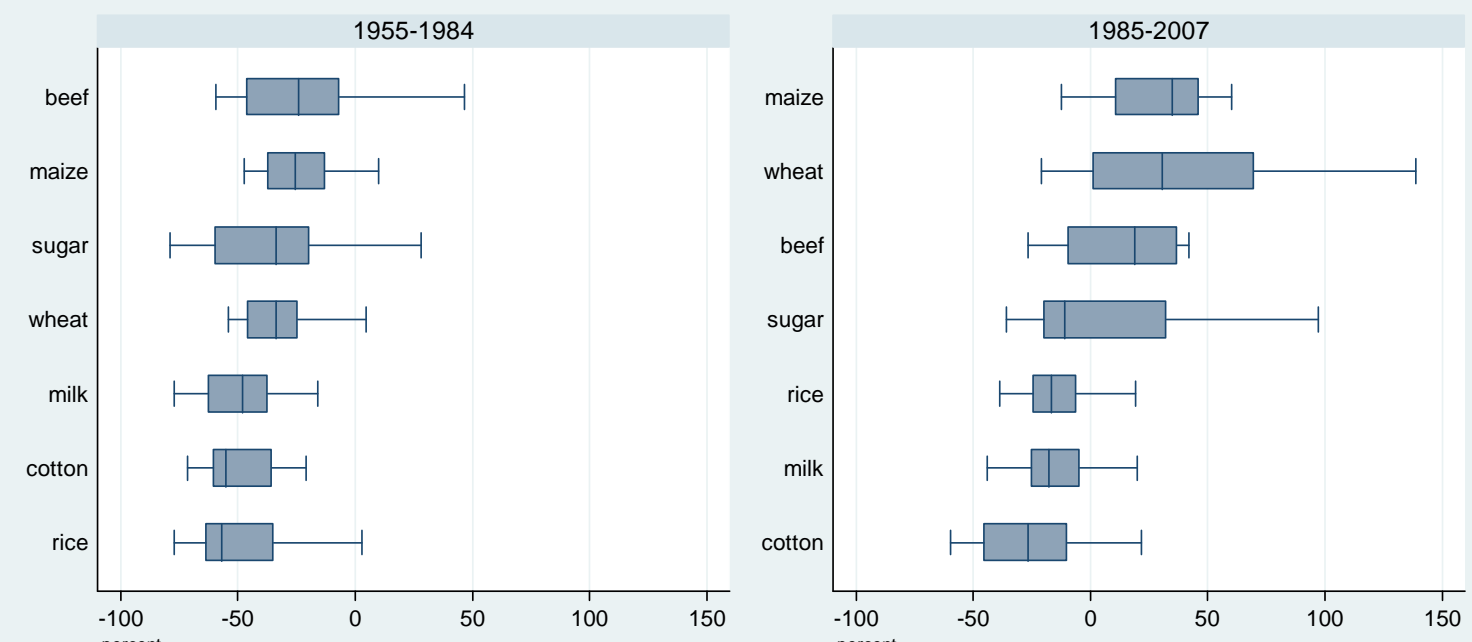

Ethiopia $(\mathrm{n}=408)$
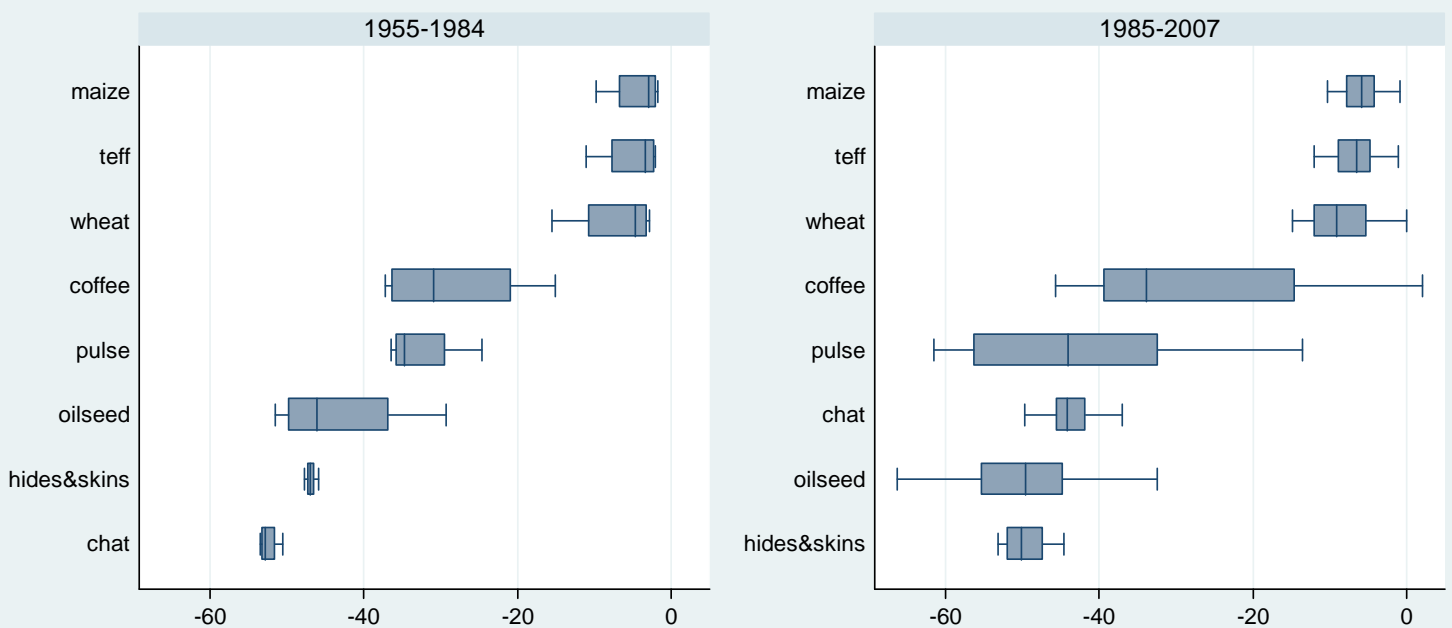

Ghana $(\mathrm{n}=357)$
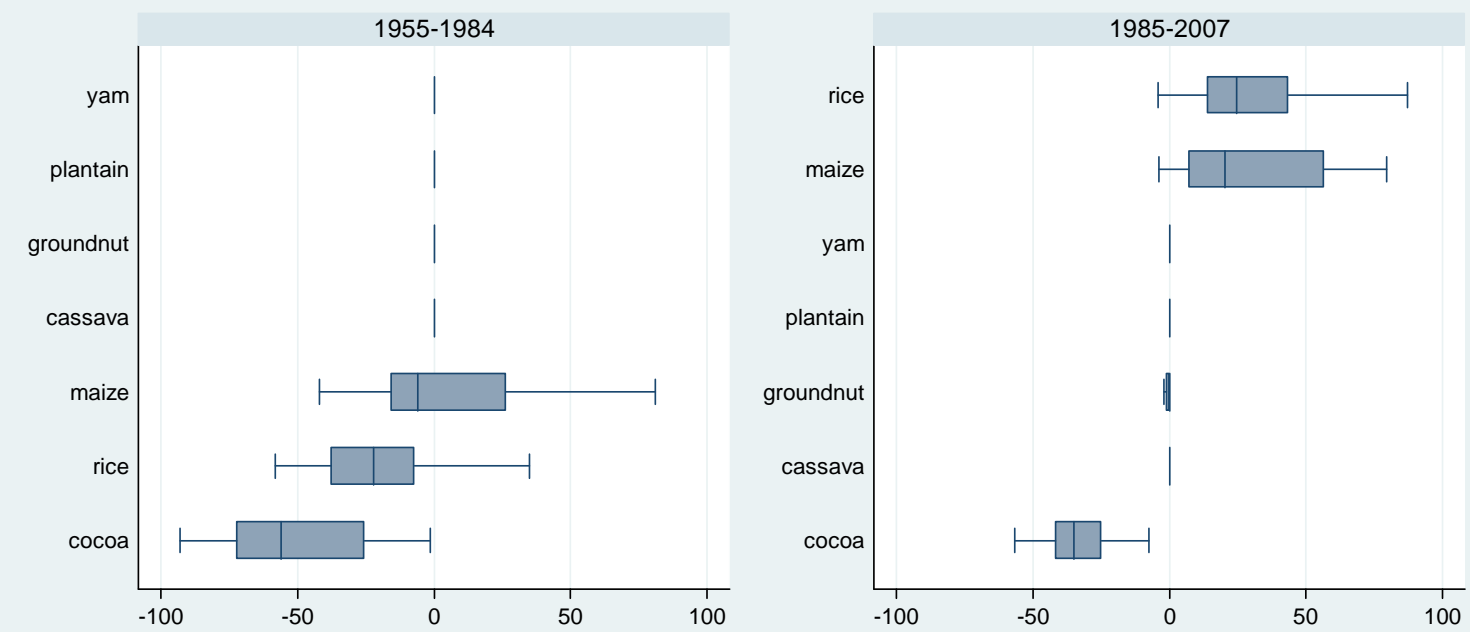
Kenya $(\mathrm{n}=343)$
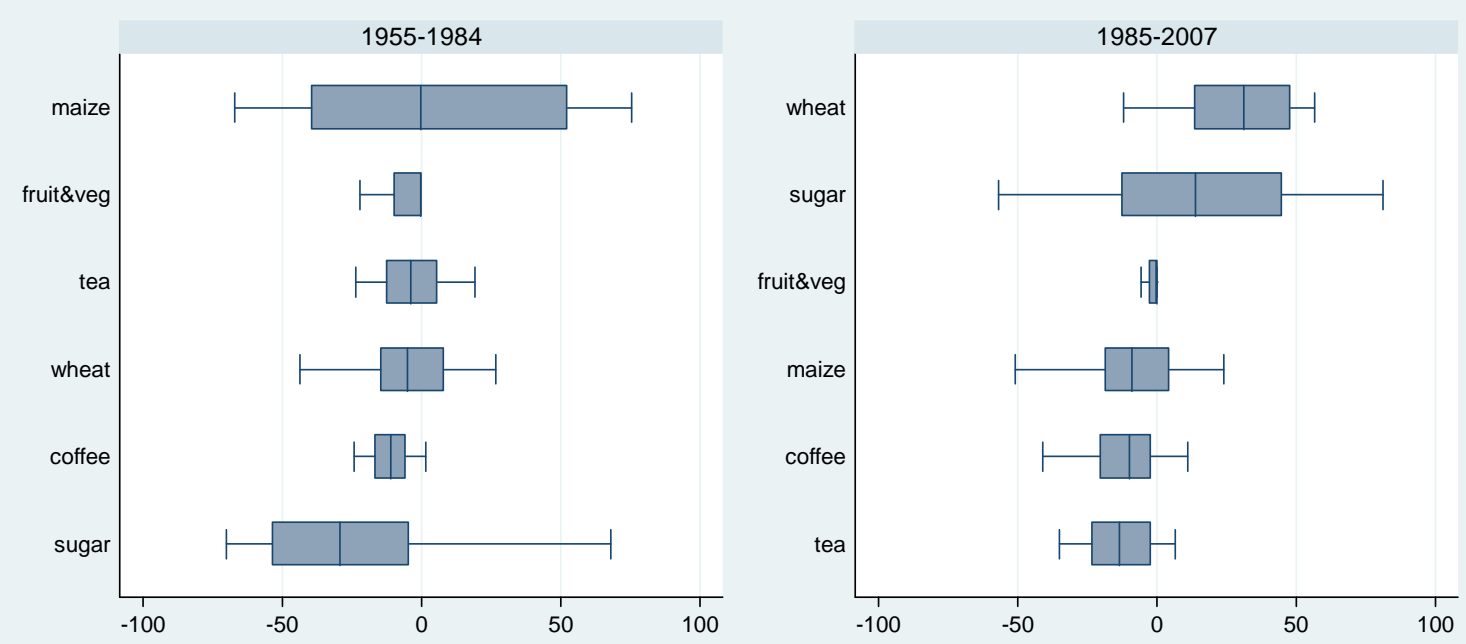

Madagascar $(\mathrm{n}=510)$
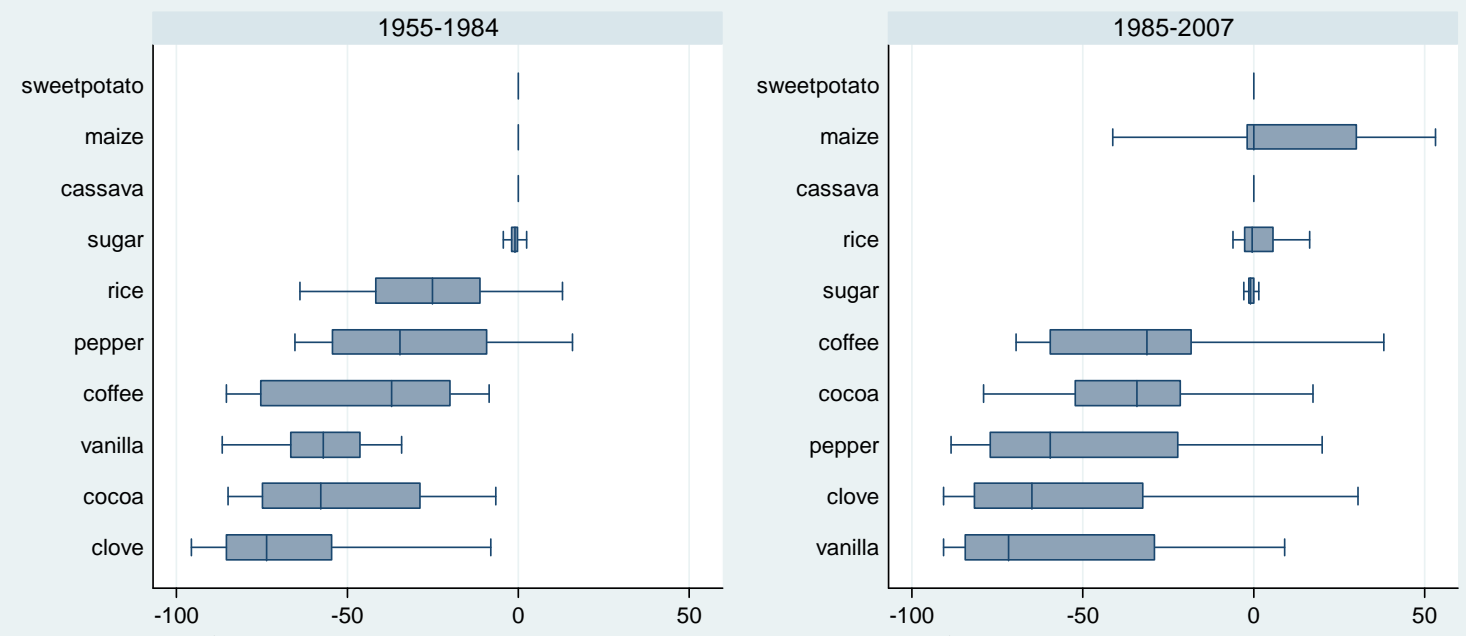

Mozambique $(\mathrm{n}=668)$
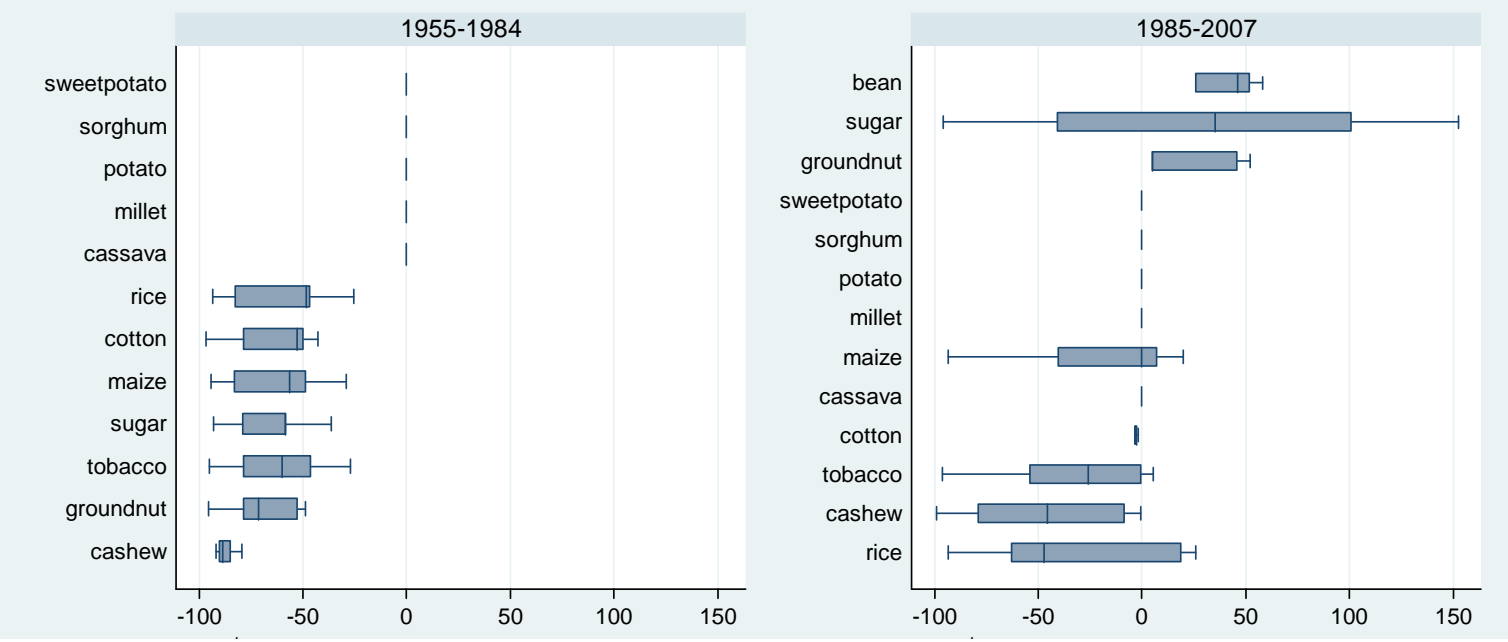
Nigeria $(n=510)$
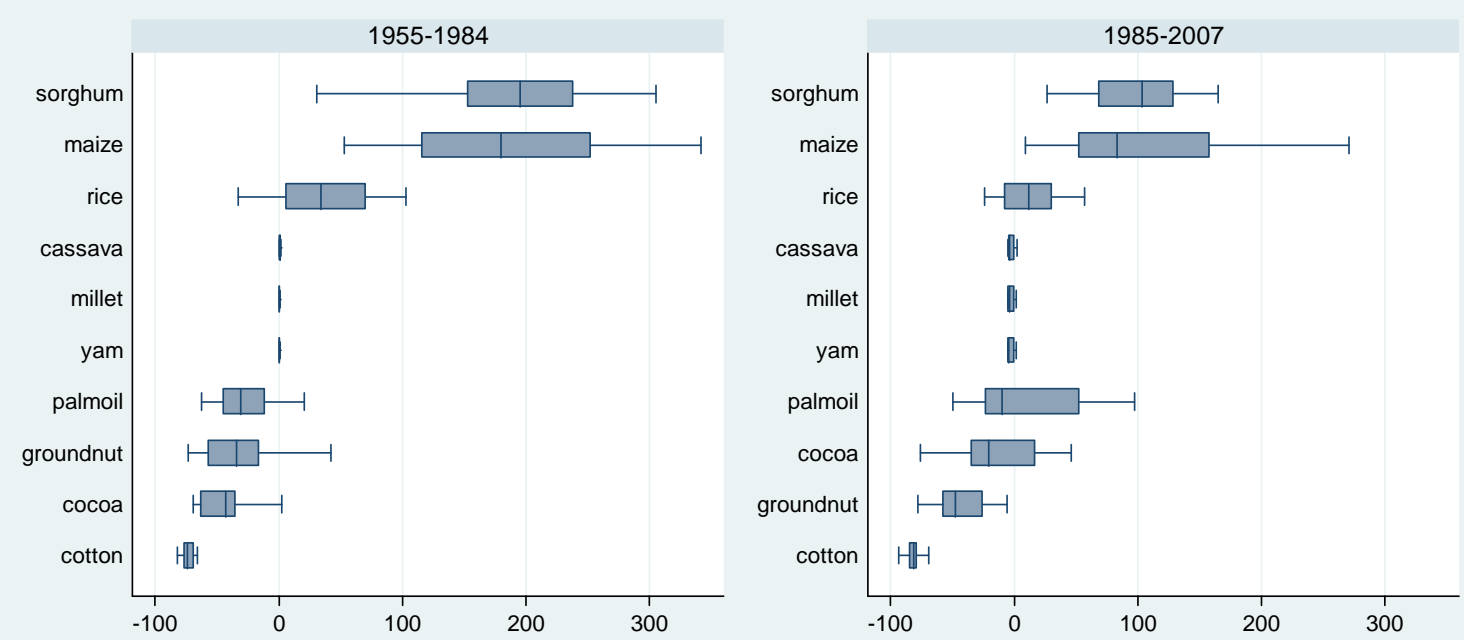

Senegal $(n=204)$
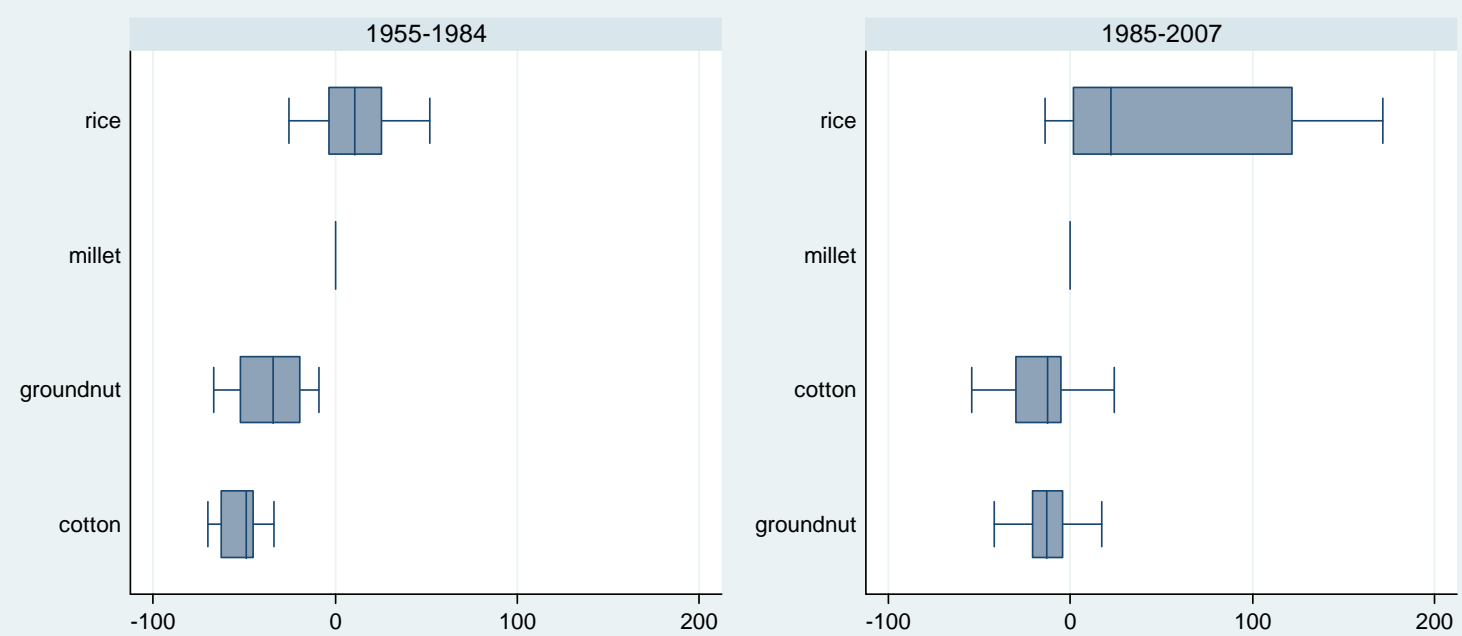

South Africa $(n=643)$
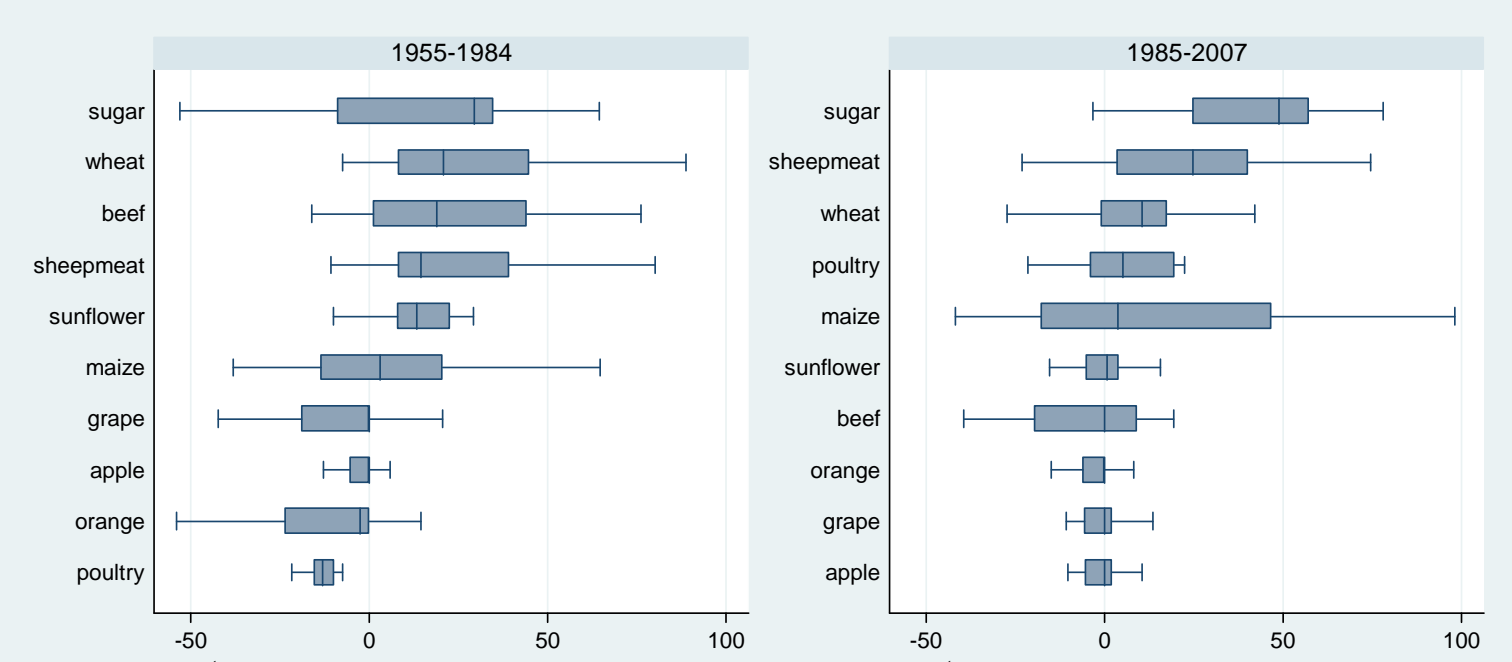
Sudan $(\mathrm{n}=611)$
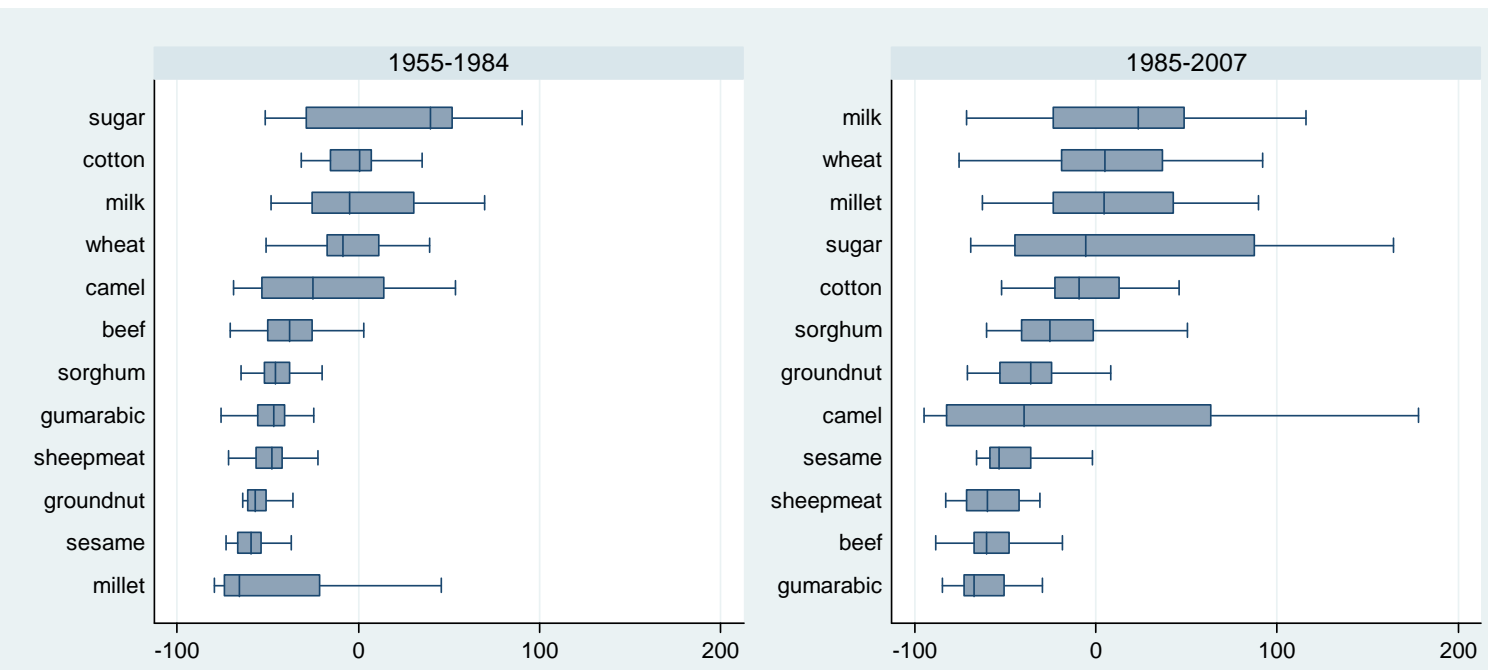

Tanzania $(\mathrm{n}=918)$
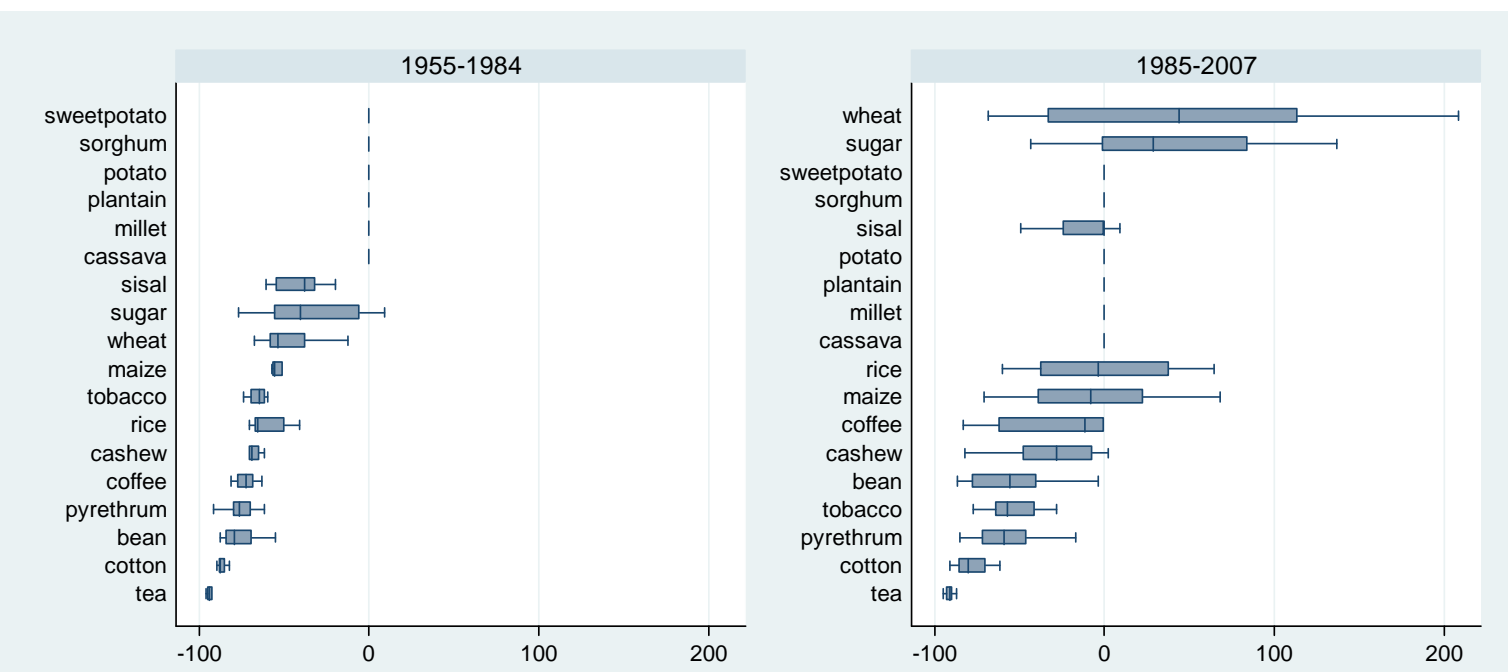

Turkey $(\mathrm{n}=609)$

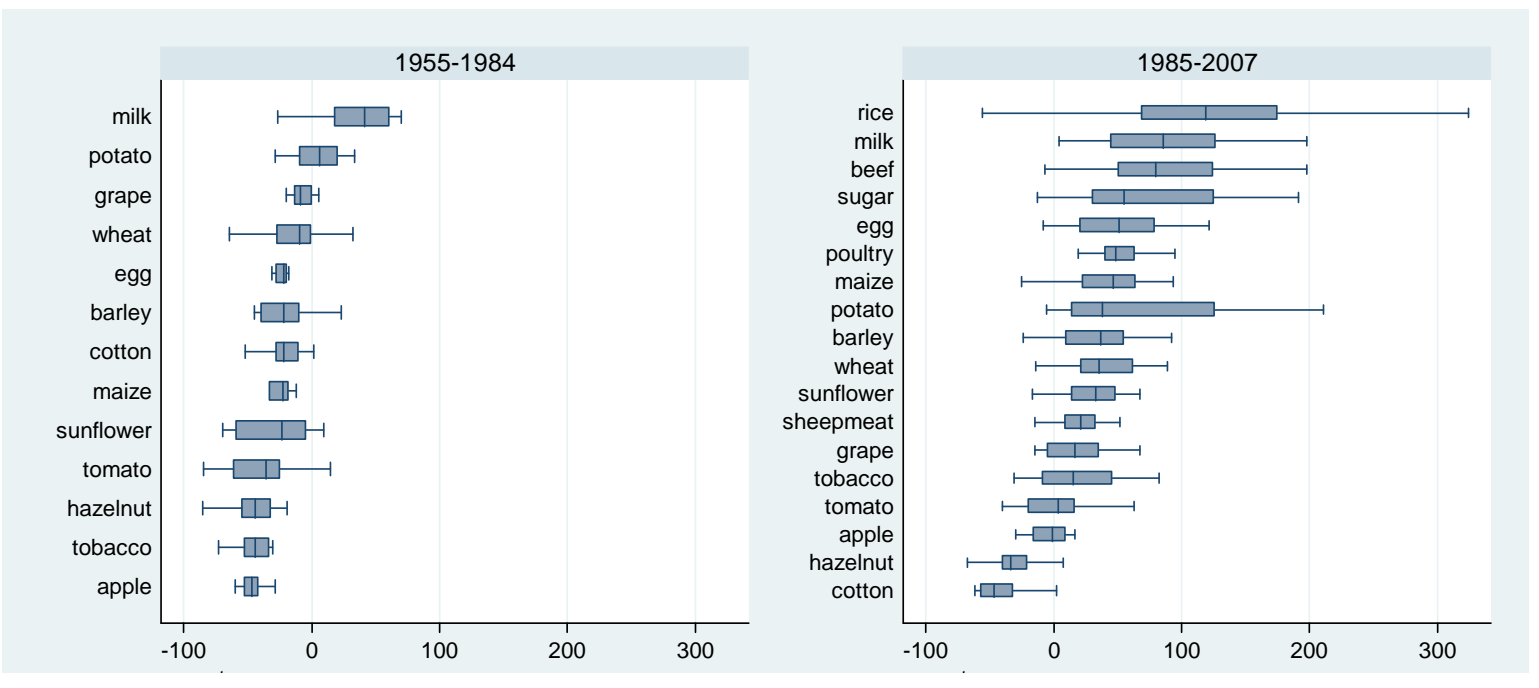


Uganda $(n=663)$
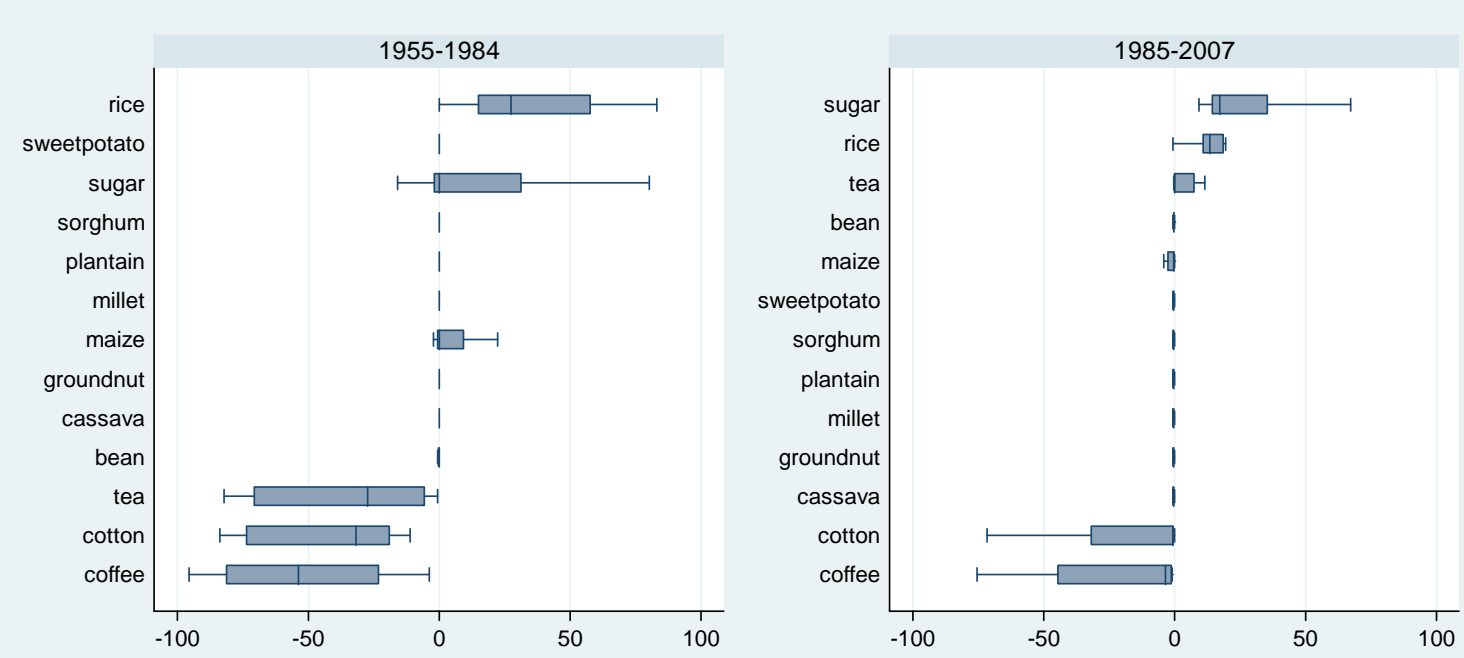

Zambia $(\mathrm{n}=510)$
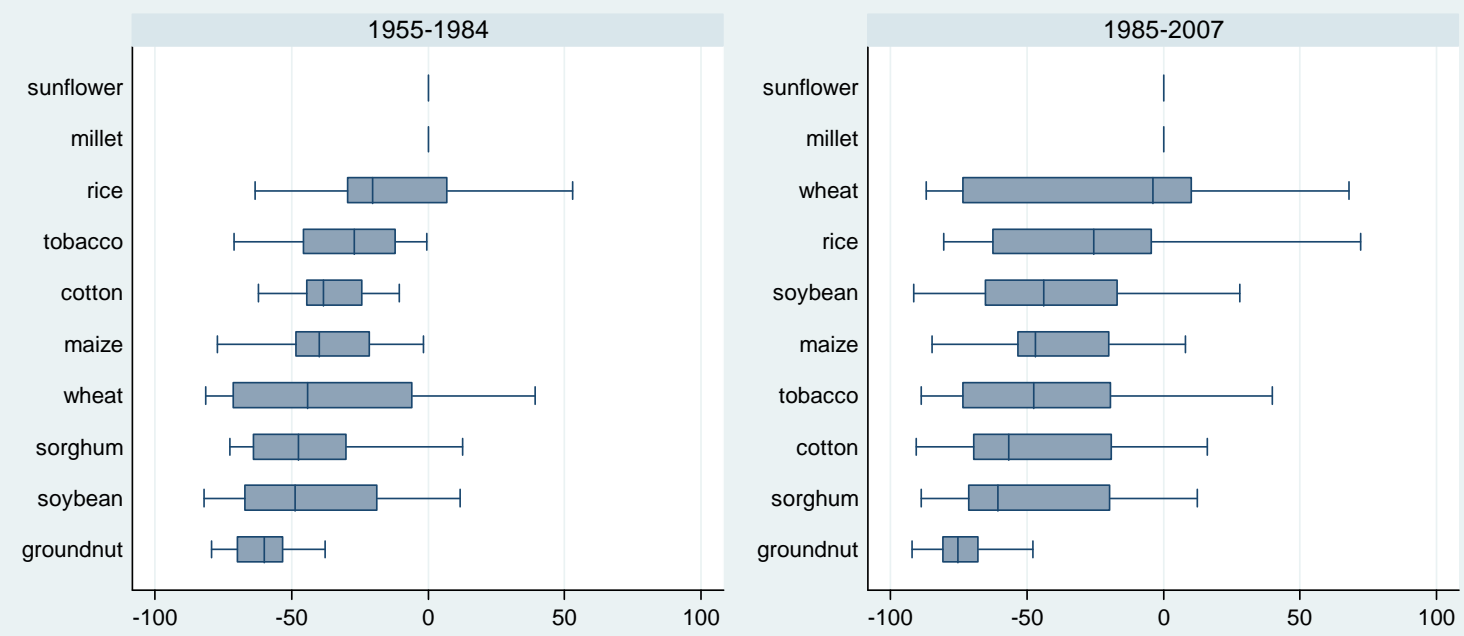

Zimbabwe ( $\mathrm{n}=408)$
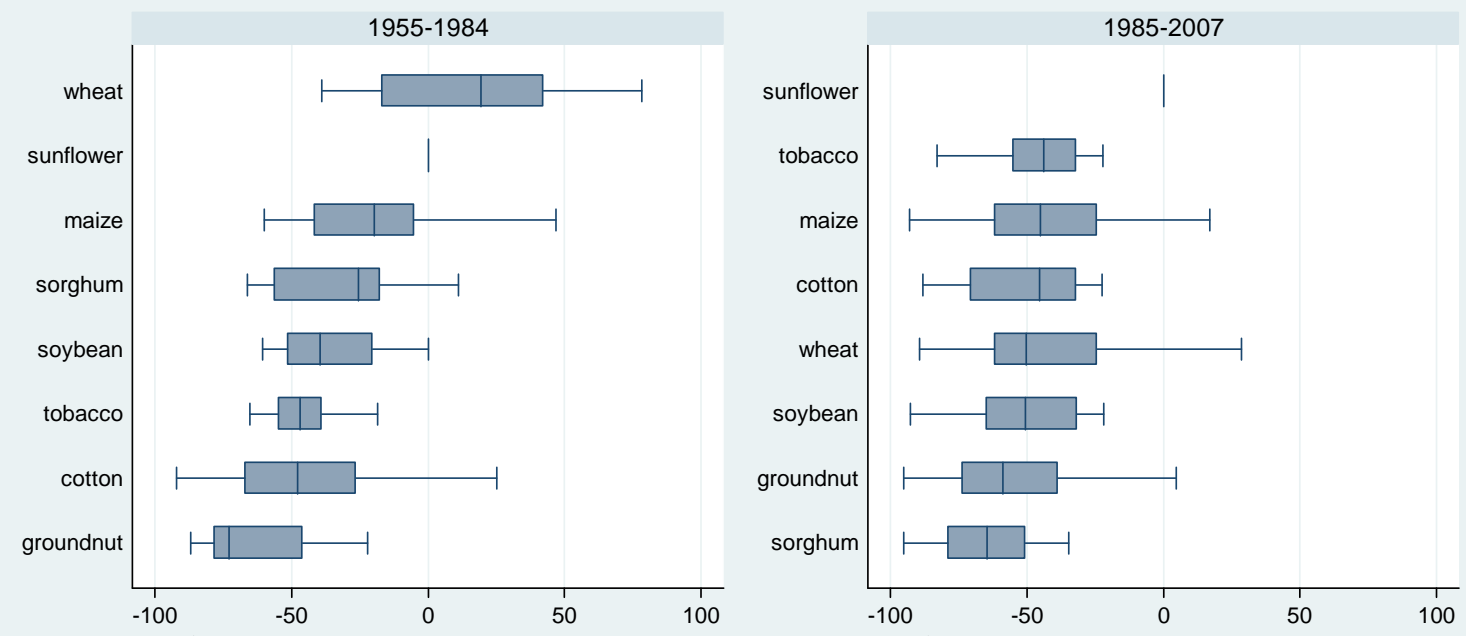
Figure A.4 (continued): Box plot distributions of NRAs of all covered agricultural products for each focus country, 1955 to 2007

(b) 12 Asian developing economies

Bangladesh $(n=306)$
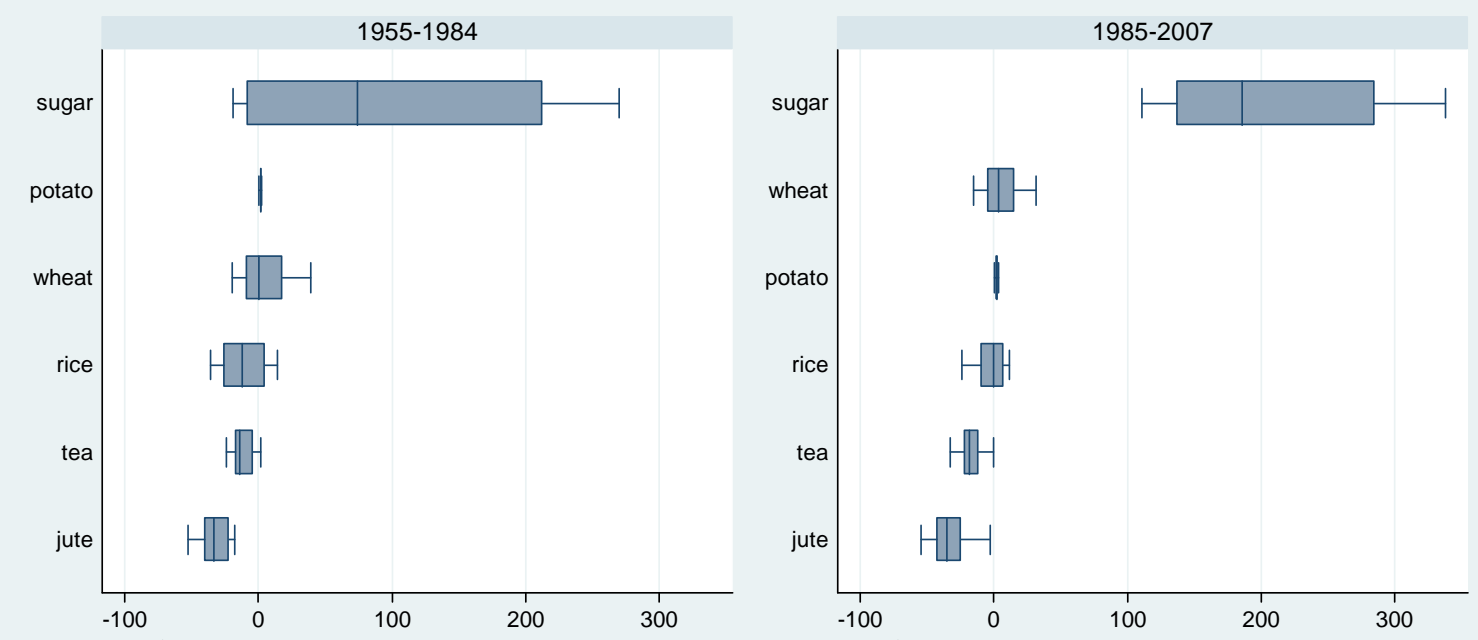

China $(\mathrm{n}=561)$
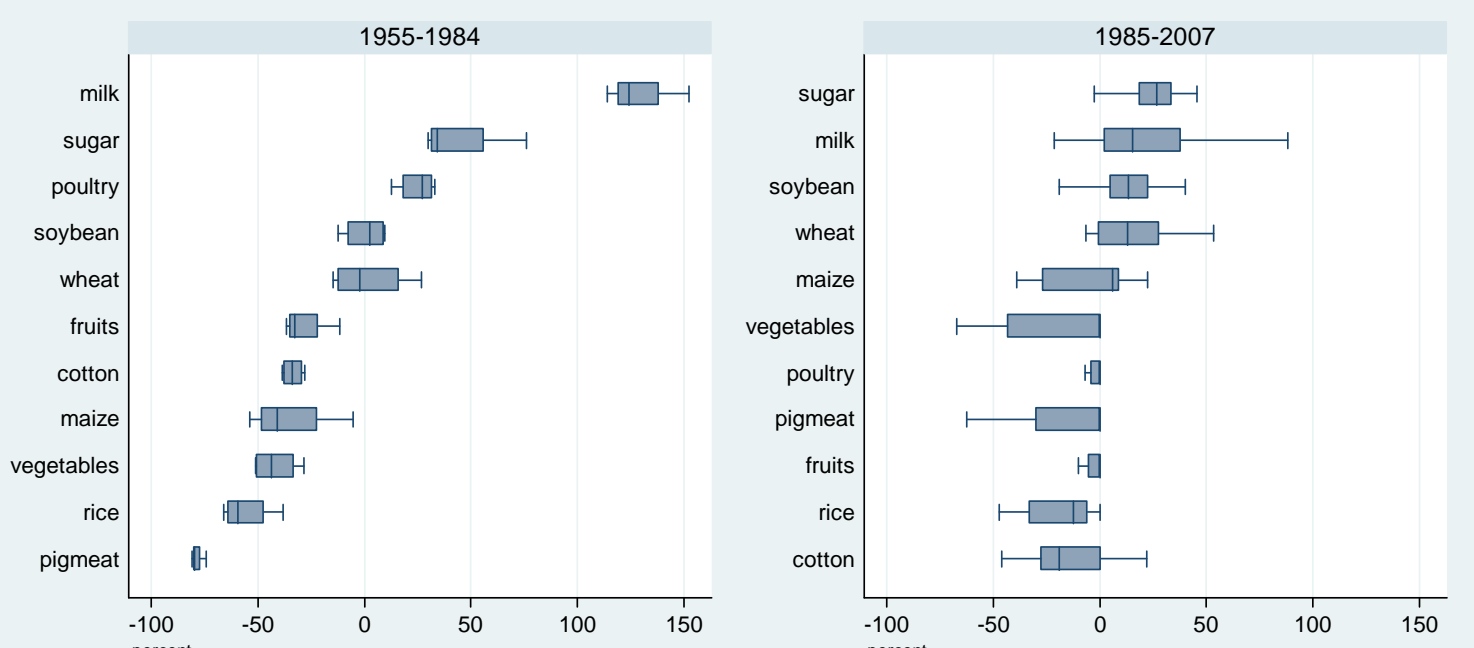

India $(n=663)$
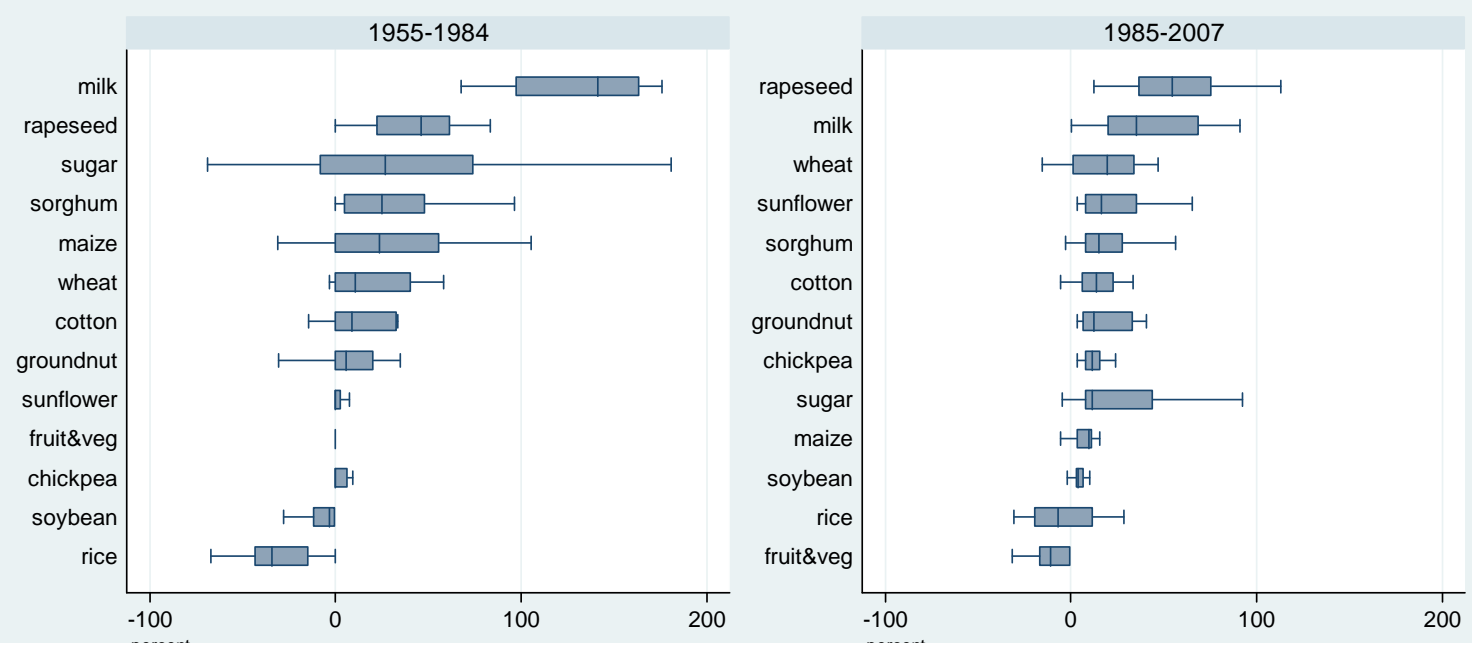
Indonesia $(\mathrm{n}=510)$
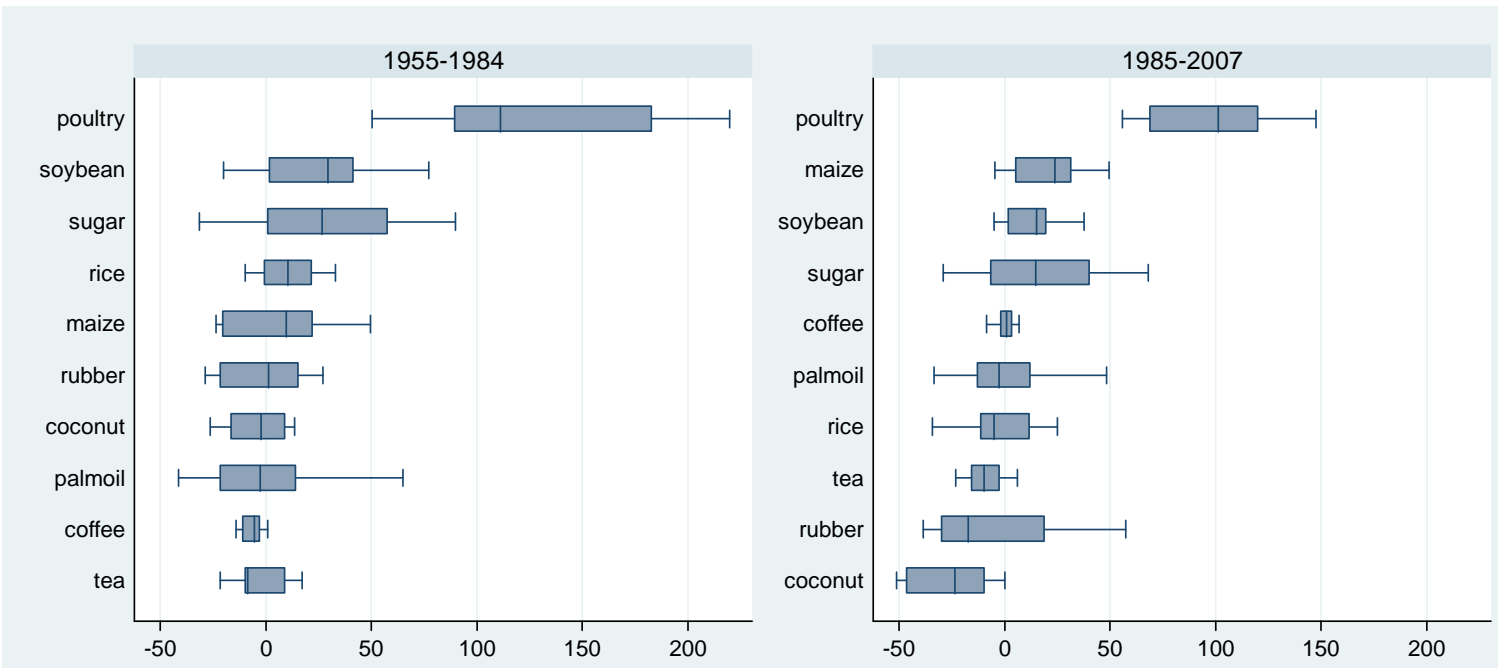

Korea $(n=634)$
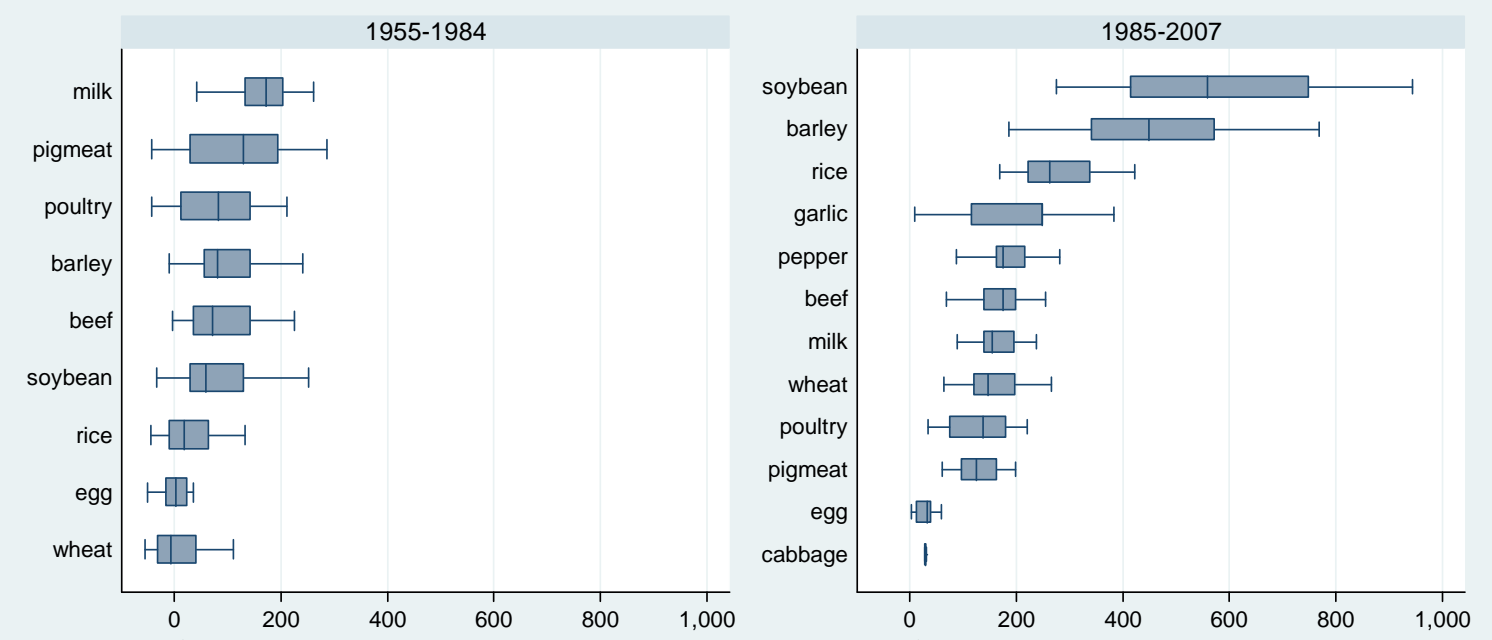

Malaysia $(n=204)$
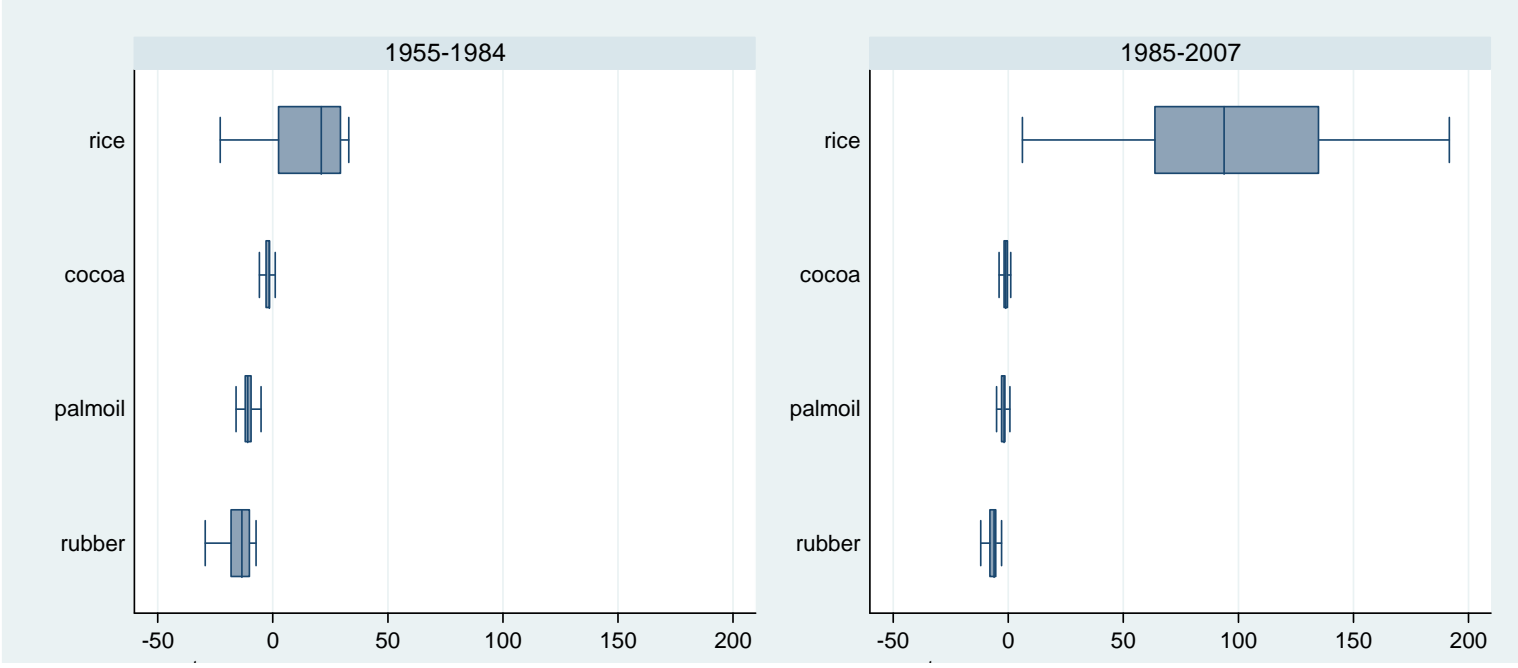
Pakistan $(\mathrm{n}=306)$
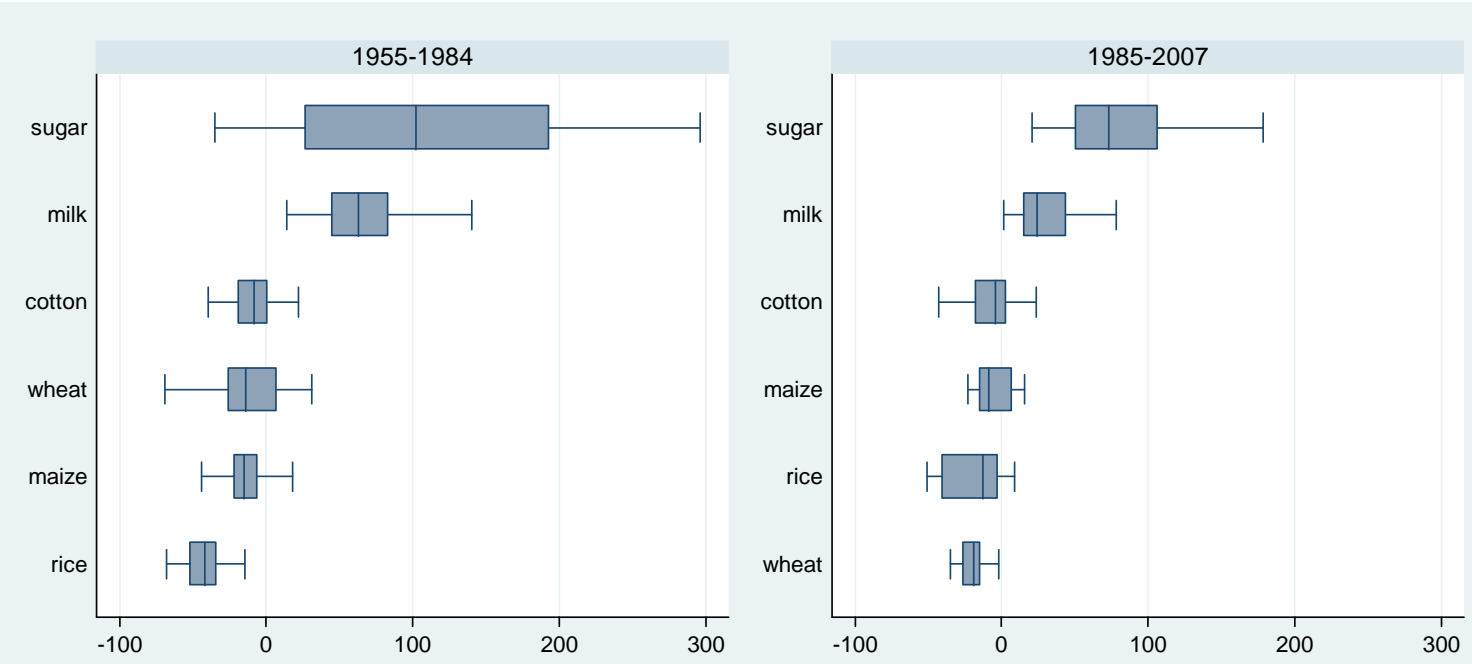

Philippines $(\mathrm{n}=$ )
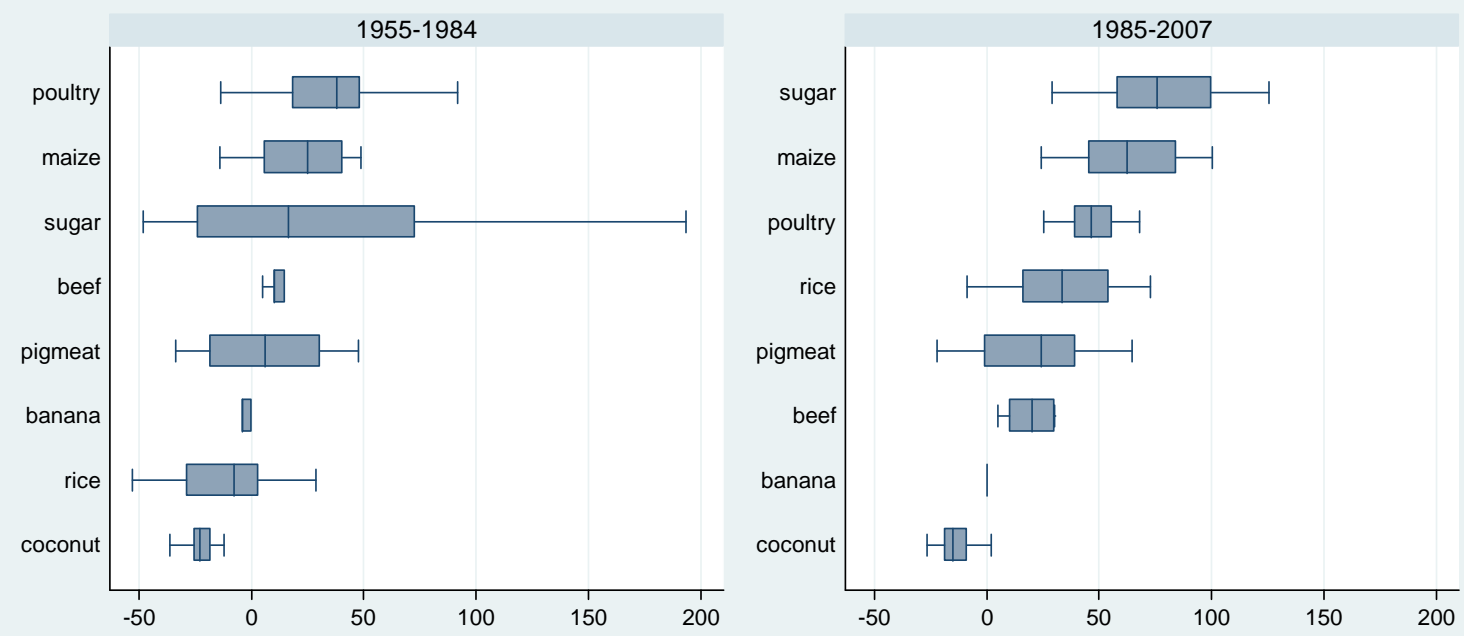

Sri Lanka $(\mathrm{n}=357)$
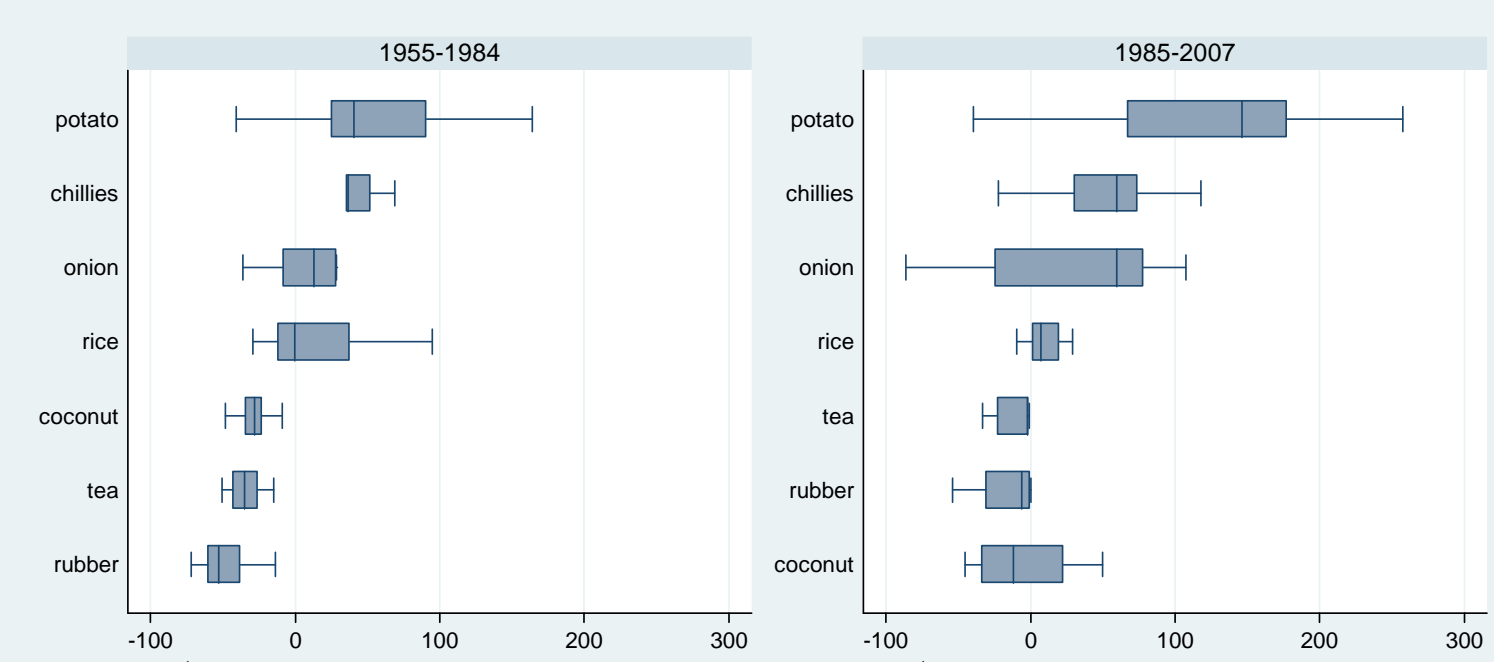
Taiwan $(\mathrm{n}=357)$
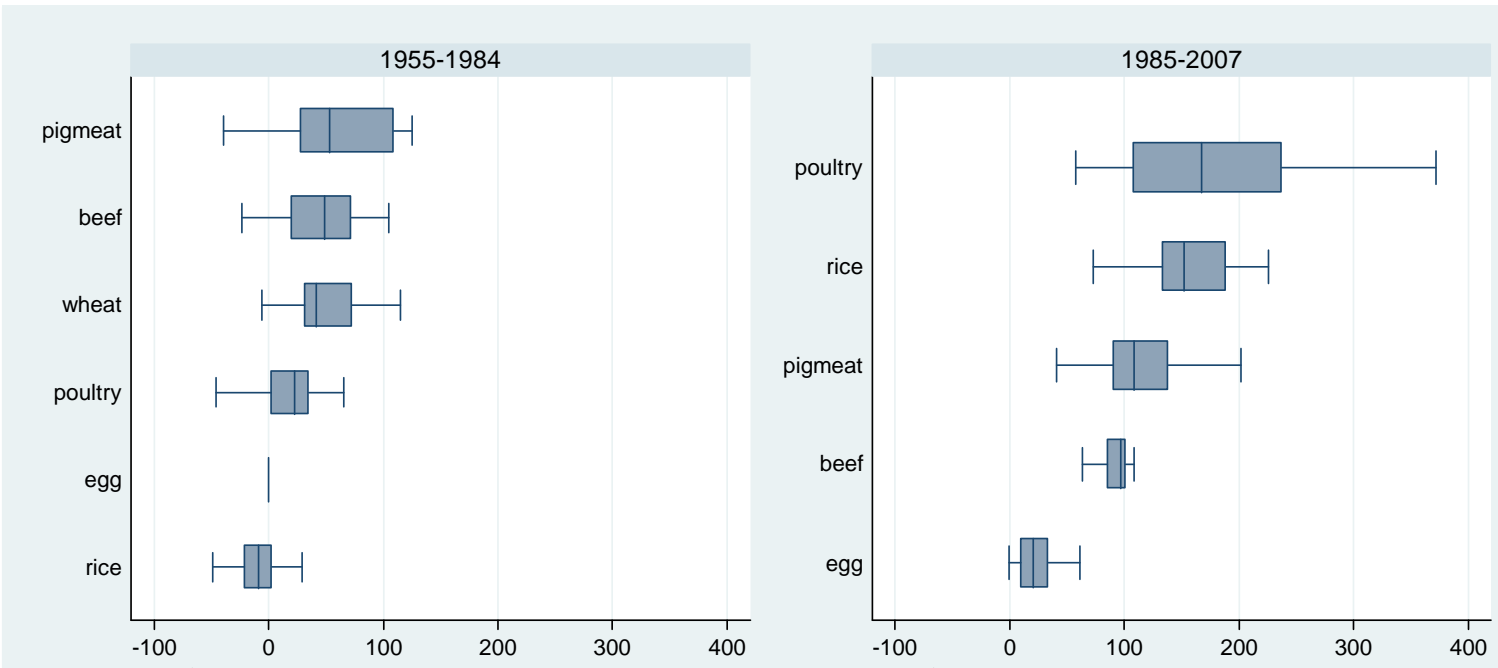

Thailand $(\mathrm{n}=459)$
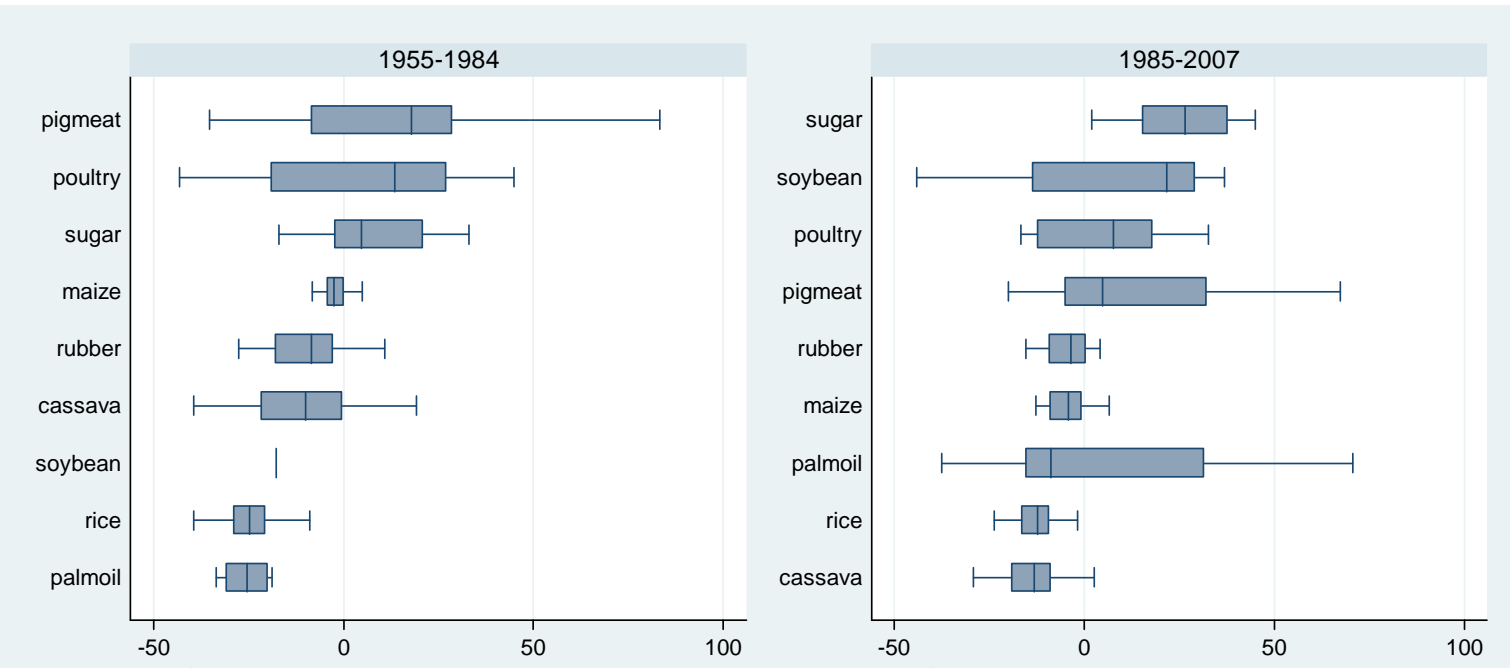

Vietnam $(n=306)$ (note: no data in 1955-85)

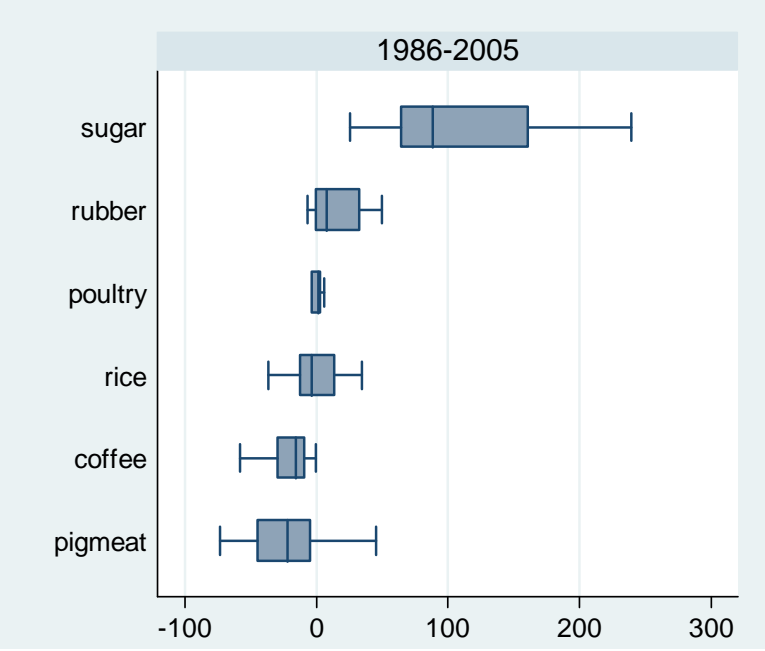


Figure A.4 (continued): Box plot distributions of NRAs of all covered agricultural products for each focus country, 1955 to 2007

(c) 8 Latin American countries

Argentina $(\mathrm{n}=306)$
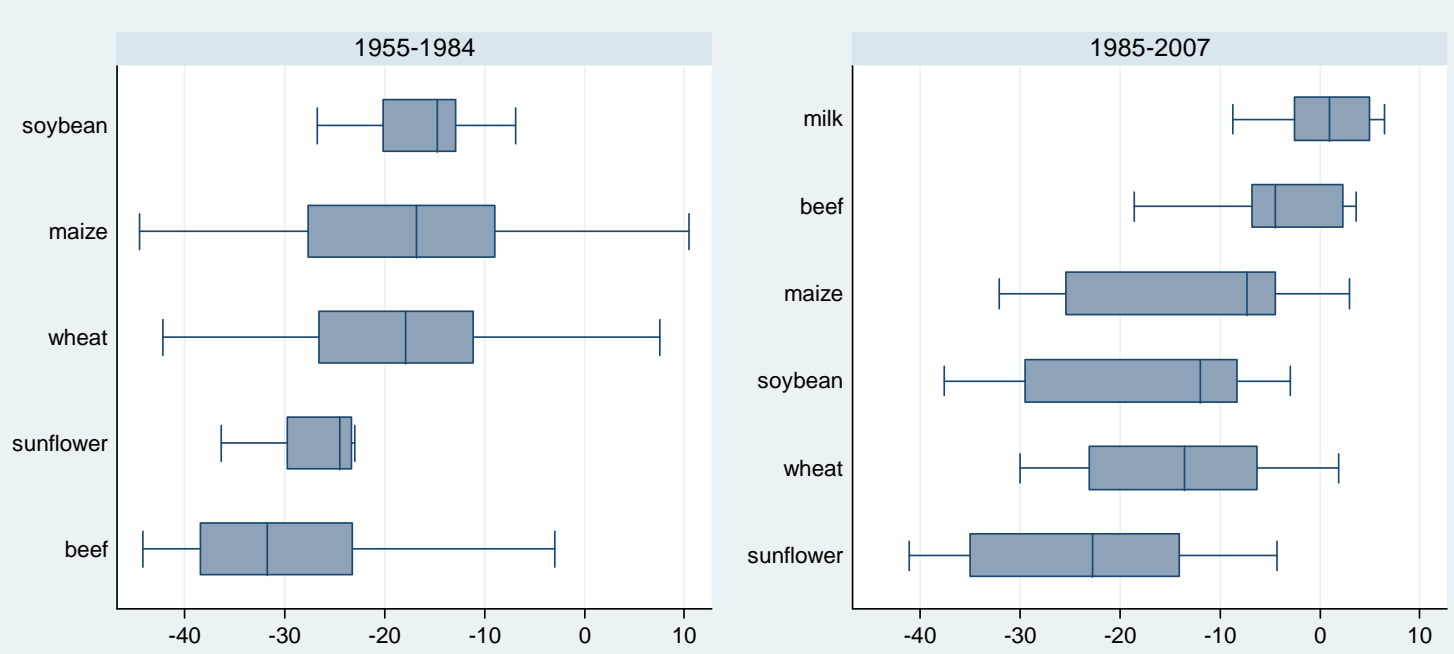

Brazil $(\mathrm{n}=510)$
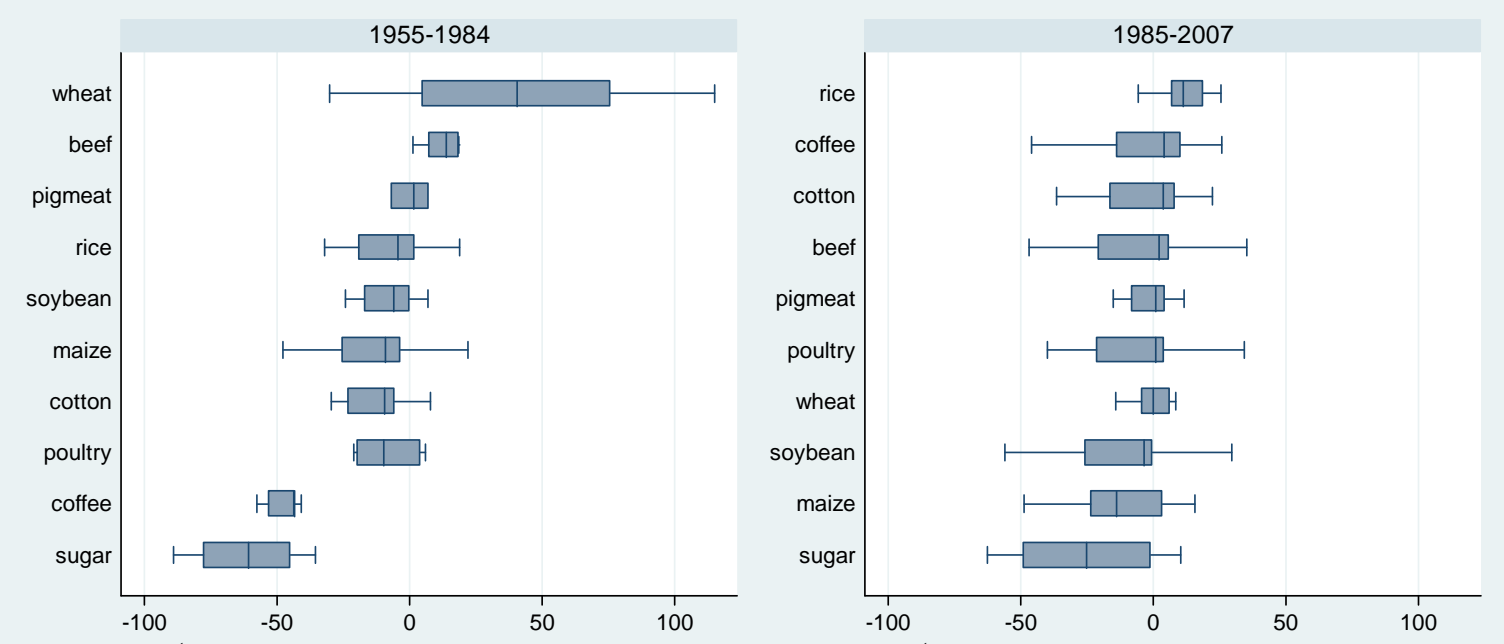

Chile $(\mathrm{n}=357)$
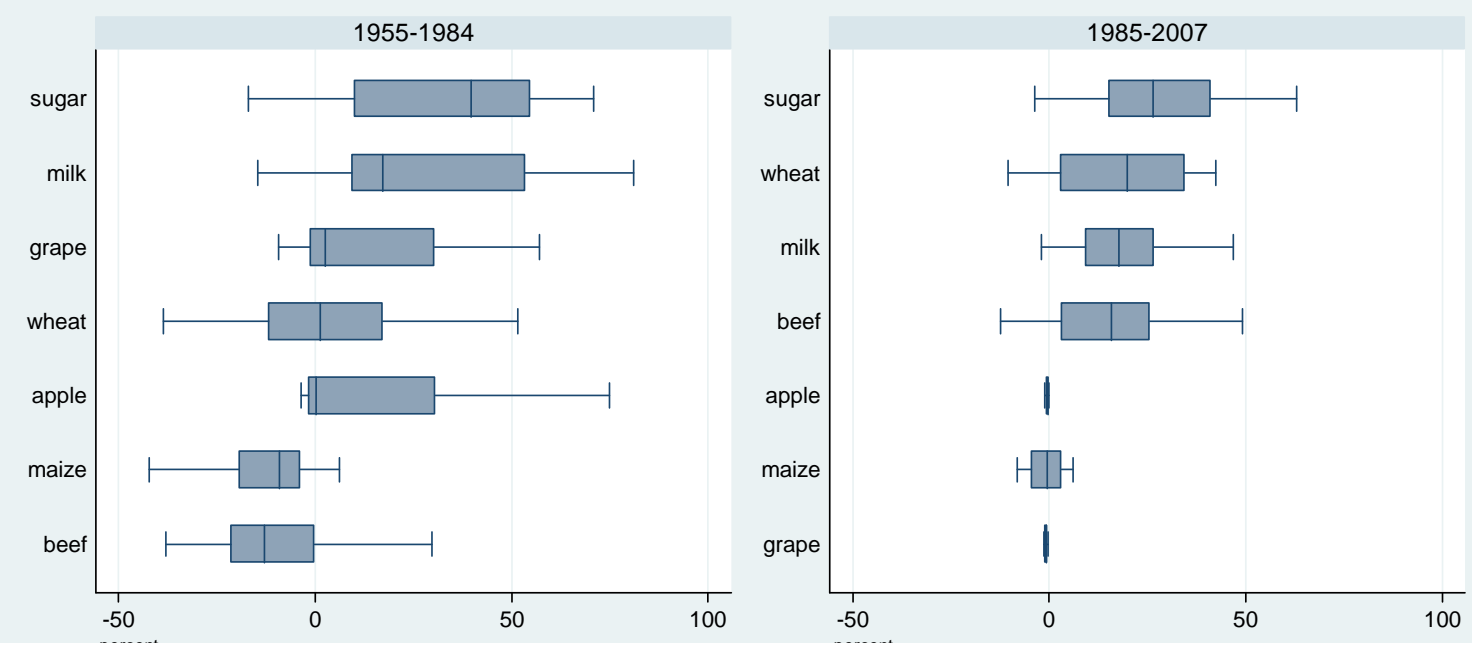
Colombia $(\mathrm{n}=561)$
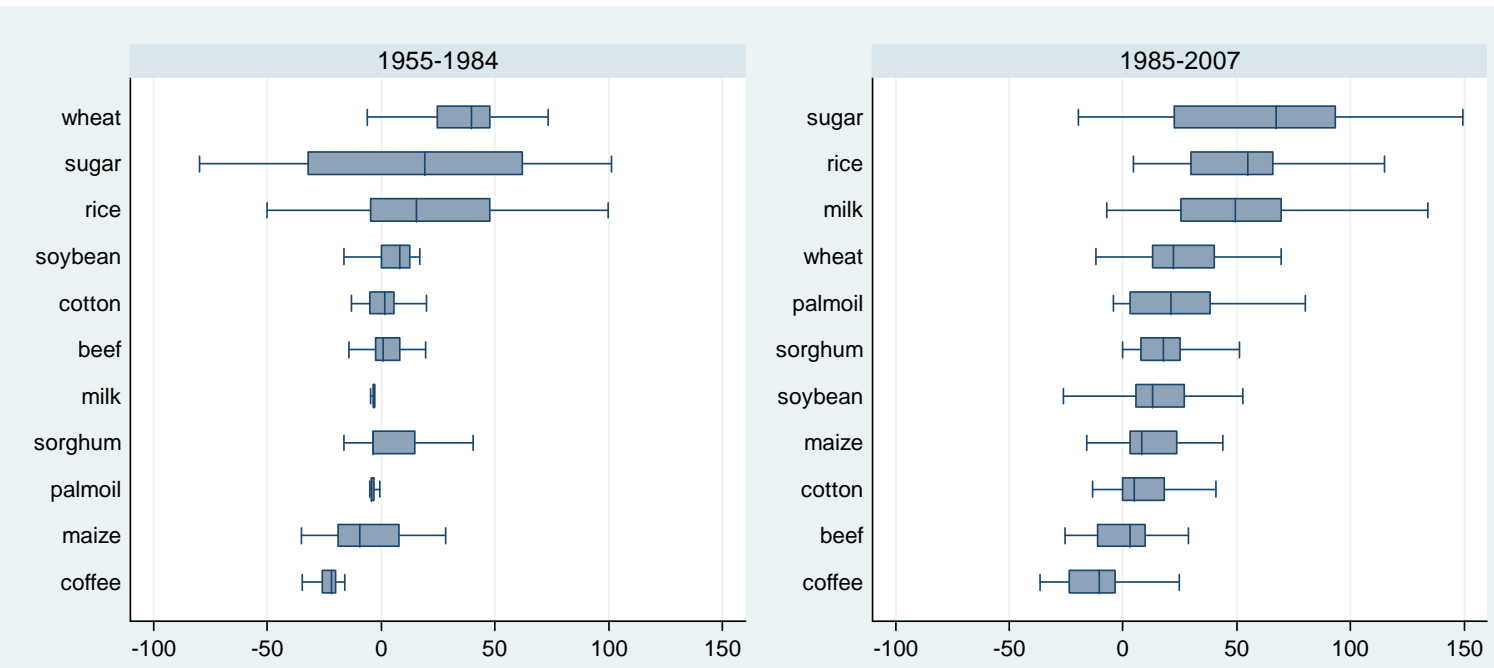

Dominican Republic $(\mathrm{n}=510)$
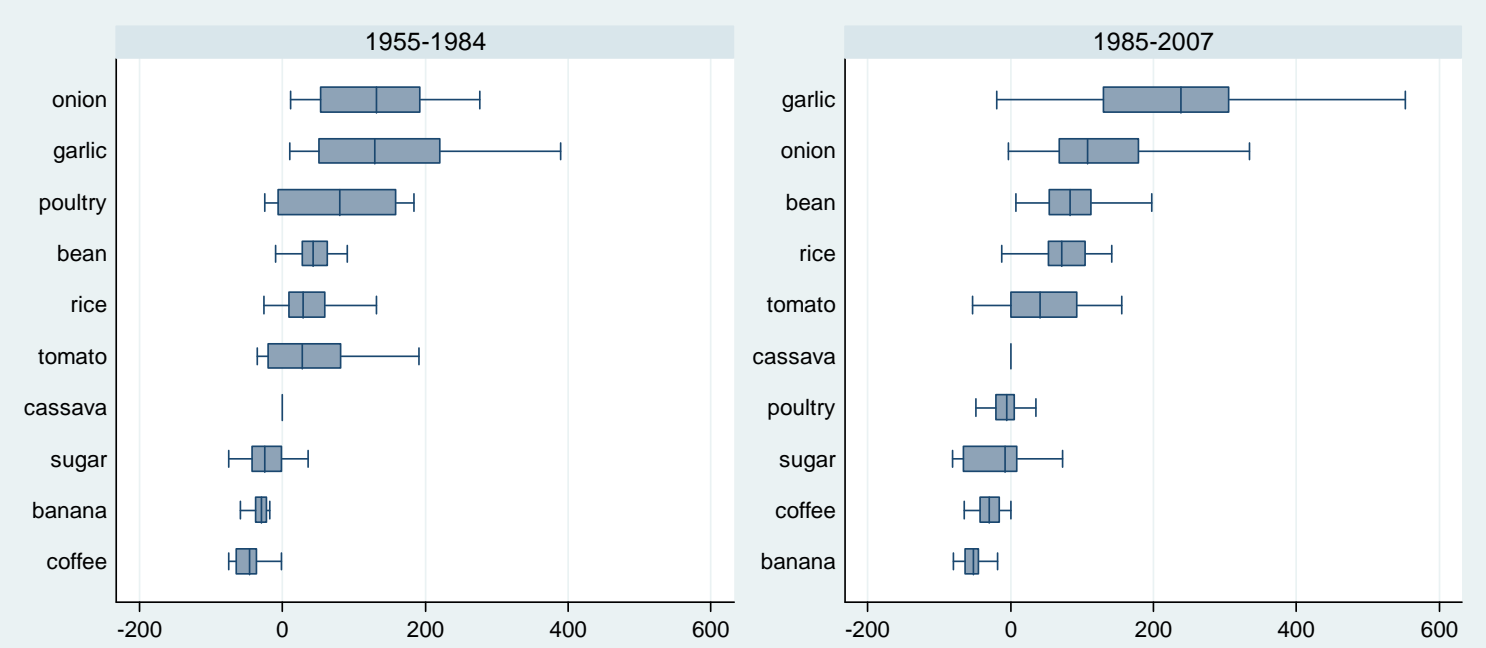

Ecuador $(\mathrm{n}=561)$
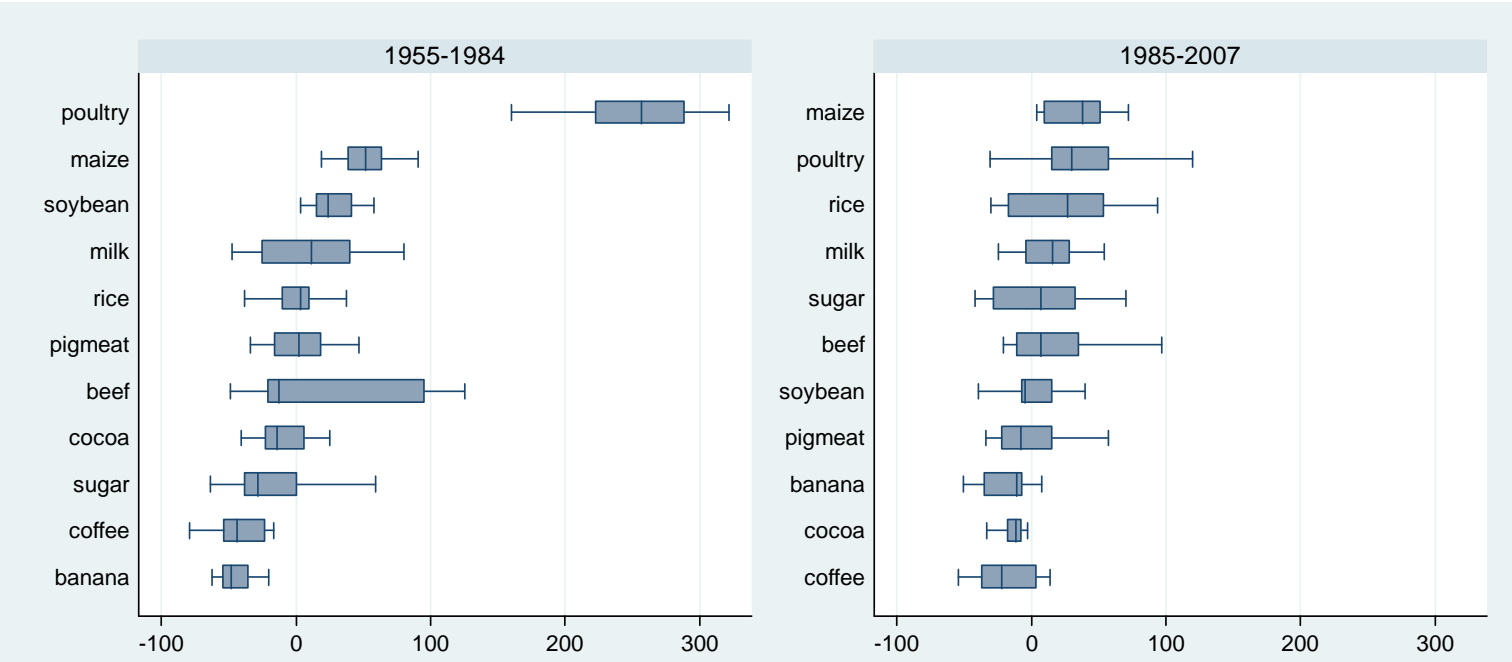
$\operatorname{Mexico~}(\mathrm{n}=793)$
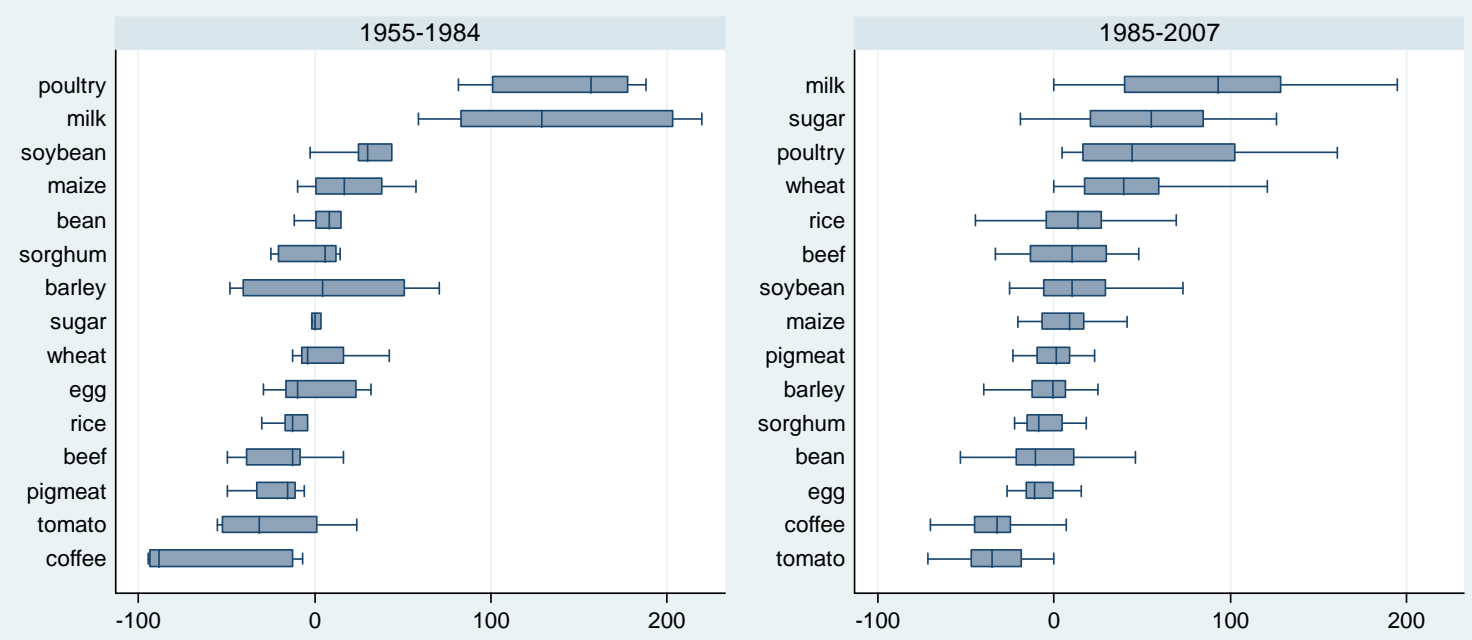

Nicaragua $(n=612)$ (note: Nicaragua only has data for 1993 to 2004)

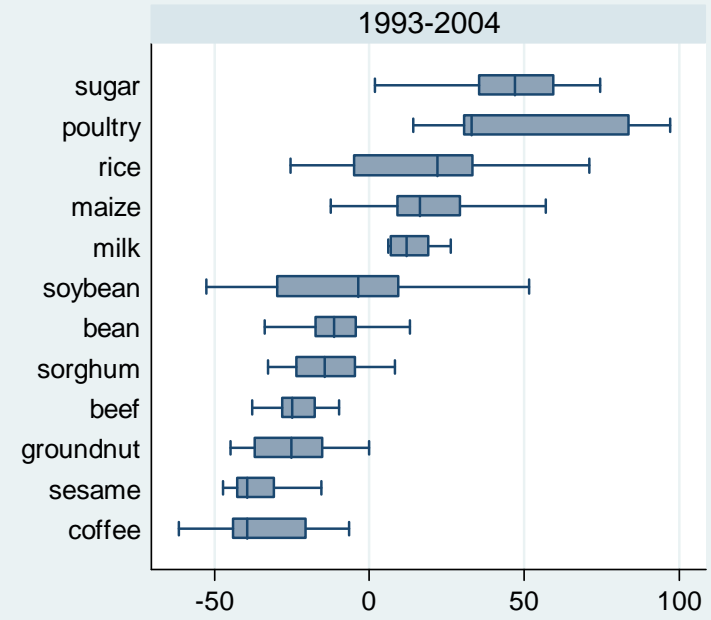


Figure A.4 (continued): Box plot distributions of NRAs of covered agricultural products for each focus country, 1955 to 2007

(d) 12 European transition economies

Bulgaria $(n=183)$
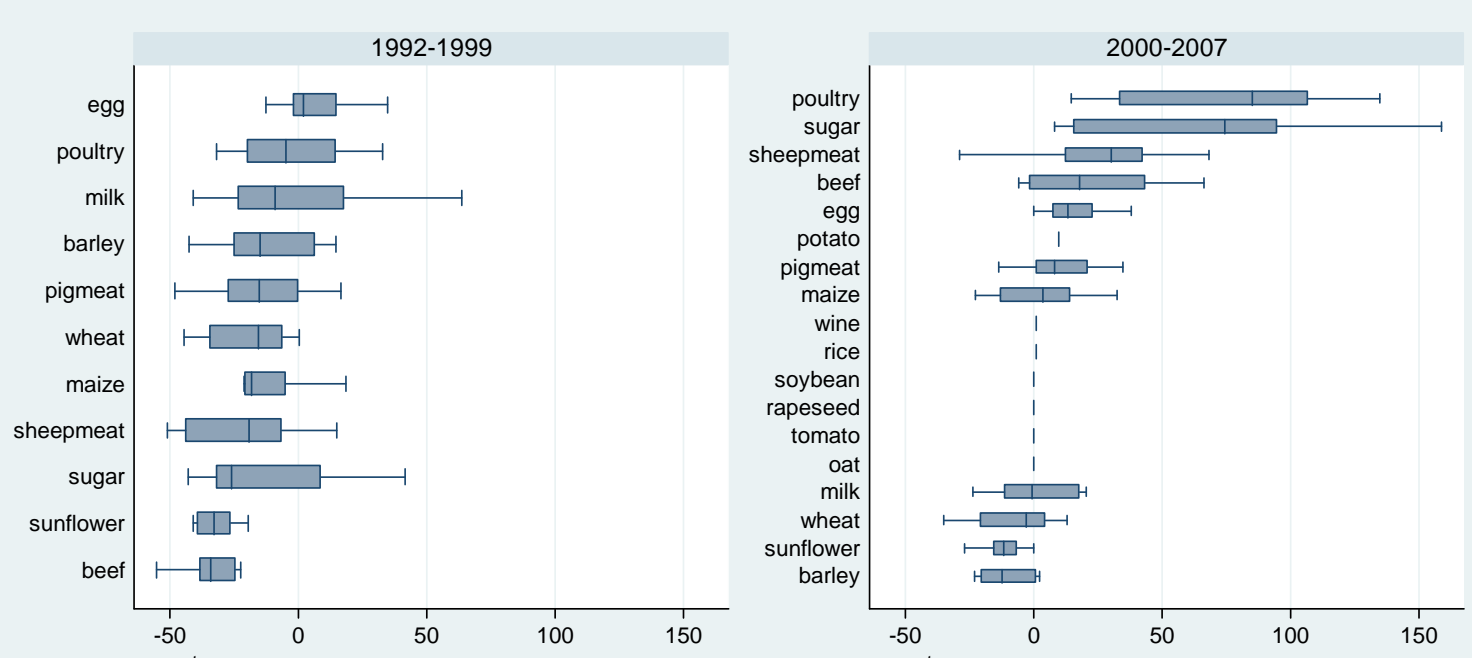

Czech Rep. $(\mathrm{n}=165)$
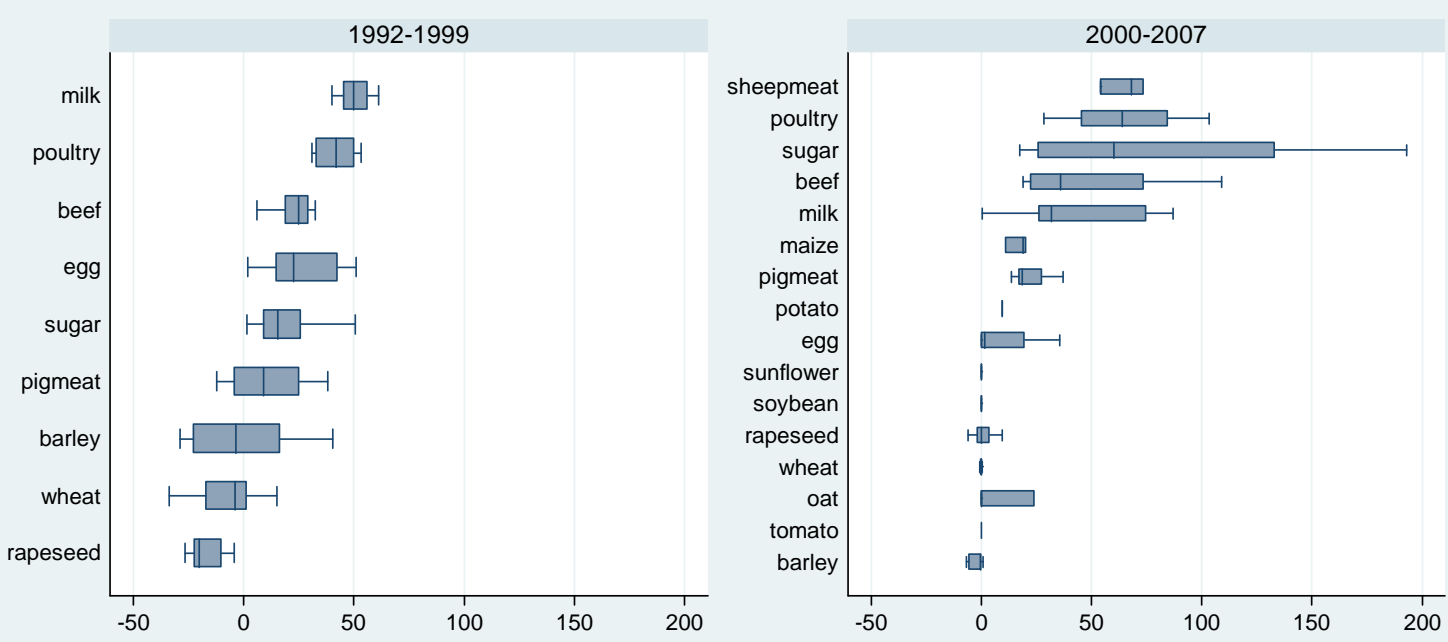

Estonia $(\mathrm{n}=167)$
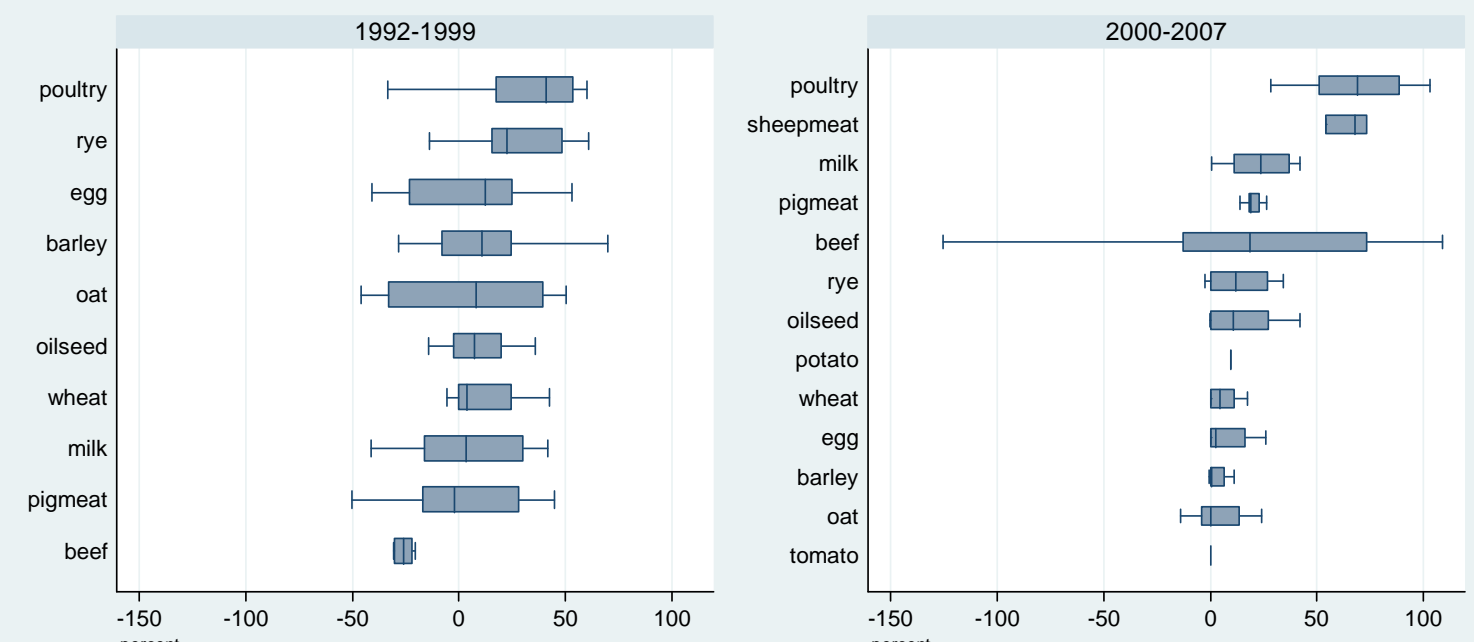
Hungary $(n=210)$
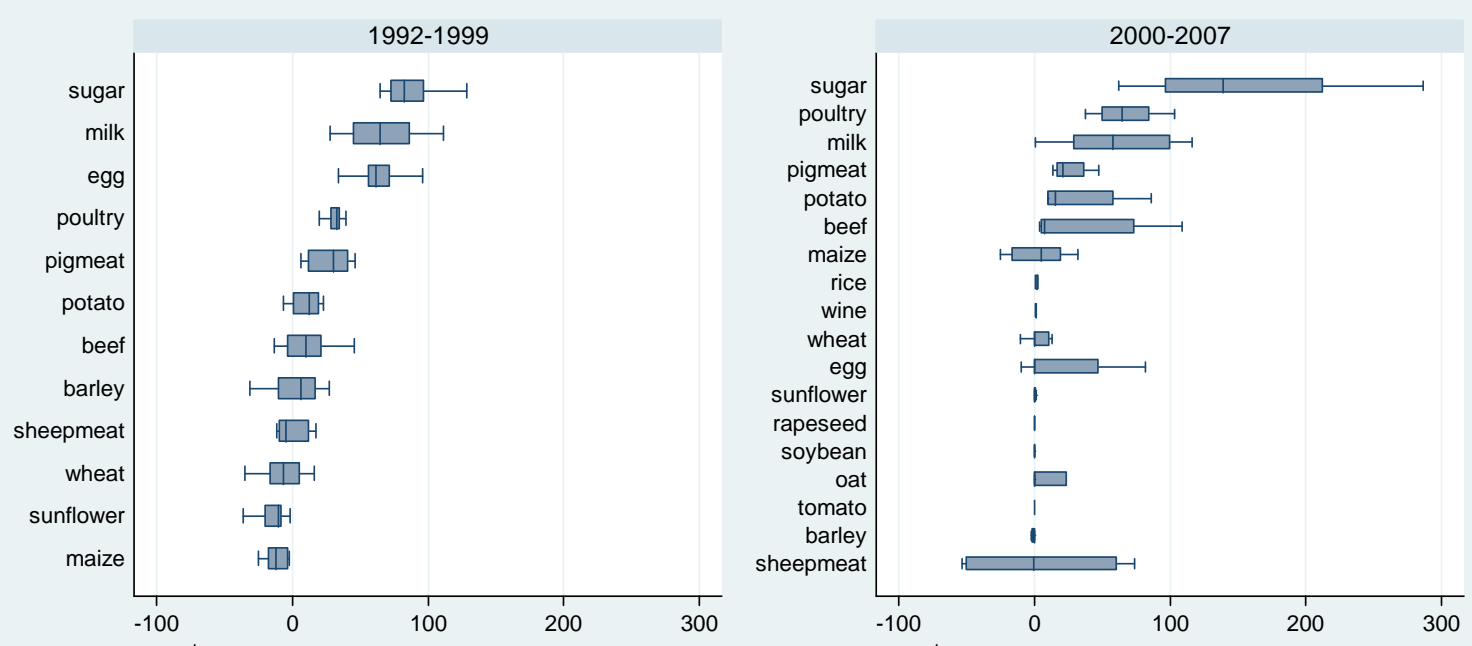

Kazakhstan $(\mathrm{n}=30)$

Latvia $(\mathrm{n}=181)$
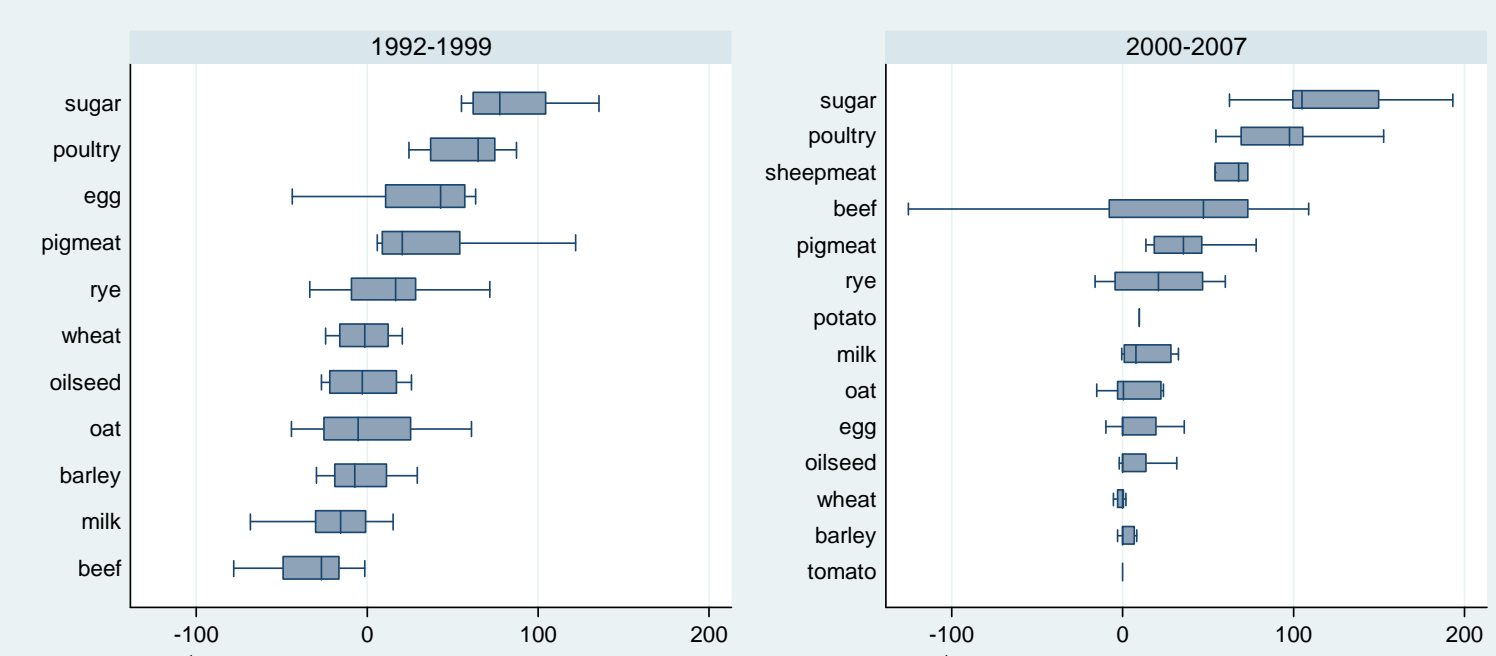
Lithuania (n = 184)
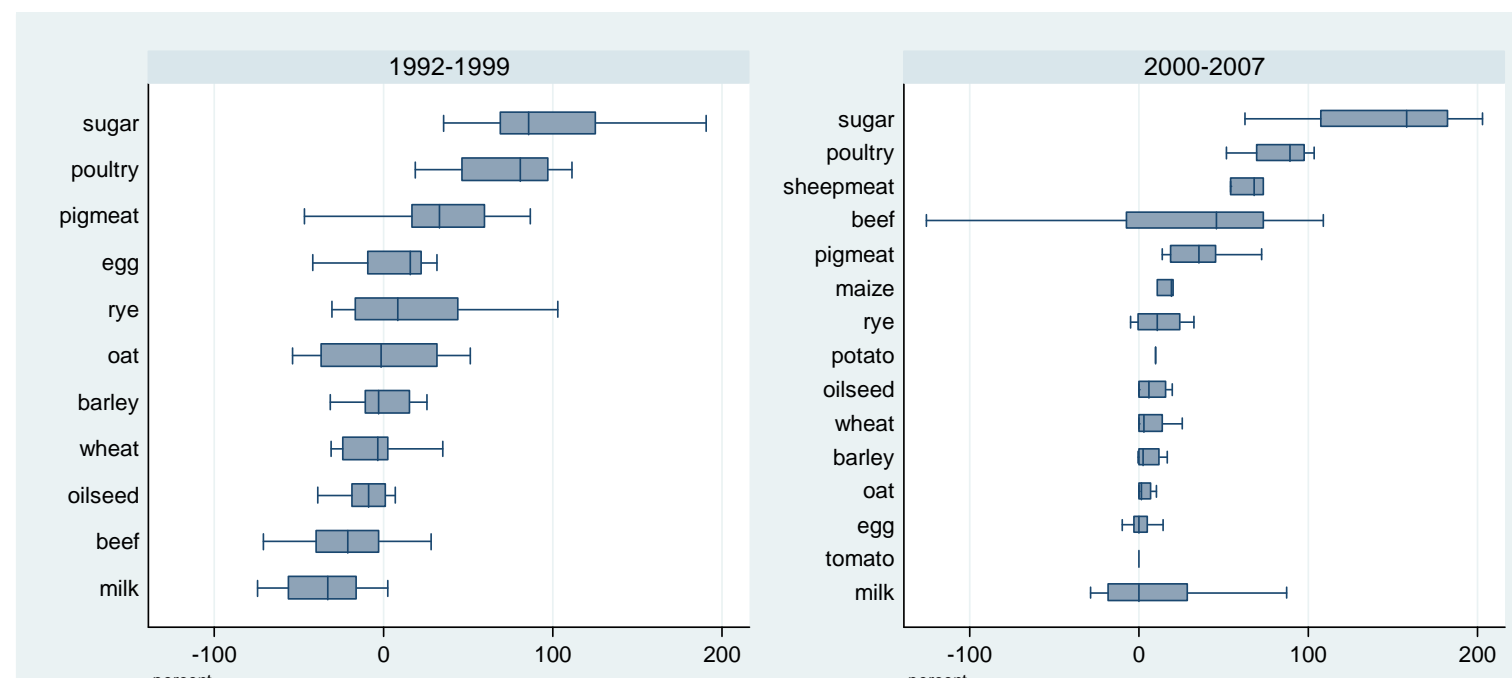

Poland $(\mathrm{n}=186)$
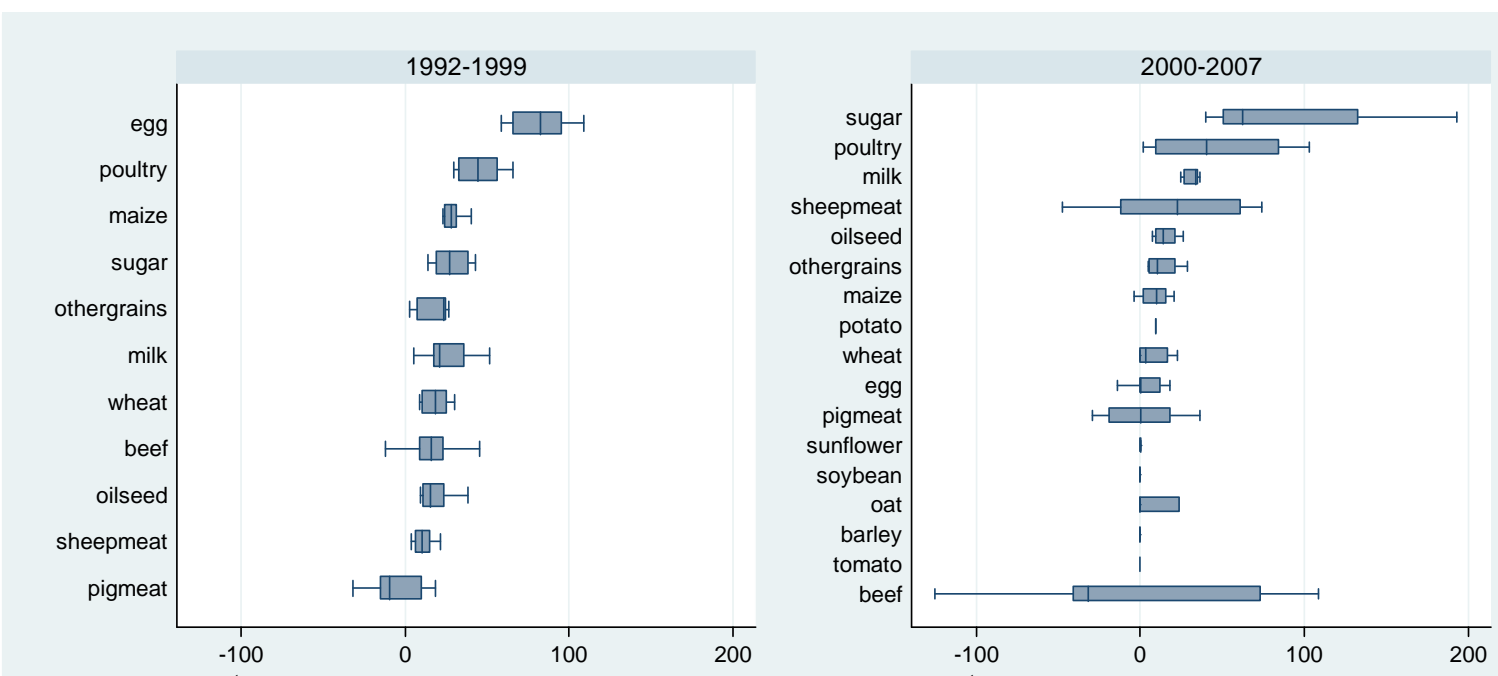

Romania $(\mathrm{n}=228)$

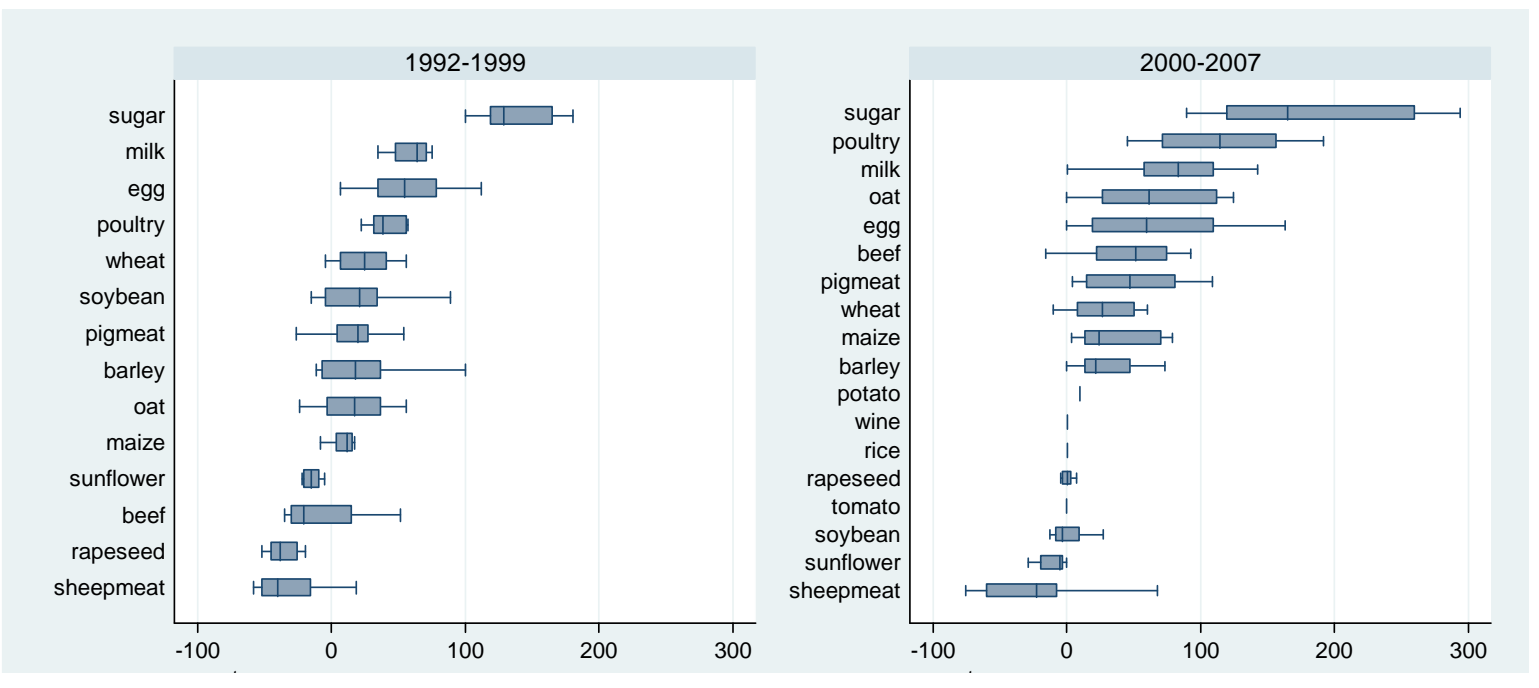


Russia (n = 168)
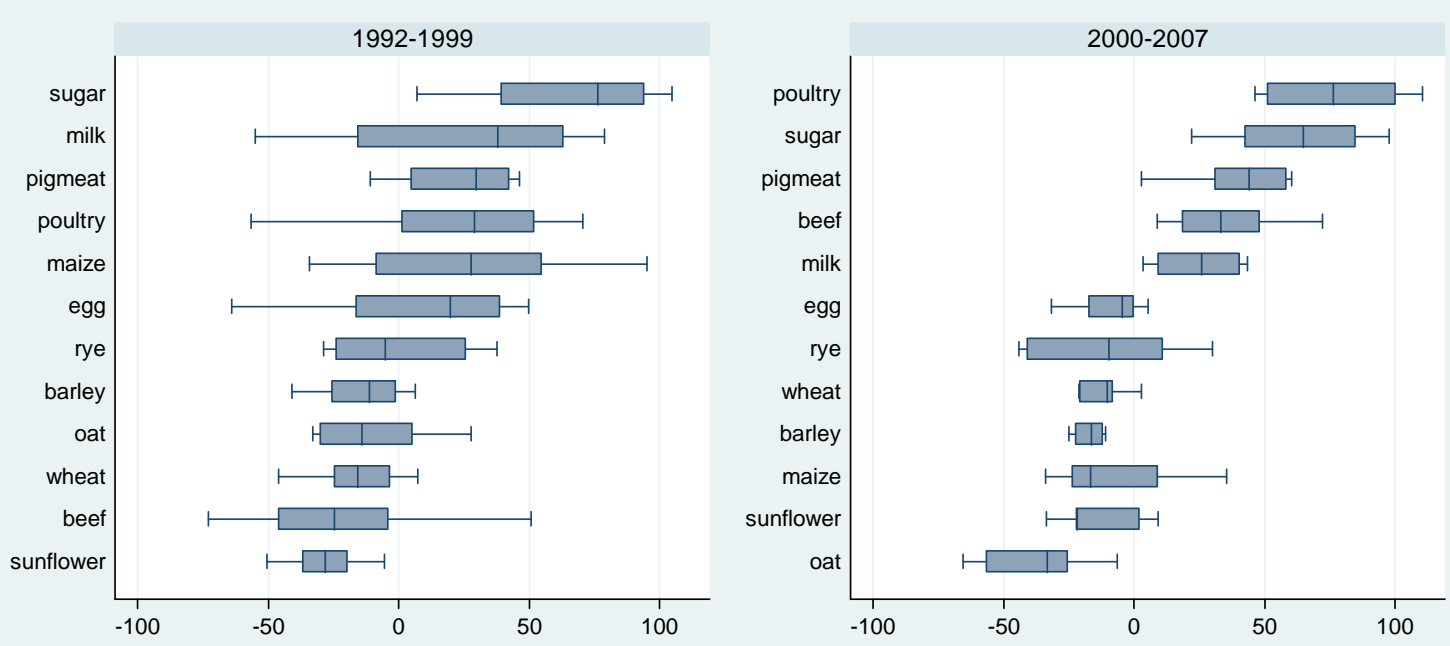

Slovakia $(\mathrm{n}=219)$
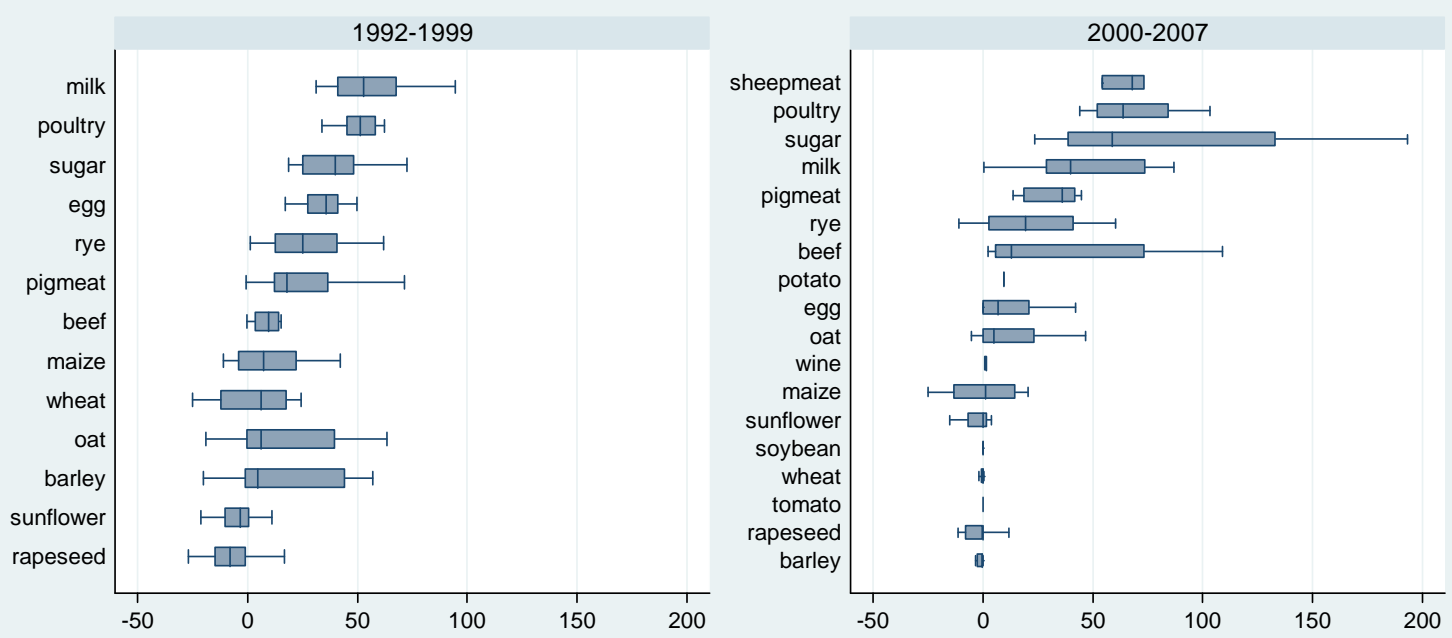

Slovenia $(\mathrm{n}=159)$
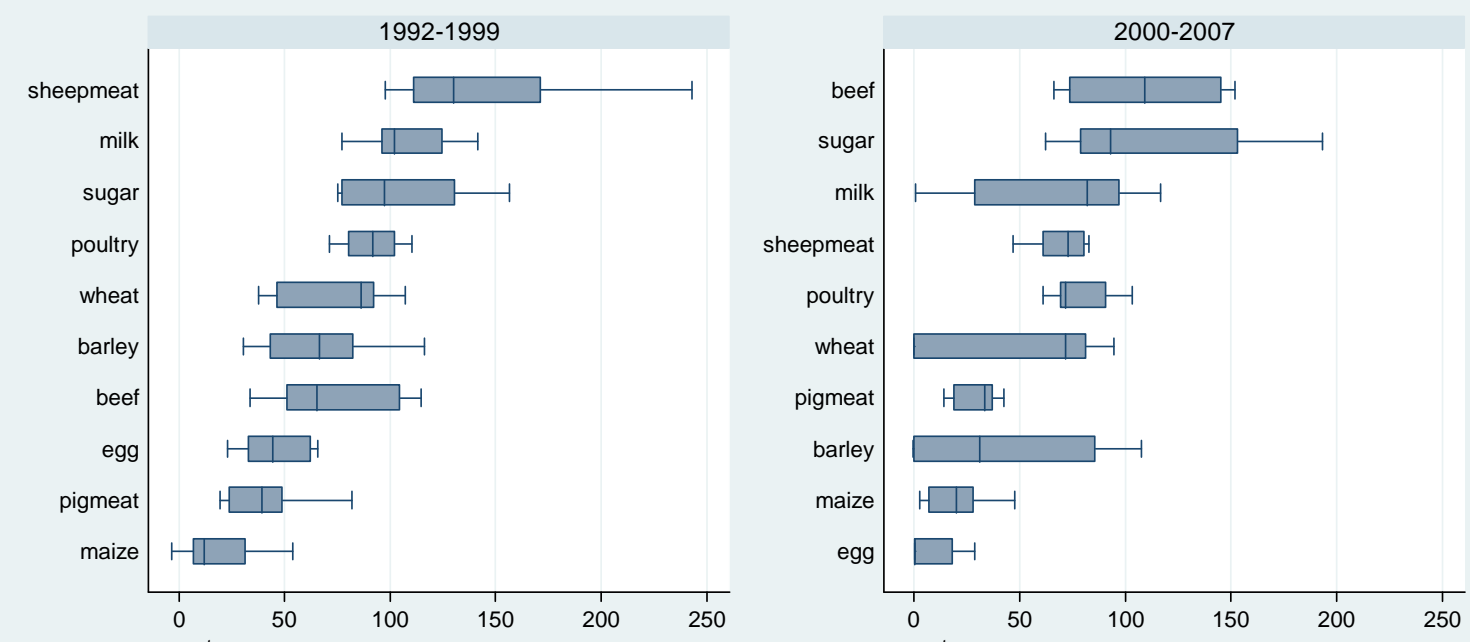
Ukraine $(\mathrm{n}=178)$
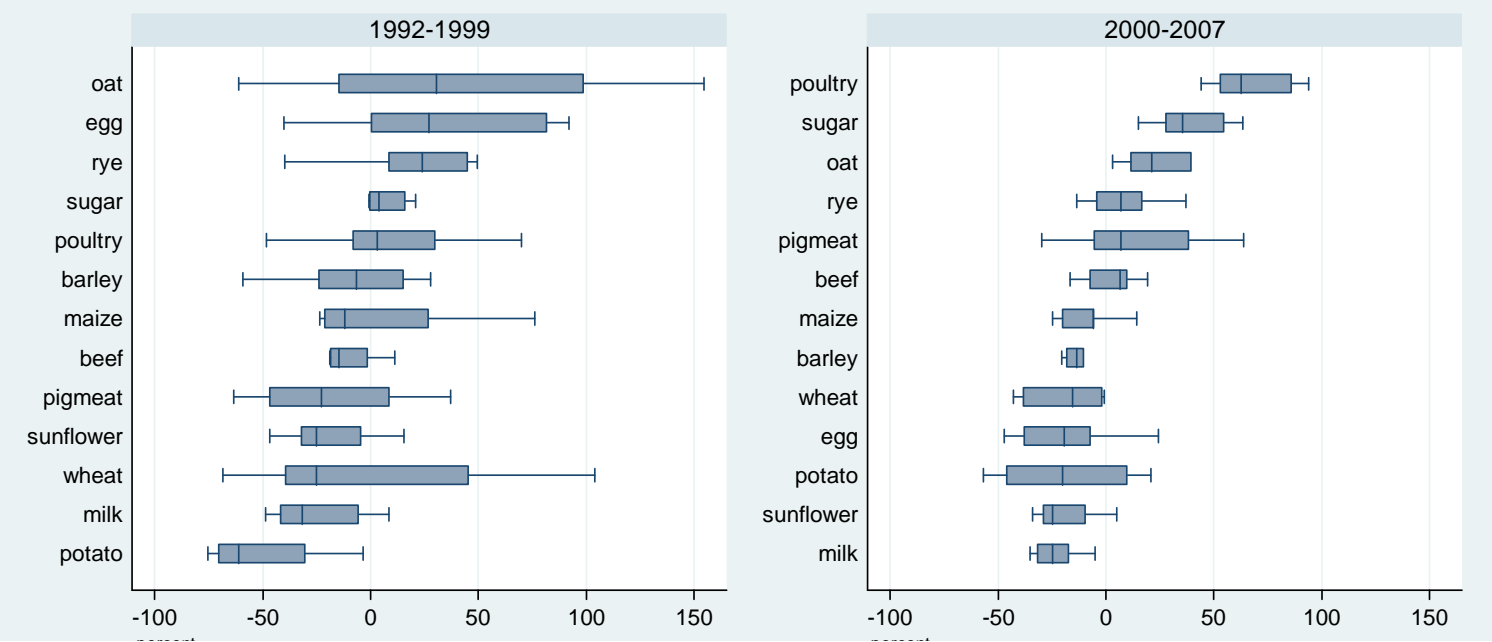
Figure A.4 (continued): Box plot distributions of NRAs of covered agricultural products for each focus country, 1955 to 2007

(e) 9 high-income economies (counting as 1 the EU member countries as of 1/1/2004)

Australia $(\mathrm{n}=1317)$
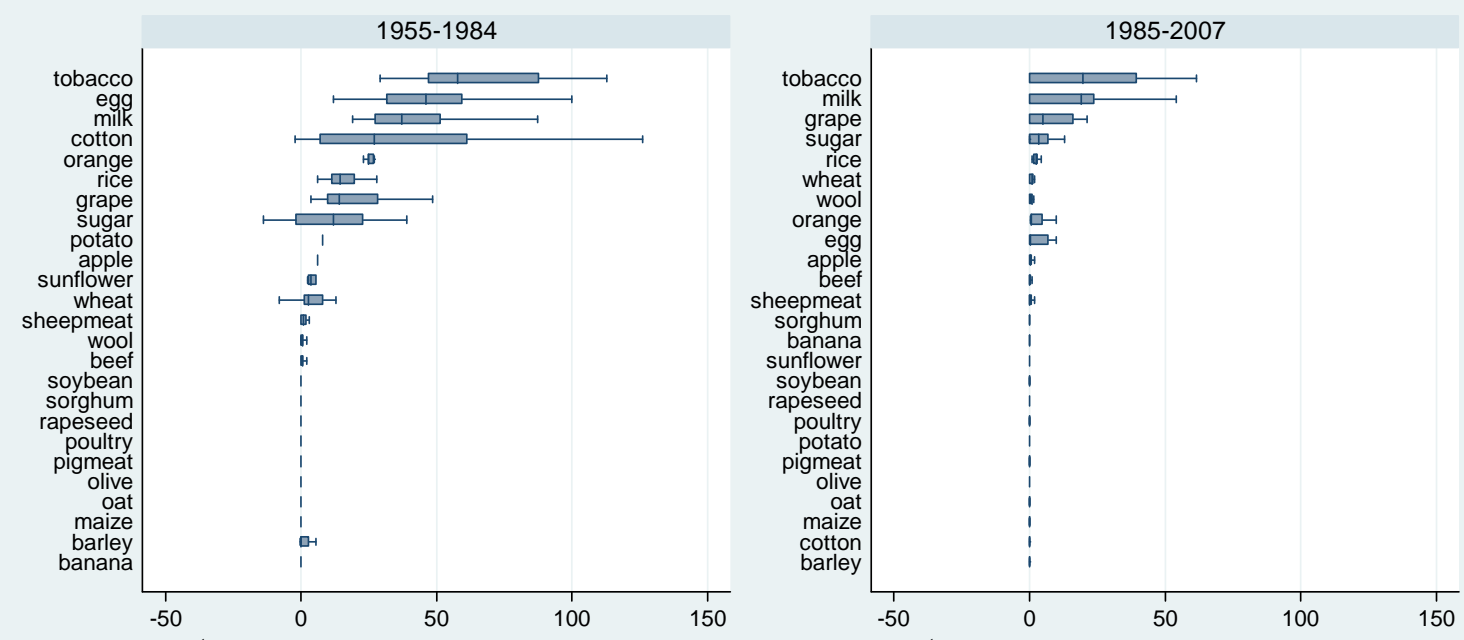

Canada $(\mathrm{n}=630)$
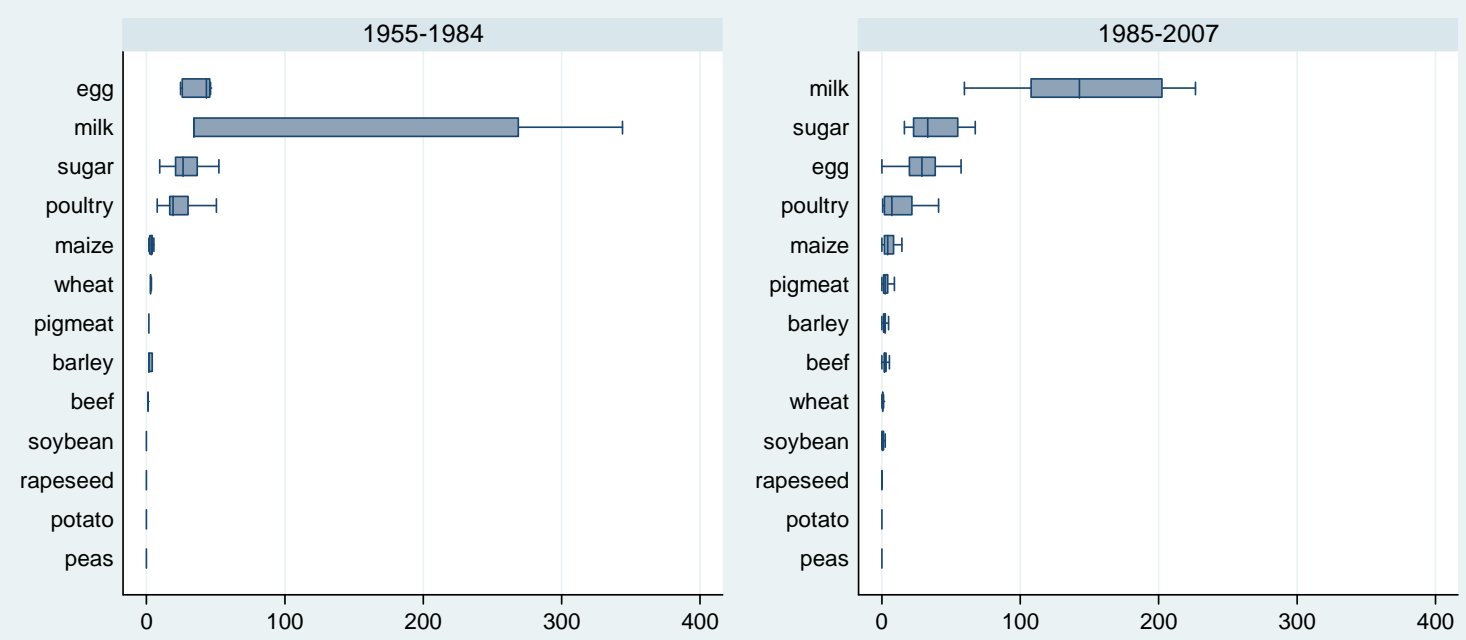

EU-15 countries (as of $1 / 1 / 2004)(n=8157)$
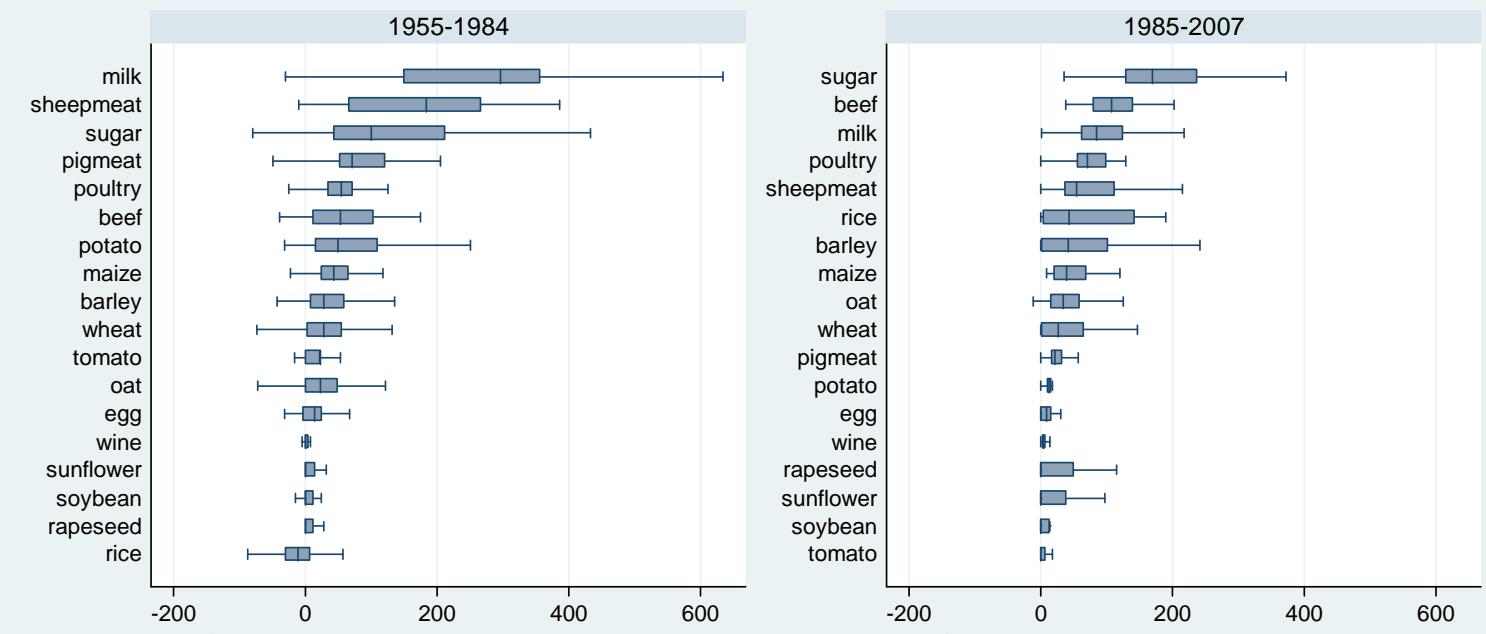
Iceland $(\mathrm{n}=203)$
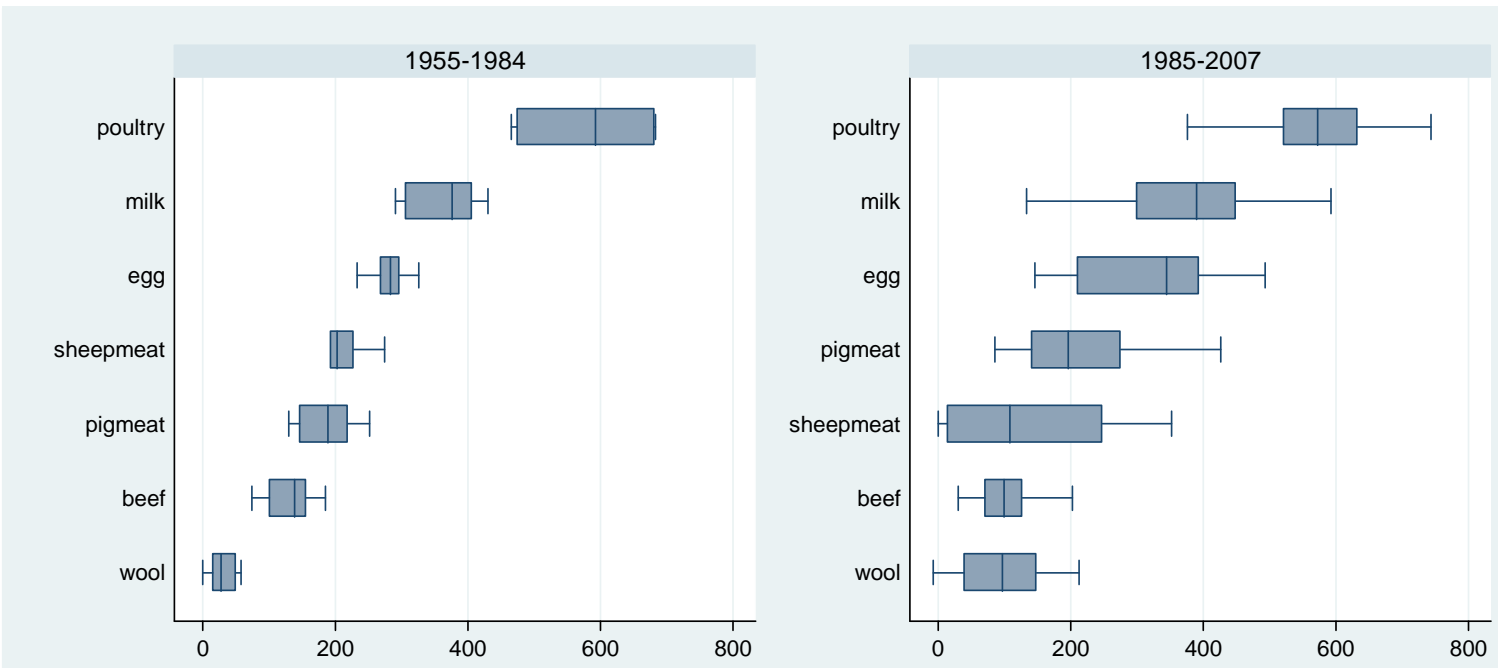

Japan $(\mathrm{n}=1007)$
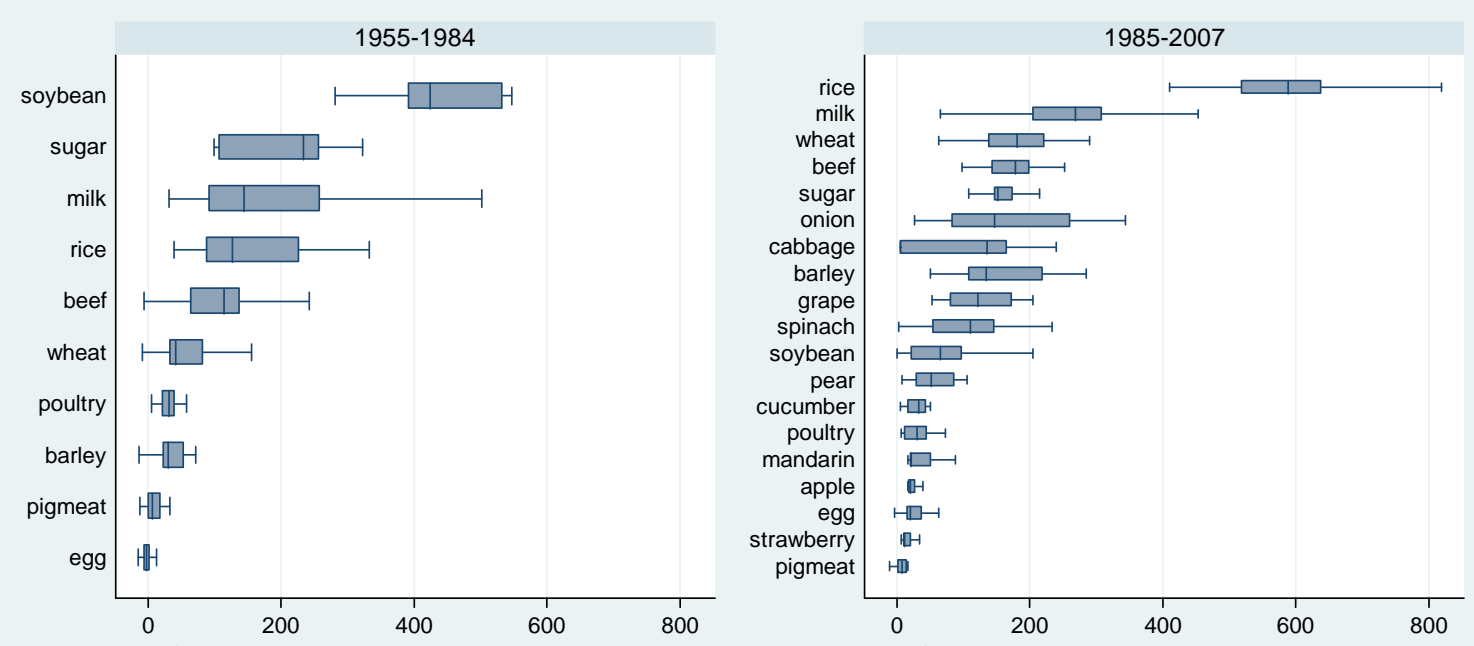

New Zealand $(\mathrm{n}=723)$
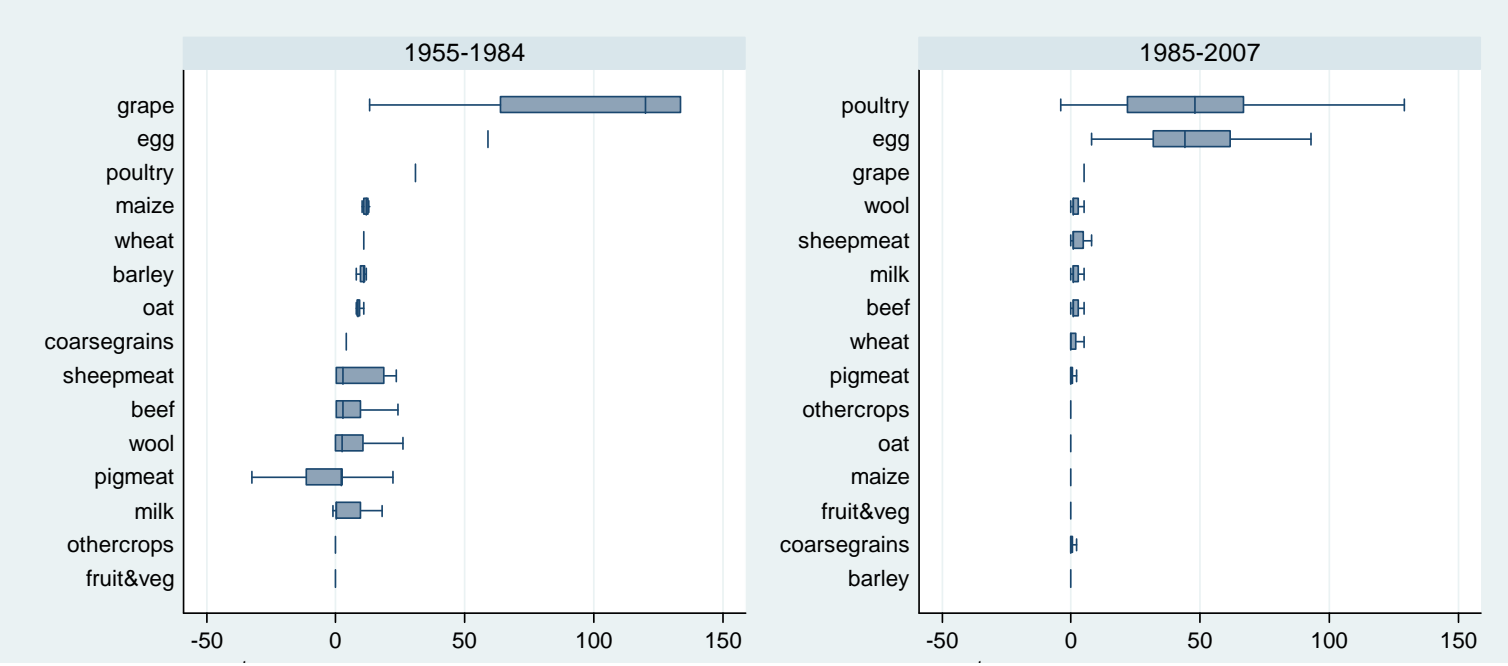
Norway $(n=249)$

Switzerland $(\mathrm{n}=268)$
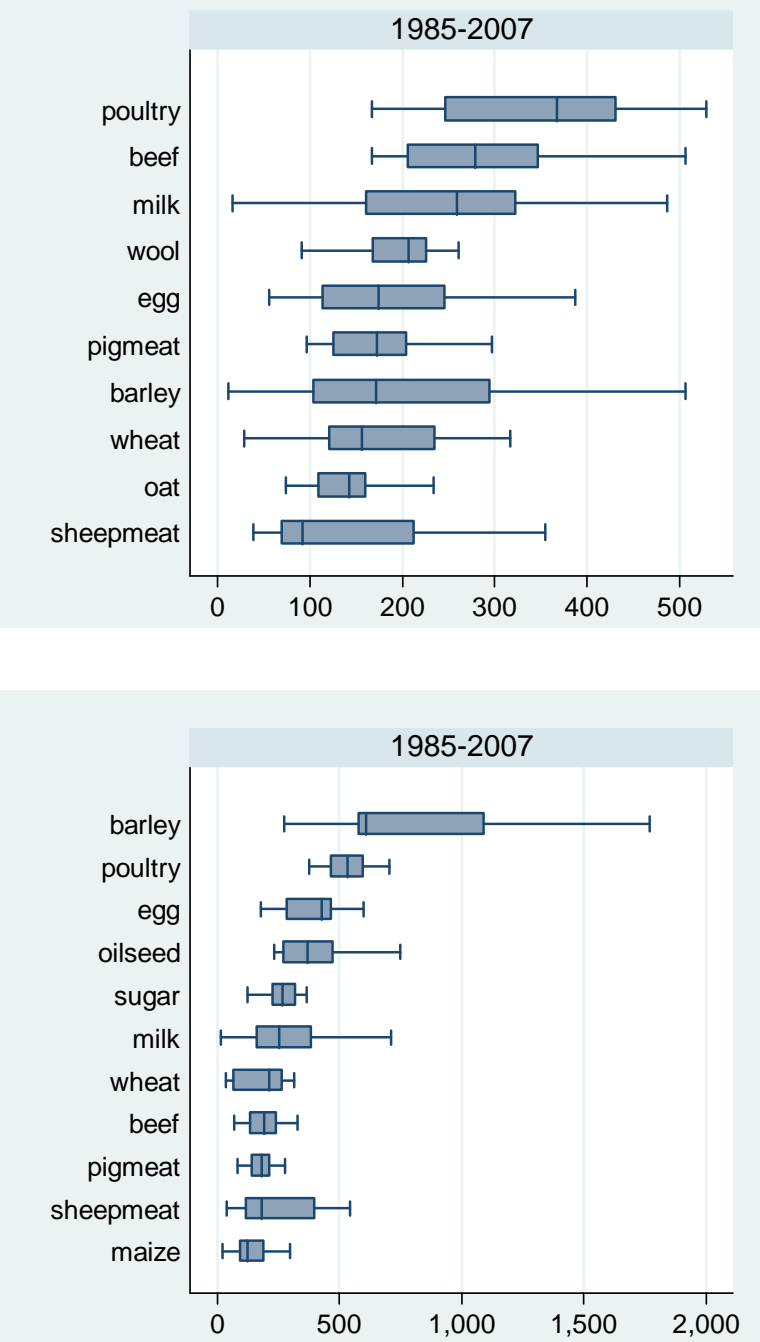

US $(n=721)$
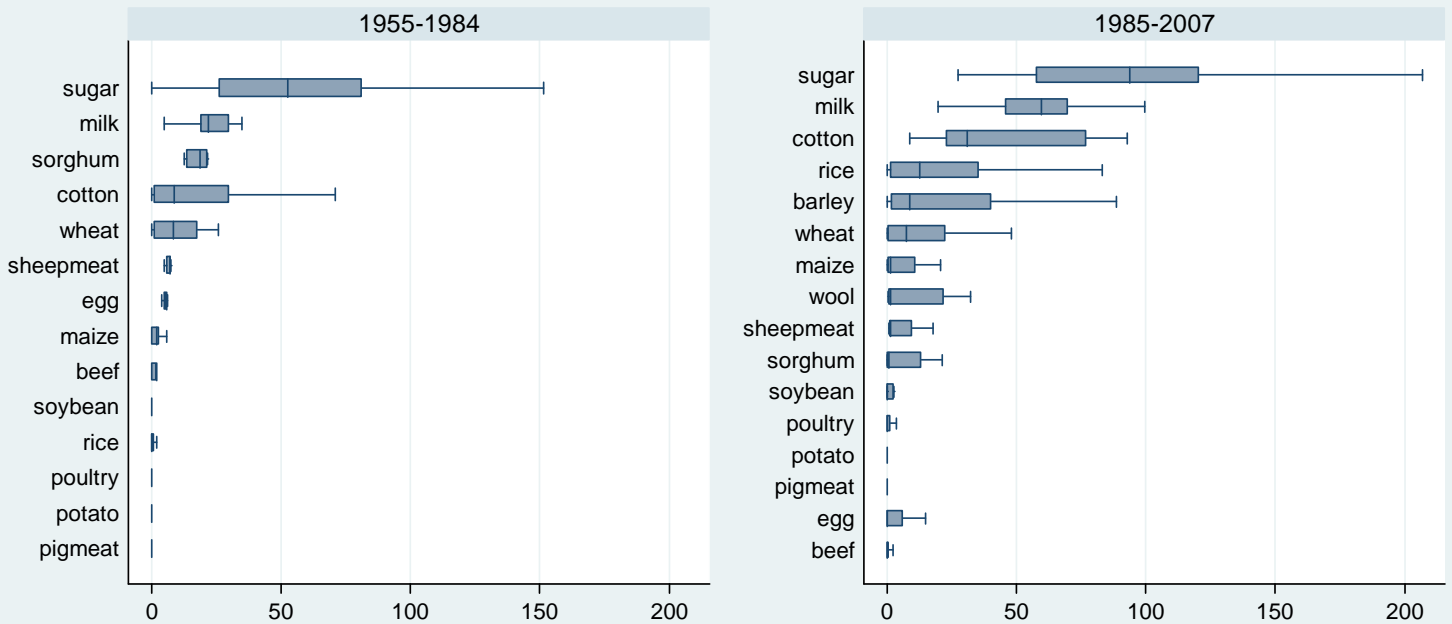

Source: Drawn from estimates in Anderson and Valenzuela (2008). 